\title{
The focus case of a nonsmooth Rayleigh-Duffing oscillator
}

\author{
Zhaoxia Wang ${ }^{1}$, Hebai Chen ${ }^{2}$, Yilei Tang ${ }^{*}$ \\ ${ }^{1}$ School of Mathematical Sciences, University of Electronic Science and Technology of China \\ Chengdu, Sichuan 611731, P. R. China \\ 2 School of Mathematics and Statistics, HNP-LAMA, Central South University \\ Changsha, Hunan 410083, P. R. China \\ 3 School of Mathematical Sciences, MOE-LSC, Shanghai Jiao Tong University \\ Shanghai 200240, P. R. China
}

\begin{abstract}
In this paper, we study the global dynamics of a nonsmooth Rayleigh-Duffing equation $\ddot{x}+a \dot{x}+b \dot{x}|\dot{x}|+c x+d x^{3}=0$ for the case $d>0$, i.e., the focus case. The global dynamics of this nonsmooth Rayleigh-Duffing oscillator for the case $d<0$, i.e., the saddle case, has been studied completely in the companion volume [Int. J. Non-Linear Mech., 129 (2021) 103657]. The research for the focus case is more complex than the saddle case, such as the appearance of five limit cycles and the gluing bifurcation which means that two double limit cycle bifurcation curves and one homoclinic bifurcation curve are very adjacent occurs. We present bifurcation diagram, including one pitchfork bifurcation curve, two Hopf bifurcation curves, two double limit cycle bifurcation curves and one homoclinic bifurcation curve. Finally, numerical phase portraits illustrate our theoretical results.
\end{abstract}

Keywords: Nonsmooth Rayleigh-Duffing oscillator, double limit cycle bifurcation, Hopf bifurcation, homoclinic bifurcation, gluing bifurcation.

AMS (2010) Classification: 34C07, 34C23, 34C $37,34 \mathrm{~K} 18$

\section{Introduction and main results}

In the middle of 17 th century, C. Huygens built the theory of physical pendulum for the first time, and created the single pendulum mechanical clock based on a simple oscillator model. In the study of modern nonlinear science, the dynamics of oscillators plays a more and more important role. A large number of researches on the dynamics of oscillators have emerged; see examples in $[4,5,11,12,15,18]$.

The Rayleigh-Duffing oscillator describes the combination of a nonlinear damping and a nonlinear stiffness $[15,8]$. It can be applied in several mechanical problems, such as the ship roll damping [1], the oscillation of pipes in heat exchangers [10], the planar flow-induced oscillation [16]. A nonsmooth Rayleigh-Duffing equation

$$
\left\{\begin{array}{l}
\dot{x}=y \\
\dot{y}=-c x-d x^{3}-(a+b|y|) y
\end{array}\right.
$$

\footnotetext{
*Email: zxwang@uestc.edu.cn (Z. Wang), chen_hebai@csu.edu.cn (H. Chen, corresponding author), mathtyl@sjtu.edu.cn (Y. Tang)
} 
for $b d \neq 0$ has been researched in $[1,13,20]$ for its dynamics. With the scaling

$$
(x, y, t) \rightarrow\left(\frac{x}{b}, \frac{\sqrt{|d|}}{b|b|}, \frac{|b| t}{\sqrt{|d|}}\right),
$$

system (1.1) can be simplified into

$$
\begin{aligned}
& \text { the saddle case }:\left\{\begin{array}{l}
\dot{x}=y, \\
\dot{y}=\mu_{1} x+x^{3}-\left(\mu_{2}+|y|\right) y,
\end{array}\right. \\
& \text { the focus case }:\left\{\begin{array}{l}
\dot{x}=y, \\
\dot{y}=\mu_{1} x-x^{3}-\left(\mu_{2}+|y|\right) y,
\end{array}\right.
\end{aligned}
$$

where $\mu_{1}$ and $\mu_{2}$ are real. It is clear that the vector fields of systems (1.2a) and (1.2b) are only $C^{1}$. Since both systems (1.2a) and (1.2b) are invariant under the transformation $(x, y) \rightarrow(-x,-y)$, their qualitative structures are symmetric with respect to the origin. So it is sufficient to study their dynamics in the half plane $x \geq 0$. A general introduction for the oscillator model (1.1) which describes some mechanical problems was given in $[1,13,20]$ and the references therein. Moreover, Chen and Wang in [20] made a study for the saddle case of system (1.1), i.e., system (1.2a).

The goal of this paper is to deal with system (1.2b) and present the global bifurcation diagram and all global phase portraits in the Poincaré disc for the focus case. The following two theorems are our main results.

Theorem 1.1. The global bifurcation diagram of system (1.2b) consists of the following bifurcation curves:

(a) pitchfork bifurcation curve $P:=\left\{\left(\mu_{1}, \mu_{2}\right) \in \mathbb{R}^{2}: \mu_{1}=0\right\}$;

(b) generalized Hopf bifurcation curves

$$
H_{1}:=\left\{\left(\mu_{1}, \mu_{2}\right) \in \mathbb{R}^{2}: \mu_{1}>0, \mu_{2}=0\right\} \quad \text { and } H_{2}:=\left\{\left(\mu_{1}, \mu_{2}\right) \in \mathbb{R}^{2}: \mu_{1} \leq 0, \mu_{2}=0\right\}
$$

(c) homoclinic bifurcation curve $H L:=\left\{\left(\mu_{1}, \mu_{2}\right) \in \mathbb{R}^{2}: \mu_{1}>0, \mu_{2}=\varphi\left(\mu_{1}\right)\right\}$;

(d) double large limit cycle bifurcation curves $D L_{1}:=\left\{\left(\mu_{1}, \mu_{2}\right) \in \mathbb{R}^{2}: \mu_{1}>0, \mu_{2}=\varrho_{1}\left(\mu_{1}\right)\right\}$;

(e) double small limit cycle bifurcation curve $D L_{2}:=\left\{\left(\mu_{1}, \mu_{2}\right) \in \mathbb{R}^{2}: \mu_{1}>0, \mu_{2}=\varrho_{2}\left(\mu_{1}\right)\right\}$,

where functions $\varphi\left(\mu_{1}\right), \varrho_{1}\left(\mu_{1}\right)$ and $\varrho_{2}\left(\mu_{1}\right)$ are continuous and decreasing in $\mu_{1}$ for $\mu_{1}>0$, satisfying $-2\left(12 \mu_{1}^{3}\right)^{1 / 4} / 3<\varrho_{2}\left(\mu_{1}\right)<\varphi\left(\mu_{1}\right)<\varrho_{1}\left(\mu_{1}\right)<0$.

Based on the numerical simulations and the results in focus case of smooth Rayleigh-Duffing oscillator [6], we conjecture that the number of small limit cycles only surrounding $\left(\sqrt{\mu_{1}}, 0\right)$ is no more than 2 when $\mu_{1}>0$ and $-2\left(12 \mu_{1}^{3}\right)^{1 / 4} / 3<\mu_{2}<0$. Then, the global bifurcation diagram and global phase portraits in the Poincaré disc are represented in the following theorem. 
Theorem 1.2. The global bifurcation diagram and global phase portraits in the Poincaré disc of (1.2b) are given in Fig. 1, where

$$
\begin{aligned}
\mathrm{I} & :=\left\{\left(\mu_{1}, \mu_{2}\right) \in \mathbb{R}^{2}: \mu_{1}<0, \mu_{2}<0\right\} \\
\mathrm{II} & :=\left\{\left(\mu_{1}, \mu_{2}\right) \in \mathbb{R}^{2}: \mu_{1}<0, \mu_{2}>0\right\}, \\
\mathrm{III} & :=\left\{\left(\mu_{1}, \mu_{2}\right) \in \mathbb{R}^{2}: \mu_{1}>0, \mu_{2}>0\right\}, \\
\mathrm{IV} & :=\left\{\left(\mu_{1}, \mu_{2}\right) \in \mathbb{R}^{2}: \mu_{1}>0, \varrho_{1}\left(\mu_{1}\right)<\mu_{2}<0\right\}, \\
\mathrm{V} & :=\left\{\left(\mu_{1}, \mu_{2}\right) \in \mathbb{R}^{2}: \mu_{1}>0, \varphi\left(\mu_{1}\right)<\mu_{2}<\varrho_{1}\left(\mu_{1}\right)\right\}, \\
\mathrm{VI} & :=\left\{\left(\mu_{1}, \mu_{2}\right) \in \mathbb{R}^{2}: \mu_{1}>0, \varrho_{2}\left(\mu_{1}\right)<\mu_{2}<\varphi\left(\mu_{1}\right\},\right. \\
\mathrm{VII} & :=\left\{\left(\mu_{1}, \mu_{2}\right) \in \mathbb{R}^{2}: \mu_{1}>0, \mu_{2}<\varrho_{2}\left(\mu_{1}\right)\right\} \\
P_{1} & :=\left\{\left(\mu_{1}, \mu_{2}\right) \in \mathbb{R}^{2}: \mu_{1}=0, \mu_{2}<0\right\}, \\
P_{2} & :=\left\{\left(\mu_{1}, \mu_{2}\right) \in \mathbb{R}^{2}: \mu_{1}=0, \mu_{2} \geq 0\right\} .
\end{aligned}
$$

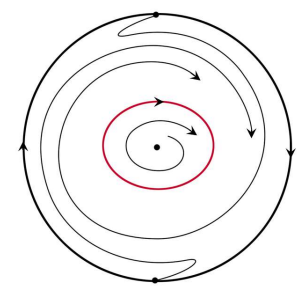

I $\cup P_{1}$

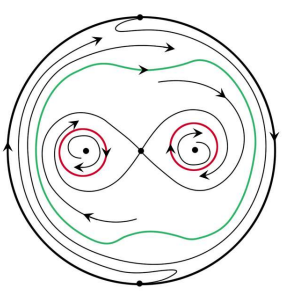

$D L_{1}$

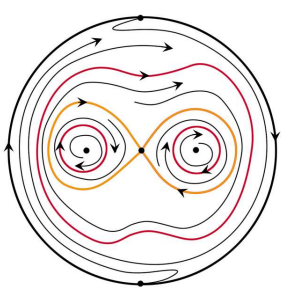

$H L$

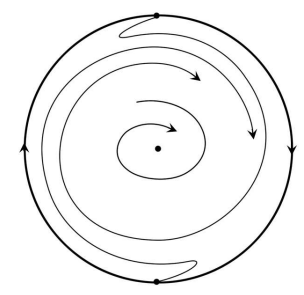

II $\cup \mathrm{P}_{2} \cup \mathrm{H}_{2}$

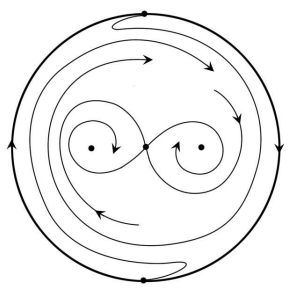

III $\cup H_{1}$
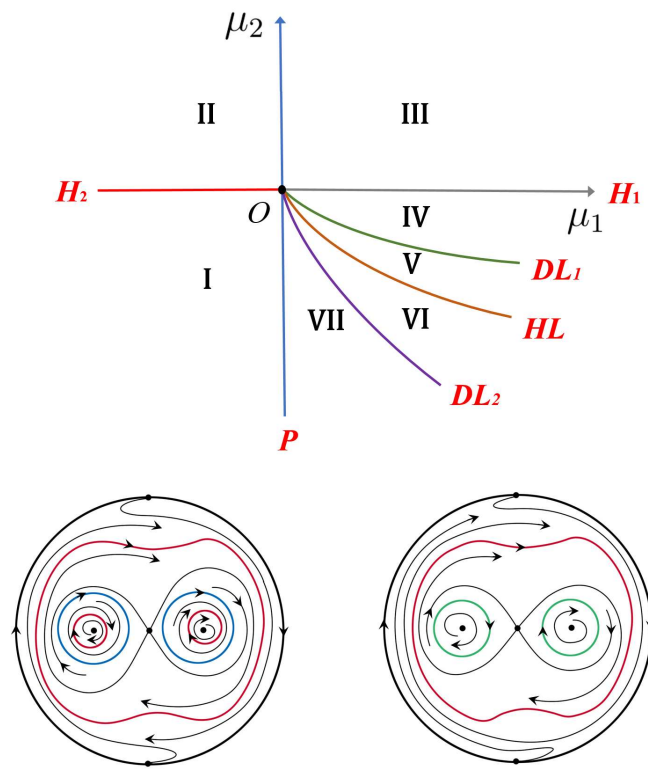

VI

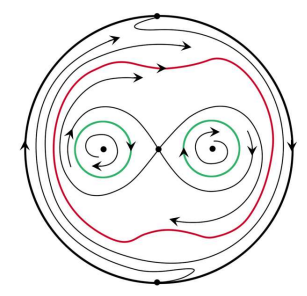

$D L_{2}$

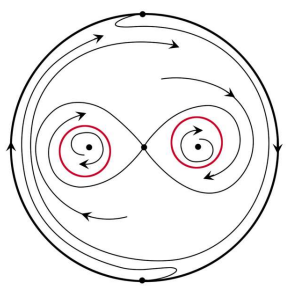

IV
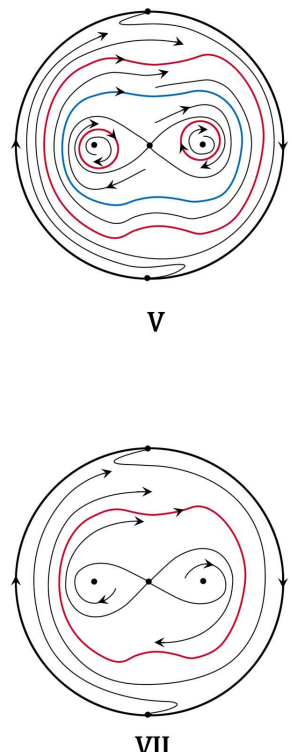

VII

Fig. 1: The bifurcation diagram and corresponding global phase portraits.

Notice that the stable manifold on the right-hand side of the saddle at the origin in III $\cup H_{1}$ or IV possibly connects with equilibria at infinity from either the positive $y$-axis or the negative 
y-axis, as shown in Fig. 2. For simplicity, we only present one of these possibilities in Fig. 1.

In addition, three bifurcation curves $D L_{1}, D L_{2}$ and $H L$ are very close, implying the appearance of the gluing bifurcation. We give some numerical simulations to show this bifurcation phenomenon in Section 5.

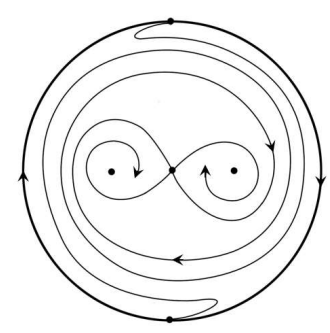

$\left(\mathrm{III} \cup H_{1}\right)-1$

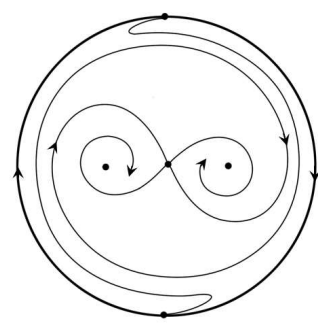

(III $\left.\cup H_{1}\right)-2$

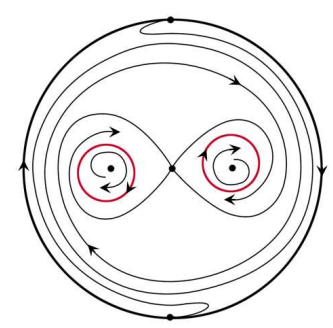

IV-1

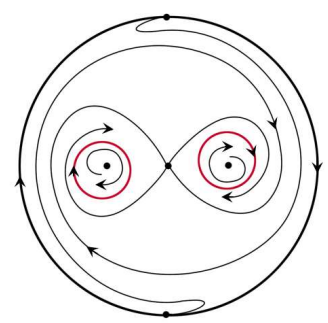

IV-2

Fig. 2: Possibilities of orbit connections at infinity for $\left(\mu_{1}, \mu_{2}\right) \in \mathrm{III} \cup H_{1}$ or $\left(\mu_{1}, \mu_{2}\right) \in \mathrm{IV}$.

An outline of this paper is as follows. Local dynamics of system (1.2b) are studied in Section 2 , such as qualitative properties of equilibria (including equilibria at infinity) of system (1.2b) and local bifurcations. In Section 3, we study the limit cycles and homoclinic loops of system (1.2b). Theorems 1.1 and 1.2 are proven in Section 4. In Section 5, numerical simulations illustrate our analytical results. In Section 6, we conclude the global dynamics for the focus cases of smooth and nonsmooth Rayleigh-Duffing oscillators and van der Pol-Duffing oscillators.

\section{Local dynamics of system (1.2b)}

It is easy to obtain from solving equations $\dot{x}=\dot{y}=0$ for system (1.2b) that there exist three equilibria $E_{0}:(0,0), E_{l}:\left(-\sqrt{\mu_{1}}, 0\right), E_{r}:\left(\sqrt{\mu_{1}}, 0\right)$ when $\mu_{1}>0$ and a unique equilibrium $E_{0}:(0,0)$ when $\mu_{1} \leq 0$, respectively. We give the qualitative properties of equilibria of system $(1.2 \mathrm{~b})$ in the following lemmas.

Lemma 2.1. When $\mu_{1}>0$, there are three equilibria $E_{0}:(0,0), E_{l}:\left(-\sqrt{\mu_{1}}, 0\right), E_{r}:\left(\sqrt{\mu_{1}}, 0\right)$ for system (1.2b) and their qualitative properties are shown in Table 1.

\begin{tabular}{|l|l|}
\hline conditions of parameters & qualitative properties of equilibria \\
\hline$\mu_{1}>0, \mu_{2}>2 \sqrt{2 \mu_{1}}$ & $E_{0}$ saddle; $E_{l}, E_{r}$ stable bidirectional nodes \\
\hline$\mu_{1}>0, \mu_{2}=2 \sqrt{2 \mu_{1}}$ & $E_{0}$ saddle; $E_{l}, E_{r}$ stable unidirectional nodes \\
\hline$\mu_{1}>0,0<\mu_{2}<2 \sqrt{2 \mu_{1}}$ & $E_{0}$ saddle; $E_{l}, E_{r}$ stable rough foci \\
\hline$\mu_{1}>0, \mu_{2}=0$ & $E_{0}$ saddle; $E_{l}, E_{r}$ stable weak foci \\
\hline$\mu_{1}>0,-2 \sqrt{2 \mu_{1}}<\mu_{2}<0$ & $E_{0}$ saddle; $E_{l}, E_{r}$ unstable rough foci \\
\hline$\mu_{1}>0, \mu_{2}=-2 \sqrt{2 \mu_{1}}$ & $E_{0}$ saddle; $E_{l}, E_{r}$ unstable unidirectional nodes \\
\hline$\mu_{1}>0, \mu_{2}<-2 \sqrt{2 \mu_{1}}$ & $E_{0}$ saddle; $E_{l}, E_{r}$ unstable bidirectional nodes \\
\hline
\end{tabular}

Table 1: Properties of $E_{0}, E_{l}$ and $E_{r}$ when $\mu_{1}>0$. 
Proof. Notice that the Jacobian matrix for system (1.2b) at $E_{0}$ is

$$
J_{0}=\left(\begin{array}{cc}
0 & 1 \\
\mu_{1} & -\mu_{2}
\end{array}\right)
$$

and $\operatorname{det} J_{0}=-\mu_{1}<0$. Since the nonlinear terms in system $(1.2 \mathrm{~b})$ at $E_{0}$ are continuously differentiable, $E_{0}$ is a saddle by [21, Theorem 4.4 of Chapter 4$]$.

The Jacobian matrices for system $(1.2 \mathrm{~b})$ at $E_{l}$ and $E_{r}$ are both equal to

$$
J_{l}=J_{r}=\left(\begin{array}{cc}
0 & 1 \\
-2 \mu_{1} & -\mu_{2}
\end{array}\right) \text {. }
$$

It follows that $\operatorname{det} J_{l}=\operatorname{det} J_{r}=2 \mu_{1}>0$ and $\operatorname{tr} J_{l}=\operatorname{tr} J_{r}=-\mu_{2}$. Then $\Delta=\mu_{2}^{2}-8 \mu_{1}$. Thus, $E_{l}$ and $E_{r}$ are stable (resp. unstable) bidirectional nodes if $\mu_{2}>2 \sqrt{2 \mu_{1}}$ (resp. $\mu_{2}<-2 \sqrt{2 \mu_{1}}$ ), stable (resp. unstable) unidirectional nodes if $\mu_{2}=2 \sqrt{2 \mu_{1}}$ (resp. $\mu_{2}=-2 \sqrt{2 \mu_{1}}$ ), stable (resp. unstable) rough foci if $0<\mu_{2}<2 \sqrt{2 \mu_{1}}$ (resp. $-2 \sqrt{2 \mu_{1}}<\mu_{2}<0$ ).

Consider the case $\mu_{2}=0$. It is sufficient to study the qualitative properties of $E_{r}$ because of the symmetry of the system. By the transformation $(x, y) \rightarrow\left(x+\sqrt{\mu_{1}}, y\right)$, which translates the equilibrium $E_{r}$ to the origin, system $(1.2 \mathrm{~b})$ can be reduced into

$$
\dot{x}=y, \quad \dot{y}=-2 \mu_{1} x-3 \sqrt{\mu_{1}} x^{2}-|y| y-x^{3} .
$$

Applying the transformation

$$
(x, y, t) \rightarrow\left(x /\left(2 \mu_{1}\right), y / \sqrt{2 \mu_{1}},-t / \sqrt{2 \mu_{1}}\right),
$$

system (2.2) can be written as

$$
\dot{x}=-y, \quad \dot{y}=x+\frac{3 x^{2}}{4 \mu_{1} \sqrt{\mu_{1}}}+\frac{|y| y}{2 \mu_{1}}+\frac{x^{3}}{8 \mu_{1}^{3}} .
$$

In order to calculate focal values for nonsmooth system (2.3) we take polar coordinates $x=r \cos \theta$ and $y=r \sin \theta$ to translate (2.3) into

$$
\frac{d r}{d \theta}=\frac{\left(3 \sin \theta \cos ^{2} \theta /\left(4 \mu_{1} \sqrt{\mu_{1}}\right)+|\sin \theta| \sin ^{2} \theta /\left(2 \mu_{1}\right)\right) r^{2}+\sin \theta \cos ^{3} \theta r^{3} /\left(8 \mu_{1}^{3}\right)}{1+\left(3 \cos ^{3} \theta /\left(4 \mu_{1} \sqrt{\mu_{1}}\right)+|\sin \theta| \sin \theta \cos \theta /\left(2 \mu_{1}\right)\right) r+\cos ^{4} \theta r^{2} /\left(8 \mu_{1}^{3}\right)} .
$$

Consider the solution $r\left(\theta, r_{0}\right)$ of $(2.4)$ with the initial condition $r\left(0, r_{0}\right)=r_{0}$, where $r_{0}>0$ is sufficient small. Obviously, $r\left(\theta, r_{0}\right)$ can be written as $r^{+}\left(\theta, r_{0}\right)=r_{0}+\sum_{i=2}^{\infty} r_{i}^{+}(\theta) r_{0}^{i}$ when $0 \leq \theta \leq \pi$ and $r^{-}\left(\theta, r_{0}\right)=r_{0}+\sum_{i=2}^{\infty} r_{i}^{-}(\theta) r_{0}^{i}$ when $\pi \leq \theta \leq 2 \pi$. Substituting them into (2.4), one can obtain

$$
r^{+}\left(\pi, r_{0}\right)-r_{0}=\frac{4 \sqrt{\mu_{1}}+3}{6 \mu_{1} \sqrt{\mu_{1}}} r_{0}^{2}+O\left(r_{0}^{3}\right)
$$

and

$$
r^{-}\left(-\pi, r_{0}\right)-r_{0}=\frac{-4 \sqrt{\mu_{1}}+3}{6 \mu_{1} \sqrt{\mu_{1}}} r_{0}^{2}+O\left(r_{0}^{3}\right) .
$$

Then

$$
r^{+}\left(\pi, r_{0}\right)-r^{-}\left(-\pi, r_{0}\right)=\frac{4}{3 \mu_{1}} r_{0}^{2}+O\left(r_{0}^{3}\right)>0,
$$

which implies that the origin is an unstable weak focus of system (2.3) and then $E_{r}$ is a stable weak focus when $\mu_{2}=0$. So is $E_{l}$. 
Lemma 2.2. When $\mu_{1} \leq 0$, there is a unique equilibrium $E_{0}:(0,0)$ for system $(1.2 \mathrm{~b})$. Moreover, the properties of $E_{0}$ are shown in Table 2.

\begin{tabular}{|c|l|l|}
\hline \multicolumn{2}{|c|}{ conditions of parameters } & qualitative properties of equilibria \\
\hline \multirow{4}{*}{$\mu_{1}=0$} & $\mu_{2}>0$ & $E_{0}$ stable degenerate node \\
\cline { 2 - 3 } & $\mu_{2}=0$ & $E_{0}$ stable nilpotent focus \\
\cline { 2 - 3 } & $\mu_{2}<0$ & $E_{0}$ unstable degenerate node \\
\hline \multirow{4}{*}{$\mu_{1}<0$} & $\mu_{2}>2 \sqrt{-\mu_{1}}$ & $E_{0}$ stable bidirectional node \\
\cline { 2 - 3 } & $\mu_{2}=2 \sqrt{-\mu_{1}}$ & $E_{0}$ stable unidirectional node \\
\cline { 2 - 3 } & $0<\mu_{2}<2 \sqrt{-\mu_{1}}$ & $E_{0}$ stable rough focus \\
\cline { 2 - 3 } & $\mu_{2}=0$ & $E_{0}$ stable weak focus \\
\cline { 2 - 3 } & $-2 \sqrt{-\mu_{1}}<\mu_{2}<0$ & $E_{0}$ unstable rough focus \\
\cline { 2 - 3 } & $\mu_{2}=-2 \sqrt{-\mu_{1}}$ & $E_{0}$ unstable unidirectional node \\
\cline { 2 - 3 } & $\mu_{2}<-2 \sqrt{-\mu_{1}}$ & $E_{0}$ unstable bidirectional node \\
\hline
\end{tabular}

Table 2: Properties of $E_{0}, E_{L}$ and $E_{R}$ when $\mu_{1} \leq 0$.

Proof. The Jacobian matrix for system $(1.2 \mathrm{~b})$ at $E_{0}$ is still (2.1), which implies that $\operatorname{det} J_{0}=-\mu_{1}$ and $\operatorname{tr} J_{0}=-\mu_{2}$. Moreover, $\Delta=\mu_{2}^{2}+4 \mu_{1}$. When $\mu_{1}<0$ and $\mu_{2} \neq 0, E_{0}$ is a stable (resp. unstable) bidirectional node if $\mu_{2}>2 \sqrt{-\mu_{1}}$ (resp. $\mu_{2}<-2 \sqrt{-\mu_{1}}$ ), a stable (resp. unstable) unidirectional node if $\mu_{2}=2 \sqrt{-\mu_{1}}$ (resp. $\mu_{2}=-2 \sqrt{-\mu_{1}}$ ), a stable (resp. unstable) rough focus if $0<\mu_{2}<2 \sqrt{-\mu_{1}}$ (resp. $\left.-2 \sqrt{-\mu_{1}}<\mu_{2}<0\right)$.

When $\mu_{1}<0$ and $\mu_{2}=0$, by the transformation

$$
(x, y, t) \rightarrow\left(-x / \mu_{1}, y / \sqrt{-\mu_{1}},-t / \sqrt{-\mu_{1}}\right),
$$

system $(1.2 \mathrm{~b})$ can be reduced into

$$
\dot{x}=-y, \quad \dot{y}=x-\frac{|y| y}{\mu_{1}}-\frac{x^{3}}{\mu_{1}^{3}} .
$$

Taking polar coordinates $x=r \cos \theta$ and $y=r \sin \theta$, we have

$$
\frac{d r}{d \theta}=\frac{-\sin \theta \cos ^{3} \theta r^{3} / \mu_{1}^{3}-|\sin \theta| \sin ^{2} \theta r^{2} / \mu_{1}}{1-\cos ^{4} \theta r^{2} / \mu_{1}^{3}-|\sin \theta| \sin \theta \cos \theta r / \mu_{1}} .
$$

Similarly, the solution $r\left(\theta, r_{0}\right)$ of $(2.6)$ with the initial condition $r\left(0, r_{0}\right)=r_{0}$ can be written as $r^{+}\left(\theta, r_{0}\right)=r_{0}+\sum_{i=2}^{\infty} r_{i}^{+}(\theta) r_{0}^{i}$ when $0 \leq \theta \leq \pi$ and $r^{-}\left(\theta, r_{0}\right)=r_{0}+\sum_{i=2}^{\infty} r_{i}^{-}(\theta) r_{0}^{i}$ when $-\pi \leq \theta \leq 0$, where $r_{0}>0$ is sufficiently small. Substituting them into (2.6), one can obtain that

$$
r^{+}\left(\pi, r_{0}\right)-r_{0}=-\frac{4}{3 \mu_{1}} r_{0}^{2}+O\left(r_{0}^{3}\right)
$$

and

$$
r^{-}\left(-\pi, r_{0}\right)-r_{0}=\frac{4}{3 \mu_{1}} r_{0}^{2}+O\left(r_{0}^{3}\right)
$$

Then

$$
r^{+}\left(\pi, r_{0}\right)-r^{-}\left(-\pi, r_{0}\right)=-\frac{8}{3 \mu_{1}} r_{0}^{2}+O\left(r_{0}^{3}\right)>0
$$


which implies that the origin of system (2.5) is an unstable weak focus and then $E_{0}$ is a stable weak focus when $\mu_{1}<0$ and $\mu_{2}=0$.

When $\mu_{1}=0$ and $\mu_{2} \neq 0$, the Jacobian matrix (2.1) only has one nonzero eigenvalue. We consider exceptional directions of system (1.2b) instead of blow-up method to get qualitative properties of $E_{0}$, because system (1.2b) is not analytic at $E_{0}$. With polar coordinates $x=r \cos \theta$ and $y=r \sin \theta$, system $(1.2 \mathrm{~b})$ is changed into

$$
\frac{1}{r} \frac{d r}{d \theta}=\frac{H(\theta)+O(r)}{G(\theta)+O(r)}, \quad \text { as } \quad r \rightarrow 0,
$$

where

$$
G(\theta)=-\sin ^{2} \theta-\mu_{2} \sin \theta \cos \theta \text { and } H(\theta)=-\mu_{2} \sin ^{2} \theta+\sin \theta \cos \theta .
$$

Then the zeros of $G(\theta)$ on the interval $[0,2 \pi)$ depend on the sign of $\mu_{2}$.

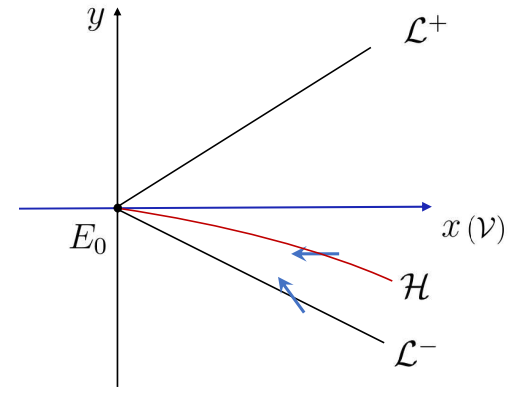

(a) $\mu_{1}=0, \mu_{2}>0$

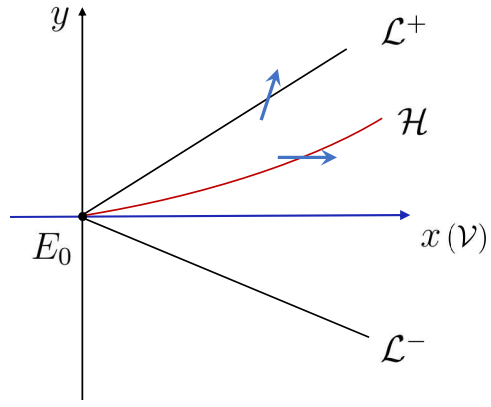

(b) $\mu_{1}=0, \mu_{2}<0$

Fig. 3: Dynamical behaviors near $E_{0}$.

When $\mu_{1}=0$ and $\mu_{2}>0$, the equation $G(\theta)=0$ has exactly four real roots $0, \pi-\arctan \left(\mu_{2}\right)$, $\pi$ and $2 \pi-\arctan \left(\mu_{2}\right)$ in the interval $[0,2 \pi)$. It is enough to investigate two directions $\theta=0$ and $\theta=\pi-\arctan \left(\mu_{2}\right)$ because of the symmetry of the system. On the one hand, $\theta=\pi-\arctan \left(\mu_{2}\right)$ is a simple root and

$$
G^{\prime}\left(\pi-\arctan \left(\mu_{2}\right)\right) H\left(\pi-\arctan \left(\mu_{2}\right)\right)=-\mu_{2}^{2}<0, \quad H\left(\pi-\arctan \left(\mu_{2}\right)\right)=-\mu_{2}<0 .
$$

By [21, Theorem 3.7 of Chapter 2], system (1.2b) has a unique orbit approaching $E_{0}$ in the direction $\theta=\pi-\arctan \left(\mu_{2}\right)$ as $t \rightarrow \infty$. On the other hand, $G(0)=H(0)=0$ implies that the method of normal sectors loses effectiveness. Thus, generalized normal sectors (see [17]) will be constructed. From equations in (1.2b) with $\mu_{1}=0$ and $\mu_{2}>0$, it is easy to see that there is a unique vertical isocline

$$
\mathcal{V}:=\left\{(x, y) \in \mathbb{R}^{2}: y=0, \quad 0<x<\ell\right\}
$$

and a unique horizontal isocline

$$
\mathcal{H}:=\left\{(x, y) \in \mathbb{R}^{2}: y=-\frac{x^{3}}{\mu_{2}}+O\left(x^{6}\right), \quad 0<x<\ell\right\},
$$

where $\ell>0$ is sufficiently small. Moreover, let

$$
\mathcal{L}^{+}:=\left\{(x, y) \in \mathbb{R}^{2}: y=x \tan \alpha, \quad 0<x<\ell\right\}
$$


and

$$
\mathcal{L}^{-}:=\left\{(x, y) \in \mathbb{R}^{2}: y=-x \tan \alpha, \quad 0<x<\ell\right\},
$$

where $0<\alpha<\pi / 2$ is chosen to be arbitrarily close to 0 . Obviously, $\mathcal{H}$ is in the fourth quadrant and above $\mathcal{L}^{-}$, as shown in Fig. 3 (a). One can check that $\dot{x}>0$ and $\dot{y}<0$ in the open sub-region $\Delta \widehat{\mathcal{L}^{+} E_{0} \mathcal{V}}$. By [17, Lemma 4], there are no orbits connecting $E_{0}$ in $\Delta \widehat{\mathcal{L}^{+} E_{0} \mathcal{V}}$. Notice that $\dot{x}<0$ and $\dot{y}<0$ in the open region $\Delta \widehat{\mathcal{V E} E_{0} \mathcal{H}}$. There are also no orbits connecting $E_{0}$ in $\Delta \widehat{\mathcal{V E} E_{0} \mathcal{H}}$, because any orbit connecting $E_{0}$ in the second quadrant has a negative slope. Since $\dot{x}<0, \dot{y}>0$ in the open sub-region $\Delta \widehat{\mathcal{H E} \mathcal{L}^{-}}$and

$$
\left.\dot{x}\right|_{\mathcal{H}}=-\frac{x^{3}}{\mu_{2}}+O\left(x^{6}\right)<0,\left.\quad \frac{\dot{y}}{\dot{x}}\right|_{\mathcal{L}^{-}}=-\mu_{2}-x \tan \alpha+x^{2} \cot \alpha<-\tan \alpha,
$$

$\Delta \widehat{\mathcal{H} E_{0} \mathcal{L}^{-}}$is a generalized normal sector of Class I, as shown in Fig. 3 (a). Thus, by [17, Lemma 1] system $(1.2 \mathrm{~b})$ has infinitely many orbits approaching $E_{0}$ in $\Delta \widehat{\mathcal{H} E_{0} \mathcal{L}^{-}}$as $t \rightarrow \infty$. By the symmetry $E_{0}$ is a stable node when $\mu_{1}=0$ and $\mu_{2}>0$.

When $\mu_{1}=0$ and $\mu_{2}<0$, the roots of $G(\theta)=0$ on the interval $[0,2 \pi)$ are $0,-\arctan \left(\mu_{2}\right), \pi$ and $\pi-\arctan \left(\mu_{2}\right)$. Similarly, we only consider $\theta=0$ and $\theta=-\arctan \left(\mu_{2}\right)$, because system $(1.2 \mathrm{~b})$ is symmetric about $E_{0}$. One can check that $\theta=-\arctan \left(\mu_{2}\right)$ is a simple root and

$$
G^{\prime}\left(-\arctan \left(\mu_{2}\right)\right) H\left(-\arctan \left(\mu_{2}\right)\right)=-\mu_{2}^{2}<0, \quad H\left(-\arctan \left(\mu_{2}\right)\right)=-\mu_{2}>0 .
$$

By [21, Theorem 3.7 of Chapter 2], system (1.2b) has a unique orbit leaving $E_{0}$ in the direction $\theta=-\arctan \left(\mu_{2}\right)$ as $t \rightarrow \infty$. Since $G(0)=H(0)=0$, we also need to construct generalized normal sectors near $E_{0}$. The small arcs $\mathcal{V}, \mathcal{H}, \mathcal{L}^{+}$and $\mathcal{L}^{-}$are defined as in the case $\mu_{1}=0$ and $\mu_{2}>0$. But $\mathcal{H}$ is in the first quadrant and below $\mathcal{L}^{+}$because $\mu_{2}<0$, as shown in Fig. 3 (b). Since $\dot{x}>0$, $\dot{y}>0$ in $\widehat{\Delta \mathcal{L}+E_{0} \mathcal{H}}$ and

$$
\left.\dot{x}\right|_{\mathcal{H}}=-\frac{x^{3}}{\mu_{2}}+O\left(x^{6}\right)>0,\left.\quad \frac{\dot{y}}{\dot{x}}\right|_{\mathcal{L}^{-}}=-\mu_{2}-x \tan \alpha-x^{2} \cot \alpha>\tan \alpha,
$$

$\Delta \widehat{\mathcal{L}^{+} E_{0} \mathcal{H}}$ is a generalized normal sector of Class I, as shown in Fig. 3 (b). Thus, system (1.2b) has infinitely many orbits leaving $E_{0}$ in $\widehat{\Delta \mathcal{L}^{+} E_{0} \mathcal{H}}$ as $t \rightarrow \infty$. One can check that $\dot{x}>0$ and $\dot{y}<0$ in $\Delta \widehat{\mathcal{H} E_{0} \mathcal{V}}$. By $\left[17\right.$, Lemma 4], there are no orbits connecting $E_{0}$ in $\Delta \widehat{\mathcal{H} E_{0} \mathcal{V}}$. Since $\dot{x}<0$ and $\dot{y}<0$ in $\Delta \widehat{\mathcal{V E} \mathcal{L}_{0}}$, there are no orbits connecting $E_{0}$ in $\Delta \widehat{\mathcal{V} E_{0} \mathcal{L}^{-}}$. Furthermore, by the symmetry $E_{0}$ is an unstable node when $\mu_{1}=0$ and $\mu_{2}<0$.

When $\mu_{1}=0$ and $\mu_{2}=0$, matrix $J_{0}$ is nilpotent. Notice that system (1.2b) can be written as Bernoulli equation

$$
\frac{d y}{d x}=-|y|-\frac{x^{3}}{y},
$$

which has a general solution $4 x^{3} \operatorname{sgn}(y)-6 x^{2}+6 x \operatorname{sgn}(y)-3-C e^{-2 x \operatorname{sgn}(y)}+4 y^{2}=0$ and $C$ is an arbitrary constant. Then with the initial condition $y(0)=0$, the constant $C$ is determined to be -3 and

$$
4 x^{3} \operatorname{sgn}(y)-6 x^{2}+6 x \operatorname{sgn}(y)-3+3 e^{-2 x \operatorname{sgn}(y)}+4 y^{2}=2 x^{4}+4 y^{2}+O\left(x^{5}\right),
$$


implying that there are no orbits connecting $E_{0}$. Thus, $E_{0}$ is a center or a focus of system $(1.2 \mathrm{~b})$. Assume that $\widehat{A B}$ is the upper half part of an orbit near $E_{0}$, and $A$ (resp. $B$ ) is its intersection point with the negative (resp. positive) $x$-axis. Let $\mathcal{E}_{0}(x, y)=x^{4} / 4+y^{2} / 2$. Then

$$
\mathcal{E}_{0}(B)-\mathcal{E}_{0}(A)=\int_{\widehat{A B}} \frac{d \mathcal{E}_{0}}{d t} d t=\int_{\widehat{A B}}-|y| y^{2} d t<0
$$

implying that $E_{0}$ is a stable nilpotent focus when $\mu_{1}=0$ and $\mu_{2}=0$.

Based on Lemmas 2.1 and 2.2, we investigate bifurcations from finite equilibria in the following three propositions.

Proposition 2.1. Consider $\mu_{1}>0$. There is a unique limit cycle occurring in a small neighborhood of $E_{l}$ (resp. $E_{r}$ ) if $\mu_{2}$ varies from $\mu_{2}=0$ to $\mu_{2}=-\epsilon$ and no limit cycles in any small neighborhood of $E_{l}$ (resp. $E_{r}$ ) if $0 \leq \mu_{2}<\epsilon$, where $\epsilon>0$ is sufficiently small. Moreover, the limit cycles are stable.

Proof. Applying the transformation $(x, y, t) \rightarrow\left(x+\sqrt{\mu_{1}}, y+\sqrt{\mu_{1}}, t\right)$, which translates $E_{r}$ to the origin, system $(1.2 \mathrm{~b})$ is changed into

$$
\dot{x}=y, \quad \dot{y}=-2 \mu_{1} x-\mu_{2} y-|y| y-3 \sqrt{\mu_{1}} x^{2}-x^{3} .
$$

By the following transformation

$$
(x, y, t) \rightarrow\left(\frac{x}{2 \mu_{1}}-\frac{\mu_{2} y}{2 \mu_{1} \sqrt{8 \mu_{1}-\mu_{2}^{2}}}, \frac{2 y}{\sqrt{8 \mu_{1}-\mu_{2}^{2}}}, t\right)
$$

system (2.8) is reduced to its normal form

$$
\begin{aligned}
& \dot{x}=-\frac{\mu_{2}}{2} x+\frac{\sqrt{8 \mu_{1}-\mu_{2}^{2}}}{2} y+P_{2}(x, y)+P_{3}(x, y), \\
& \dot{y}=-\frac{\sqrt{8 \mu_{1}-\mu_{2}^{2}}}{2} x-\frac{\mu_{2}}{2} y+Q_{2}(x, y)+Q_{3}(x, y),
\end{aligned}
$$

where

$$
\begin{aligned}
P_{2}(x, y) & =-\frac{3 \mu_{2}}{8 \mu_{1}^{3 / 2}} x^{2}+\frac{3 \mu_{2}^{2}}{4 \mu_{1}^{3 / 2} \sqrt{8 \mu_{1}-\mu_{2}^{2}}} x y-\frac{3 \mu_{2}^{3}}{8 \mu_{1}^{3 / 2}\left(8 \mu_{1}-\mu_{2}^{2}\right)} y^{2}-\frac{2 \mu_{2}}{8 \mu_{1}-\mu_{2}^{2}}|y| y, \\
Q_{2}(x, y) & =-\frac{3 \sqrt{8 \mu_{1}-\mu_{2}^{2}}}{8 \mu_{1}^{3 / 2}} x^{2}+\frac{3 \mu_{2}}{4 \mu_{1}^{3 / 2}} x y-\frac{3 \mu_{2}^{2}}{8 \mu_{1}^{3 / 2} \sqrt{8 \mu_{1}-\mu_{2}^{2}}} y^{2}-\frac{2}{\sqrt{8 \mu_{1}-\mu_{2}^{2}}}|y| y,
\end{aligned}
$$

$P_{3}(x, y)=O\left(\left(\sqrt{x^{2}+y^{2}}\right)^{3}\right)$ and $Q_{3}(x, y)=O\left(\left(\sqrt{x^{2}+y^{2}}\right)^{3}\right)$. Taking the polar coordinates $x=$ $r \cos \theta$ and $y=r \sin \theta$, we have

$$
\frac{d r}{d \theta}=\frac{-\mu_{2} r / 2+H_{2}(\theta) r^{2}+O\left(r^{3}\right)}{-\sqrt{8 \mu_{1}-\mu_{2}^{2}} / 2+G_{1}(\theta) r+O\left(r^{2}\right)},
$$

where

$$
H_{2}(\theta)=-\frac{3 \mu_{2} \cos ^{3} \theta}{8 \mu_{1}^{3 / 2}}-\frac{3\left(8 \mu_{1}-3 \mu_{2}^{2}\right) \cos ^{2} \theta \sin \theta}{8 \mu_{1}^{3 / 2} \sqrt{8 \mu_{1}-\mu_{2}^{2}}}+\frac{3\left(16 \mu_{1}-3 \mu_{2}^{2}\right) \mu_{2} \cos \theta \sin ^{2} \theta}{8 \mu_{1}^{3 / 2}\left(8 \mu_{1}-\mu_{2}^{2}\right)}
$$




$$
\begin{aligned}
& -\frac{3 \mu_{2}^{2} \sin ^{3} \theta}{8 \mu_{1}^{3 / 2} \sqrt{8 \mu_{1}-\mu_{2}^{2}}}-\frac{2 \mu_{2} \cos \theta \sin \theta|\sin \theta|}{8 \mu_{1}-\mu_{2}^{2}}-\frac{2 \sin ^{2} \theta|\sin \theta|}{\sqrt{8 \mu_{1}-\mu_{2}^{2}}}, \\
G_{1}(\theta)= & -\frac{3 \sqrt{8 \mu_{1}-\mu_{2}^{2}} \cos ^{3} \theta}{8 \mu_{1}^{3 / 2}}+\frac{9 \mu_{2} \cos ^{2} \theta \sin \theta}{8 \mu_{1}^{3 / 2}}-\frac{9 \mu_{2}^{2} \cos \theta \sin ^{2} \theta}{8 \mu_{1}^{3 / 2} \sqrt{8 \mu_{1}-\mu_{2}^{2}}}+\frac{3 \mu_{2}^{3} \sin ^{3} \theta}{8 \mu_{1}^{3 / 2}\left(8 \mu_{1}-\mu_{2}^{2}\right)} \\
& -\frac{2 \cos \theta \sin \theta|\sin \theta|}{\sqrt{8 \mu_{1}-\mu_{2}^{2}}}+\frac{2 \mu_{2} \sin ^{2} \theta|\sin \theta|}{8 \mu_{1}-\mu_{2}^{2}} .
\end{aligned}
$$

One can introduce a new variable $\rho$, satisfying

$$
r=\rho-\frac{3 \pi \cos ^{3} \theta+8 \sqrt{\mu_{1}} \theta-3 \pi-2 \operatorname{sgn}(\theta)\left(\pi \sqrt{\mu_{1}} \cos ^{3} \theta-3 \pi \sqrt{\mu_{1}} \cos \theta-3 \theta+2 \pi \sqrt{\mu_{1}}\right)}{12 \pi \mu_{1}^{3 / 2}} \rho^{2},
$$

for $-\pi \leq \theta \leq \pi$, to transform (2.9) with $\mu_{2}=0$ to its normal form equation

$$
\frac{d \rho}{d \theta}=\frac{4 \sqrt{\mu_{1}}+3 \operatorname{sgn}(\theta)}{6 \pi \mu_{1} \sqrt{\mu_{1}}} \rho^{2}+O\left(\rho^{3}\right), \quad-\pi \leq \theta \leq \pi .
$$

By the same change of the variable, we can transform (2.9) with general $\mu_{2}$ to the form

$$
\frac{d \rho}{d \theta}=\frac{\mu_{2} \rho}{\sqrt{8 \mu_{1}-\mu_{2}^{2}}}+\left(\frac{128 \mu_{1}+96 \operatorname{sgn}(\theta) \sqrt{\mu_{1}}}{3 \pi\left(8 \mu_{1}-\mu_{2}^{2}\right)^{2}}+O(u)\right) \rho^{2}+O\left(\rho^{3}\right), \quad-\pi \leq \theta \leq \pi .
$$

Suppose that the function

$$
R\left(\rho_{0}, \theta, \mu_{2}\right)=u_{1}\left(\theta, \mu_{2}\right) \rho_{0}+u_{2}\left(\theta, \mu_{2}\right) \rho_{0}^{2}+O\left(\rho_{0}^{3}\right), \quad-\pi \leq \theta \leq \pi,
$$

is the solution of (2.11) satisfying the initial condition $R\left(\rho_{0}, 0, \mu_{2}\right)=\rho_{0}$. Then

$$
u_{1}\left(0, \mu_{2}\right)=1 \quad \text { and } \quad u_{2}\left(0, \mu_{2}\right)=0
$$

Moreover, $R\left(\rho_{0}, \theta, 0\right)$ is the solution of (2.10) satisfying the initial condition $R\left(\rho_{0}, 0,0\right)=\rho_{0}$. A calculation shows that

$$
R\left(\rho_{0}, \theta, \mu_{2}\right)=\exp \left(\frac{\mu_{2} \theta}{\sqrt{8 \mu_{1}-\mu_{2}^{2}}}\right) \rho_{0}+u_{2}\left(\theta, \mu_{2}\right) \rho_{0}^{2}+O\left(\rho_{0}^{3}\right)
$$

and

$$
R\left(\rho_{0}, \theta, 0\right)=\rho_{0}+\frac{4 \sqrt{\mu_{1}} \theta+3 \theta \operatorname{sgn}(\theta)}{6 \pi \mu_{1} \sqrt{\mu_{1}}} \rho_{0}^{2}+O\left(\rho_{0}^{3}\right) .
$$

We define the Poincaré map $\mathcal{P}\left(x, \mu_{2}\right)$ along the $x$-axis for the system $(2.11)$, and let

$$
V\left(x, \mu_{2}\right)=\mathcal{P}\left(R\left(x,-\pi, \mu_{2}\right), \mu_{2}\right)-R\left(x,-\pi, \mu_{2}\right)
$$

Then the number of periodic orbits of system (2.11) near $x=0$ for sufficiently small $\mu_{2}$ is determined by the number of zeros of $V\left(x, \mu_{2}\right)$ for $x>0$. When $x>0$, we have

$$
V\left(x, \mu_{2}\right)=R\left(x, \pi, \mu_{2}\right)-R\left(x,-\pi, \mu_{2}\right) .
$$

From (2.12) and (2.13),

$$
V\left(x, \mu_{2}\right)=x \tilde{V}\left(x, \mu_{2}\right)
$$


where

$$
\tilde{V}\left(x, \mu_{2}\right)=\exp \left(\frac{\mu_{2} \pi}{\sqrt{8 \mu_{1}-\mu_{2}^{2}}}\right)-\exp \left(-\frac{\mu_{2} \pi}{\sqrt{8 \mu_{1}-\mu_{2}^{2}}}\right)+\left(u_{2}\left(\pi, \mu_{2}\right)-u_{2}\left(-\pi, \mu_{2}\right)\right) x+O\left(x^{2}\right)
$$

and

$$
\tilde{V}(x, 0)=\frac{4}{3 \mu_{1}} x+O\left(x^{2}\right) .
$$

From (2.14), we get

$$
\frac{\partial \tilde{V}(0,0)}{\partial \mu_{2}}=\left.\frac{\partial}{\partial \mu_{2}}\left(\exp \left(\frac{\mu_{2} \pi}{\sqrt{8 \mu_{1}-\mu_{2}^{2}}}\right)-\exp \left(-\frac{\mu_{2} \pi}{\sqrt{8 \mu_{1}-\mu_{2}^{2}}}\right)\right)\right|_{\mu_{2}=0}=\frac{\pi}{\sqrt{2 \mu_{1}}} \neq 0 .
$$

By the implicit function theorem, there is a unique smooth function $\mu_{2}=\mu_{2}(x)$ for $|x|<\epsilon$, such that $\mu_{2}(0)=0$ and $\tilde{V}\left(x, \mu_{2}(x)\right) \equiv 0$. Differentiating it with respect to $x$, we have

$$
\frac{\partial \tilde{V}}{\partial x}+\frac{\partial \tilde{V}}{\partial \mu_{2}} \mu_{2}^{\prime}(x)=0
$$

It follows from (2.15) that

$$
\mu_{2}^{\prime}(0)=-\frac{4 \sqrt{2}}{3 \pi \sqrt{\mu_{1}}}<0
$$

Then $\mu_{2}(x)<0$ when $x>0$ and one can get the inverse function $x=x\left(\mu_{2}\right)$ of $\mu_{2}=\mu_{2}(x)$. Thus, there exist $\sigma>0$ and $\eta>0$ such that among all the orbits of $(2.11)$ crossing the interval $(0, \eta)$ on the $x$-axis, only the orbit passing through the point $\left(x\left(\mu_{2}\right), 0\right)$ for $-\sigma<\mu_{2}<0$ is periodic. The stability of the periodic orbit is obtained from $(2.9)$.

Similar to Proposition 2.1, a generalized Hopf bifurcation from $E_{0}$ will occur when $\mu_{1}<0$ and $\mu_{2}$ varies from $\mu_{2}=0$ to $\mu_{2}=-\epsilon$, where $\epsilon>0$ is sufficiently small. Notice that $E_{0}$ is a stable nilpotent focus when $\mu_{1}=\mu_{2}=0$, where limit cycles may also be bifurcated. The following proposition presents a generalized Hopf bifurcation from $E_{0}$, which is available in both cases $\mu_{1}<0$ and $\mu_{1}=0$.

Proposition 2.2. Consider $\mu_{1} \leq 0$. There is a unique limit cycle occurring in a small neighborhood of $E_{0}$ if $\mu_{2}$ varies from $\mu_{2}=0$ to $\mu_{2}=-\epsilon$ and no limit cycles in any small neighborhood of $E_{0}$ if $\mu_{2} \geq 0$, where $\epsilon>0$ is sufficiently small. Moreover, the limit cycle is stable.

Proof. When $\mu_{1} \leq 0$ and $\mu_{2}<0$, the existence, uniqueness and stability of limit cycles can be obtained by verifying conditions (1)-(3) in [6, Propostion 2.1]. Clearly, $g(x)$ and $f(y)$ in $[6$, Propostion 2.1] are assigned to be $-\mu_{1} x+x^{3}$ and $\mu_{2}+|y|$ respectively for system (1.2b). One can check that $g(x)$ is odd and increasing when $\mu_{1} \leq 0$. Then the condition (1) holds. Choose $y_{0}=-\mu_{2}>0$. It is easy to see that $f(y)$ is even, $f(y)<0$ if $0<y<y_{0}$ and $f(y)>0$ and increasing if $y>y_{0}$. Then the condition (2) holds. Let

$$
G(x)=\int_{0}^{x} g(s) d s=-\frac{1}{2} \mu_{1} x^{2}+\frac{1}{4} x^{4} .
$$

Obviously, $\lim _{x \rightarrow \infty} G(x)=\infty$. Then the condition (3) holds. 
When $\mu_{1} \leq 0$ and $\mu_{2} \geq 0$,

$$
\operatorname{div}\left(y, \mu_{1} x-x^{3}-\left(\mu_{2}+|y|\right) y=-\mu_{2}-2|y| \leq 0 .\right.
$$

By Bendixson-Dulac Criterion, system (1.2b) exhibits no limit cycles.

Therefore, when $\mu_{1} \leq 0$ system (1.2b) exhibits a unique limit cycle if $-\epsilon<\mu_{2}<0$ and no limit cycles if $\mu_{2} \geq 0$, where $\epsilon>0$ is sufficiently small. Since $E_{0}$ is the unique finite equilibrium of system $(1.2 \mathrm{~b})$, the limit cycle must surround $E_{0}$ if it exists. Notice that the vector field of system $(1.2 \mathrm{~b})$ is rotated with respect to $\mu_{2}$ by $[14,21]$ and system $(1.2 \mathrm{~b})$ exhibits no limit cycles for $\mu_{2}=0$. Assume that the unique limit cycle of system (1.2b) does not lie in a small neighborhood of $E_{0}$ when $\mu_{2}=-\epsilon$, where $\epsilon>0$ is sufficiently small. By the rotated property, the unique stable limit cycle still persists when $\mu_{2}=0$. This is a contradiction, which completes the proof.

Proposition 2.3. The bifurcation diagram of system (1.2b) includes the following bifurcation curves:

(a) pitchfork bifurcation curve $P:=\left\{\left(\mu_{1}, \mu_{2}\right) \in \mathbb{R}^{2}: \mu_{1}=0\right\}$;

(b) generalized Hopf bifurcation curves $H_{1}:=\left\{\left(\mu_{1}, \mu_{2}\right) \in \mathbb{R}^{2}: \mu_{1}>0, \mu_{2}=0\right\}$ for $E_{l}$ and $E_{r}$, and $H_{2}:=\left\{\left(\mu_{1}, \mu_{2}\right) \in \mathbb{R}^{2}: \mu_{1} \leq 0, \mu_{2}=0\right\}$ for $E_{0}$.

Proof. By Lemmas 2.1 and 2.2, the number of equilibria at finity varies from 3 to 1 when $\mu_{1}$ varies from a positive value to a non-positive one. Then $\mu_{1}=0$ is the pitchfork bifurcation curve. Thus, (a) is proven.

When $\mu_{1}>0$, by Proposition 2.1 the stable weak foci $E_{l}$ and $E_{r}$ become unstable rough foci and two limit cycles occur at the same time, one is in a small neighborhood of $E_{l}$ and the other is in a small neighborhood of $E_{r}$, as $\mu_{2}$ changes from 0 to a negative value. Then $H_{1}$ is a generalized Hopf bifurcation curve. When $\mu_{1}<0$ (resp. $\mu_{1}=0$ ), by Proposition 2.2, $E_{0}$ becomes an unstable rough focus (resp. unstable degenerate node) from the stable weak focus (resp. stable nilpotent focus) and one stable limit cycle occurs in a small neighborhood of $E_{0}$ as $\mu_{2}$ changes to a negative value from 0 . Then $H_{2}$ is a generalized Hopf bifurcation curve and (b) is proven.

To see the behavior of orbits when either $|x|$ or $|y|$ is large, we need to discuss the possible equilibria at infinity. By Poincaré transformations $x=1 / z, y=u / z$ and $x=v / z, y=1 / z$, system (1.2b) can be rewritten as

$$
\frac{d u}{d \tau}=-1+\mu_{1} z^{2}-\mu_{2} u z^{2}-u|u z|-u^{2} z^{2}, \quad \frac{d z}{d \tau}=-u z^{3}
$$

and

$$
\frac{d v}{d \tau}=v|z|+z^{2}+\mu_{2} v z^{2}+v^{4}-\mu_{1} v^{2} z^{2}, \quad \frac{d z}{d \tau}=z|z|+\mu_{2} z^{3}+v^{3} z-\mu_{1} v z^{3},
$$

where $d \tau=z^{2} d t$. Obviously, system (2.16) has no equilibria on the $u$-axis and $D:(0,0)$ is a equilibrium of system (2.17). Moreover, $D$ corresponds to a pair of equilibria $I_{y}^{+}$and $I_{y}^{-}$at infinity of system $(1.2 \mathrm{~b})$, which lie on the positive $y$-axis and negative $y$-axis respectively. The following lemma exhibits the qualitative properties of $I_{y}^{+}$and $I_{y}^{-}$. 
Lemma 2.3. $I_{y}^{+}$and $I_{y}^{-}$are degenerate saddle-nodes. Moreover, for system (1.2b) the direction of the orbit on the boundary of the Poincaré disc is clockwise and there are infinitely many orbits leaving $I_{y}^{+}$in the direction of the negative $x$-axis and infinitely many orbits leaving $I_{y}^{-}$in the direction of the positive $x$-axis as $t \rightarrow \infty$.

Proof. Since system (2.17) is invariant under the transformation $(v, z) \rightarrow(v,-z)$, it is symmetric about the $v$-axis. So it is enough to consider qualitative properties of $D$ in the upper half $v z$-plane, i.e., $z \geq 0$. Taking polar coordinates $x=r \cos \theta$ and $y=r \sin \theta$, system (2.17) becomes (2.7), where $G(\theta)=-\sin ^{3} \theta$ and $H(\theta)=\cos \theta \sin ^{2} \theta+|\sin \theta|$. The equation $G(\theta)=0$ has exactly two real roots 0 and $\pi$ in the interval $[0, \pi]$. Moreover, $G(0)=H(0)=G(\pi)=H(\pi)=0$.

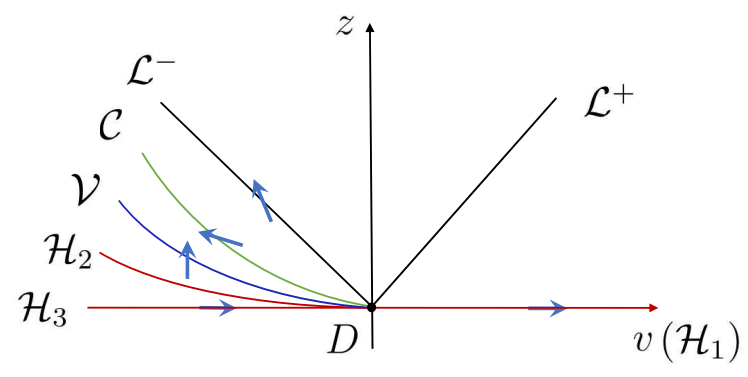

Fig. 4: Dynamical behaviors near equilibrium $D$.

From equations in (2.17), there is a unique horizontal isocline $\mathcal{H}_{1}$ which is in the upper half $v z$-plane and tangent to $\theta=0$, and there are no vertical isoclines in the open region $\Delta \widehat{\mathcal{L}}^{+D \mathcal{H}_{1}}$, where

$$
\begin{aligned}
\mathcal{H}_{1}: & =\left\{(v, z) \in \mathbb{R}^{2}: z=0,0<v<\ell\right\}, \\
\mathcal{L}^{+}: & =\left\{(v, z) \in \mathbb{R}^{2}: z=v \tan \alpha, \quad 0<v<\ell\right\}
\end{aligned}
$$

and $0<\alpha<\pi / 2$ is chosen to be arbitrarily close to 0 and $\ell>0$ is sufficiently small, as shown in Fig. 4. One can check that $\dot{v}>0$ and $\dot{z}>0$ in $\Delta \widehat{\mathcal{L}}^{+D \mathcal{H}_{1}}$ and

$$
\frac{d z}{d v}=\frac{z\left(z+\mu_{2} z^{2}+v^{3}-\mu_{1} v z^{2}\right)}{z^{2}+v\left(z+\mu_{2} z^{2}+v^{3}-\mu_{1} v z^{2}\right)}=\frac{z}{v+z^{2} /\left(z+\mu_{2} z^{2}+v^{3}-\mu_{1} v z^{2}\right)}<\frac{z}{v} .
$$

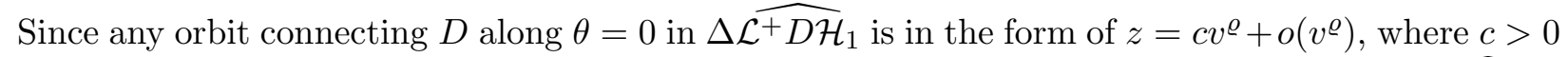
and $\varrho>1$, it is easy to compute $d z / d v=c \varrho v^{\varrho-1}+o\left(v^{\varrho-1}\right)>z / v=c v^{\varrho-1}+o\left(v^{\varrho-1}\right)$ in $\Delta{\widehat{\mathcal{L}}+D \mathcal{H}_{1}}_{1}$. Then there are no orbits connecting $D$ in the open region $\Delta \widehat{\mathcal{L}^{+} D \mathcal{H}_{1}}$. Notice that $z=0$ is an orbit of system (2.17) and $\left.\dot{v}\right|_{z=0}=v^{4}>0$. There is only one orbit connecting $D$ in the direction $\theta=0$, which is exactly $z=0$ and $v>0$. Moreover, it leaves $D$ as $t \rightarrow \infty$.

From equations in (2.17), there are two horizontal isoclines $\mathcal{H}_{2}$ and $\mathcal{H}_{3}$ and one vertical isocline $\mathcal{V}$ which are in the upper half $v z$-plane and tangent to $\theta=\pi$, where

$$
\mathcal{H}_{2}:=\left\{(v, z) \in \mathbb{R}^{2}: z=-v^{3}+O\left(v^{6}\right), \quad-\ell<v<0\right\},
$$




$$
\begin{aligned}
\mathcal{H}_{3}: & =\left\{(v, z) \in \mathbb{R}^{2}: z=0, \quad-\ell<v<0\right\}, \\
\mathcal{V}: & =\left\{(v, z) \in \mathbb{R}^{2}: z=-v^{3}-v^{5}+O\left(v^{6}\right), \quad-\ell<v<0\right\},
\end{aligned}
$$

where $\ell>0$ is sufficiently small. Obviously, $\mathcal{V}$ is above $\mathcal{H}_{2}$, as shown in Fig. 4 . One can get that

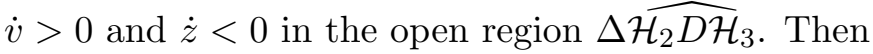

$$
\frac{d z}{d v}=\frac{z\left(z+\mu_{2} z^{2}+v^{3}-\mu_{1} v z^{2}\right)}{z^{2}+v\left(z+\mu_{2} z^{2}+v^{3}-\mu_{1} v z^{2}\right)}=\frac{z}{v+z^{2} /\left(z+\mu_{2} z^{2}+v^{3}-\mu_{1} v z^{2}\right)}>\frac{z}{v} .
$$

Since any orbit connecting $D$ along $\theta=\pi$ in $\Delta{\widehat{\mathcal{H}} 2 \widehat{\mathcal{H}}_{3}}_{3}$ is in the form of $z=c v^{\varrho}+o\left(v^{\varrho}\right)$, where $\varrho>1$ and $c>0(<0)$ if $\varrho$ is even (odd), one can compute $d z / d v=c \varrho v^{\varrho-1}+o\left(v^{\varrho-1}\right)<z / v=$

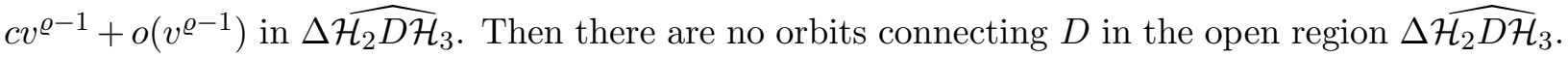
It is easy to check that $\dot{v}>0$ and $\dot{z}>0$ in the open region $\Delta \widehat{\mathcal{V D \mathcal { H } _ { 2 }}}$. There are also no orbits connecting $D$ in $\Delta \widehat{\mathcal{V D H}}_{2}$ because any orbit connecting $D$ in second quadrant has a negative slope. Let

$$
\mathcal{L}^{-}:=\left\{(v, z) \in \mathbb{R}^{2}: z=-v \tan \alpha, \quad-\ell<v<0\right\},
$$

where $0<\alpha<\pi / 2$ is chosen to be arbitrarily close to 0 and $\ell>0$ is sufficiently small. Notice that $\dot{v}<0, \dot{z}>0$,

$$
\left.\dot{z}\right|_{\mathcal{V}}=v^{8}+O\left(v^{10}\right)>0 \text { and }\left.\quad \frac{d z}{d v}\right|_{\mathcal{L}^{-}}=\frac{\tan ^{2} \alpha}{\tan ^{2} \alpha-\tan \alpha}+O(v)<-\tan \alpha
$$

in the open region $\Delta \widehat{\mathcal{L}^{-D V}}$. Then $\Delta \widehat{\mathcal{L}^{-} D \mathcal{V}}$ is a generalized normal sector of Class III, as shown in Fig. 4. Moreover, let

$$
\mathcal{C}:=\left\{(v, z) \in \mathbb{R}^{2}: z=v^{2}, \quad-\ell<v<0\right\}
$$

Since

$$
\left.\frac{d z}{d v}\right|_{\mathcal{C}}=v+O\left(v^{2}\right)>2 v
$$

and $\mathcal{C}$ is in the region $\Delta \widehat{\mathcal{L}-D \mathcal{V}}$. Then $\Delta \widehat{\mathcal{L}-D \mathcal{V}}$ covers a generalized normal sector of Class I, which is $\Delta \widehat{\mathcal{L}-D \mathcal{C}}$, as shown in Fig. 4. Thus, there are infinitely many orbits leaving $D$ along $\theta=\pi$ in the region $\Delta \widehat{\mathcal{L}^{-} D \mathcal{V}}$ as $\tau \rightarrow \infty$. Combining the facts that $z=0$ is also an orbit of system (2.17) and $\left.\dot{v}\right|_{z=0}=v^{4}$, there is only one orbit approaching $D$, which is exactly $z=0$ and $v<0$. Therefore, the properties of $I_{y}^{+}$and $I_{y}^{-}$for system $(1.2 \mathrm{~b})$ are shown in Fig. 5.

\section{$3 \quad$ Limit cycles and homoclinic loops}

In this section, we investigate the existence and the number of limit cycles and homoclinic loops for system (1.2b). For simplicity, the whole parameter space is divided into the following four subsets:
$(\mathbf{c 1}):\left\{\begin{array}{l}\mu_{1} \in \mathbb{R} \\ \mu_{2} \geq 0\end{array}\right.$
$(\mathbf{c 2}):\left\{\begin{array}{l}\mu_{1} \leq 0, \\ \mu_{2}<0,\end{array}\right.$
$(\mathbf{c 3}):\left\{\begin{array}{l}\mu_{1}>0, \\ \mu_{2} \leq \frac{-2\left(12 \mu_{1}^{3}\right)^{1 / 4}}{3},\end{array}\right.$
(c4) : $\left\{\begin{array}{l}\mu_{1}>0, \\ \frac{-2\left(12 \mu_{1}^{3}\right)^{1 / 4}}{3}<\mu_{2}<0 .\end{array}\right.$

Lemma 3.1. When (c1) holds, system (1.2b) exhibits neither limit cycles nor homoclinic loops. 


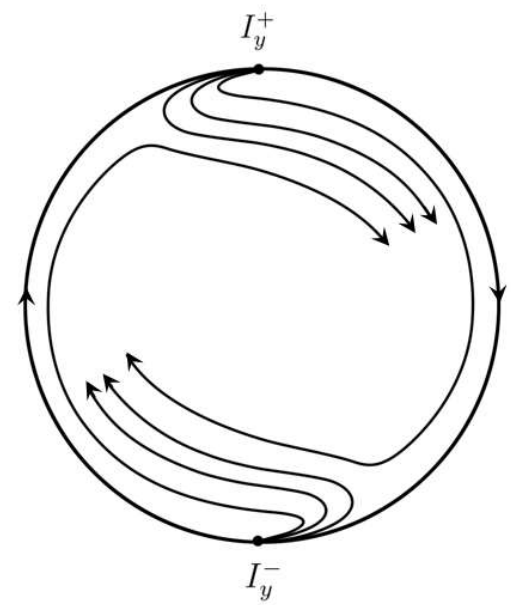

Fig. 5: Dynamical behaviors near $I_{y}^{+}$and $I_{y}^{+}$.

Proof. When (c1) holds, one can calculate the divergence of system (1.2b),

$$
\operatorname{div}\left(y, \mu_{1} x-x^{3}-\left(\mu_{2}+|y|\right) y\right)=-\mu_{2}-2|y| \leq 0 .
$$

By Bendixson-Dulac Criterion, system (1.2b) has no closed orbits, implying the nonexistence of limit cycles and homoclinic loops.

Lemma 3.2. When (c2) holds, there is a unique limit cycle for system (1.2b). Moreover, the limit cycle is stable.

Proof. When (c2) holds, one can check that conditions (1)- (3) in [6, Propostion 2.1] are satisfied with $g(x)=-\mu_{1} x+x^{3}$ and $f(y)=\mu_{2}+|y|$, as in the proof of Proposition 2.2. Then system (1.2b) exhibits a unique limit cycle, which is stable.

For the remaining two cases, which is a division of the forth quadrant of the $\left(\mu_{1}, \mu_{2}\right)$-plane, by Lemma 2.1 system $(1.2 \mathrm{~b})$ has three equilibria and $E_{0}$ is a saddle. Then the limit cycle bifurcation or the homoclinic bifurcation may occur. Since system $(1.2 \mathrm{~b})$ is symmetric with respect to $E_{0}$ and $E_{0}$ is a saddle, limit cycles can surround all three equilibria $E_{0}, E_{l}$ and $E_{r}$ or surround one of equilibria $E_{l}$ and $E_{r}$. In what follows, let large limit cycles be the ones surrounding all three equilibria $E_{0}, E_{l}$ and $E_{r}$, and small limit cycles be the ones surrounding $E_{l}$ or $E_{r}$ for simplicity.

To study the existence and number of closed orbits, we consider horizontal and vertical isoclines of system (1.2b). Clearly, the vertical isocline of system $(1.2 \mathrm{~b})$ is the $x$-axis. The horizontal isocline $\mu_{1} x+x^{3}-\left(\mu_{2}+|y|\right) y=0$ depends on the parameters $\mu_{1}$ and $\mu_{2}$.

Lemma 3.3. Consider $\mu_{1}>0$ and $\mu_{2}<0$. The graphs of the horizontal isocline of system (1.2b) have the following three types:

(a) When $-2\left(12 \mu_{1}^{3}\right)^{1 / 4} / 3<\mu_{2}<0$, it is shown in Fig. 6 (a),

(b) When $\mu_{2}=-2\left(12 \mu_{1}^{3}\right)^{1 / 4} / 3$, it is shown in Fig. 6 (b), 
(c) When $\mu_{2}<-2\left(12 \mu_{1}^{3}\right)^{1 / 4} / 3$, it is shown in Fig. 6 (c).

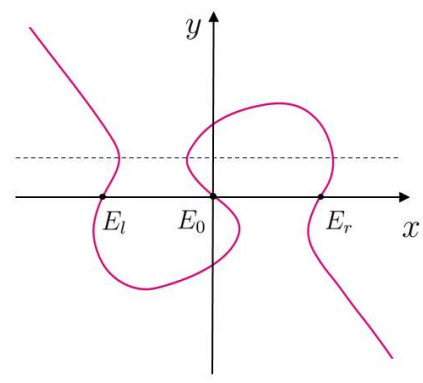

(a) for $-2\left(12 \mu_{1}^{3}\right)^{1 / 4} / 3<\mu_{2}<0$

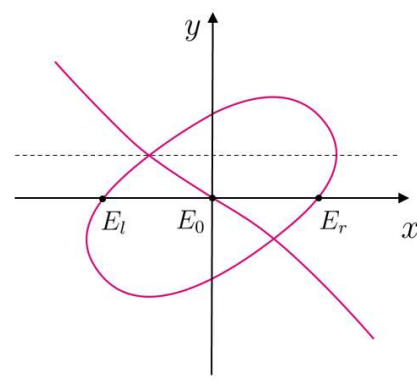

(b) for $\mu_{2}=-2\left(12 \mu_{1}^{3}\right)^{1 / 4} / 3$

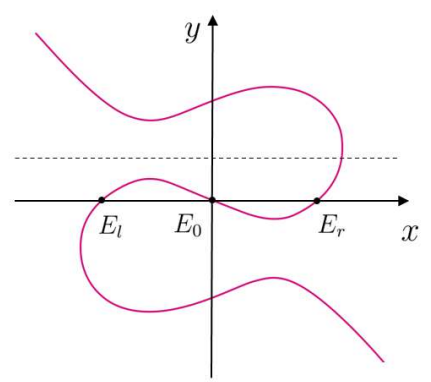

(c) for $\mu_{2}<-2\left(12 \mu_{1}^{3}\right)^{1 / 4} / 3$

Fig. 6: Graphs of the horizontal isocline of system (1.2b).

Proof. Notice that the graph of $\mu_{1} x-x^{3}-\left(\mu_{2}+|y|\right) y=0$ is symmetric about the origin. It is enough to investigate the graphs of

$$
q(x, y):=\mu_{1} x-x^{3}-\left(\mu_{2}+y\right) y=\mu_{1} x-x^{3}+\frac{\mu_{2}^{2}}{4}-\left(y+\frac{\mu_{2}}{2}\right)^{2}=0 .
$$

It is easy to see that $p(x):=\mu_{1} x-x^{3}+\mu_{2}^{2} / 4$ has a local maximum value $p_{\text {lmax }}=2\left(\mu_{1} / 3\right)^{3 / 2}+\mu_{2}^{2} / 4$ at $x=\sqrt{\mu_{1} / 3}$, a local minimum value $p_{\text {lmin }}=-2\left(\mu_{1} / 3\right)^{3 / 2}+\mu_{2}^{2} / 4$ at $x=-\sqrt{\mu_{1} / 3}$ and no other local extreme values. It follows from $\lim _{x \rightarrow \infty} p(x)=-\infty$ and $p_{\text {lmax }}>0$ that $p(x)$ has a unique zero on the interval $\left(\sqrt{\mu_{1} / 3}, \infty\right)$, denoted by $x_{1}$. Moreover, $p(x)<0$ for $x>x_{1}$.

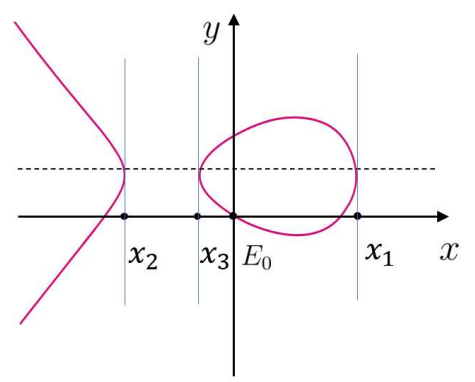

(a) for $-2\left(12 \mu_{1}^{3}\right)^{1 / 4} / 3<\mu_{2}<0$

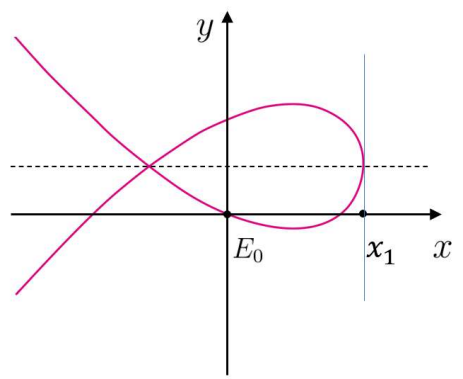

(b) for $\mu_{2}=-2\left(12 \mu_{1}^{3}\right)^{1 / 4} / 3$

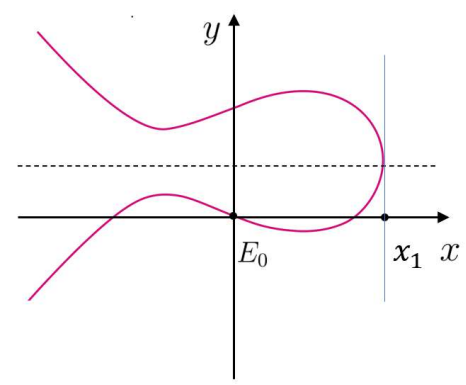

(c) for $\mu_{2}<-2\left(12 \mu_{1}^{3}\right)^{1 / 4} / 3$

Fig. 7: Graphs of $q(x, y)=0$.

When $-2\left(12 \mu_{1}^{3}\right)^{1 / 4} / 3<\mu_{2}<0$, we get $p_{\text {lmin }}<0$ and $p(x)$ has two zeros on the interval $\left(-\infty, \sqrt{\mu_{1} / 3}\right)$, denoted by $x_{2}$ and $x_{3}$. Without losing generality, assume $x_{2}<x_{3}$. Then $q(x, y) \geq 0$ implies $x \leq x_{2}$ or $x_{3} \leq x \leq x_{1}$ and the graph of $q(x, y)=0$ is shown in Fig. 7 (a).

When $\mu_{2}=-2\left(12 \mu_{1}^{3}\right)^{1 / 4} / 3$, we get $p_{\text {lmin }}=0$ and $p(x)$ has exactly one zero point on the interval $\left(-\infty, \sqrt{\mu_{1} / 3}\right)$, which is $x=-\sqrt{\mu_{1} / 3}$. Then $q(x, y) \geq 0$ implies $x \leq x_{1}$ and the graph of $q(x, y)=0$ is shown in Fig. 7 (b). 
When $\mu_{2}<-2\left(12 \mu_{1}^{3}\right)^{1 / 4} / 3$, we get $p_{\text {lmin }}>0$ and $p(x)$ has no zeros on the interval $\left(-\infty, \sqrt{\mu_{1} / 3}\right)$. Then $q(x, y) \geq 0$ implies $x \leq x_{1}$ and the graph of $q(x, y)=0$ is shown in Fig. 7 (c).

By symmetry about the origin, we can get the graphs of the horizontal isocline of system (1.2b) are shown in Fig. 6.

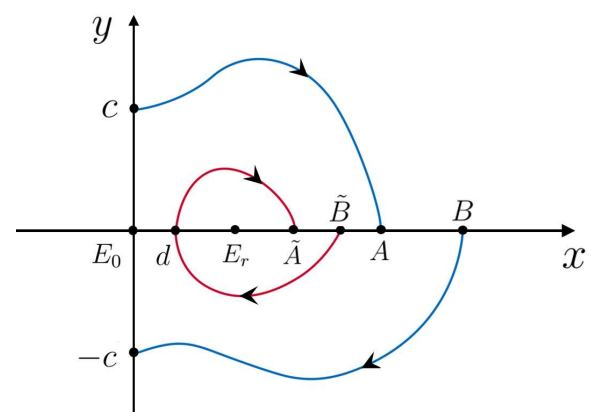

Fig. 8: Intersection points of orbits $\gamma_{\mu_{1}, \mu_{2}}^{+}(x, y)$ and $\gamma_{\mu_{1}, \mu_{2}}^{-}(x, y)$ with the $x$-axis.

Let $\gamma_{\mu_{1}, \mu_{2}}^{+}(x, y)$ (resp. $\left.\gamma_{\mu_{1}, \mu_{2}}^{-}(x, y)\right)$ be the positive (resp. negative) orbit of system $(1.2 \mathrm{~b})$ that starts at point $(x, y)$, where $x \geq 0$ and $(x, y) \neq\left(\sqrt{\mu_{1}}, 0\right)$. Specially, the orbits $\gamma_{\mu_{1}, \mu_{2}}^{+}(0,0)$ and $\gamma_{\mu_{1}, \mu_{2}}^{-}(0,0)$ are the right-hand side unstable manifold $W_{\mu_{1}, \mu_{2}}^{+}$and the right-hand side stable manifold $W_{\mu_{1}, \mu_{2}}^{-}$of system $(1.2 \mathrm{~b})$ at $E_{0}$ respectively.

Obviously, $\gamma_{\mu_{1}, \mu_{2}}^{+}(0, c)$ and $\gamma_{\mu_{1}, \mu_{2}}^{+}(d, 0)$ lie in the first quadrant at the beginning, where $c \geq 0$ and $0 \leq d<\sqrt{\mu_{1}}$. By Lemma 3.3, $\gamma_{\mu_{1}, \mu_{2}}^{+}(0, c)$ and $\gamma_{\mu_{1}, \mu_{2}}^{+}(d, 0)$ will cross the positive $x$-axis. Denote the first intersection point of $\gamma_{\mu_{1}, \mu_{2}}^{+}(0, c)$ (resp. $\left.\gamma_{\mu_{1}, \mu_{2}}^{+}(d, 0)\right)$ and the positive $x$-axis by $A:\left(x_{A}^{c}\left(\mu_{1}, \mu_{2}\right), 0\right)$ (resp. $\left.\tilde{A}:\left(x_{\tilde{A}}^{d}\left(\mu_{1}, \mu_{2}\right), 0\right)\right)$. Clearly, $x_{A}^{c}\left(\mu_{1}, \mu_{2}\right)>\sqrt{\mu_{1}}$ and $x_{\tilde{A}}^{d}\left(\mu_{1}, \mu_{2}\right)>\sqrt{\mu_{1}}$, as shown in Fig. 8 . Moreover, $x_{A}^{0}\left(\mu_{1}, \mu_{2}\right)$ and $x_{\tilde{A}}^{0}\left(\mu_{1}, \mu_{2}\right)$ are the same point.

Similarly, $\gamma_{\mu_{1}, \mu_{2}}^{-}(0,-c)$ and $\gamma_{\mu_{1}, \mu_{2}}^{-}(d, 0)$ lie in the fourth quadrant at the beginning, where $c \geq 0$ and $0 \leq d<\sqrt{\mu_{1}}$. Combining the dynamical structure of equilibria at infinity of system (1.2b), $\gamma_{\mu_{1}, \mu_{2}}^{-}(0,-c)$ and $\gamma_{\mu_{1}, \mu_{2}}^{-}(d, 0)$ will cross the positive $x$-axis or leave from $I_{y}^{-}$as $t \rightarrow \infty$. When $\gamma_{\mu_{1}, \mu_{2}}^{-}(0,-c)$ or $\gamma_{\mu_{1}, \mu_{2}}^{-}(d, 0)$ is one of the orbits leaving from $I_{y}^{-}$, we can think that it crosses the $x$-axis at positive infinity, because the infinity on the positive $x$-axis is connected with $I_{y}^{-}$by a unique orbit. Then denote the first intersection point of $\gamma_{\mu_{1}, \mu_{2}}^{-}(0,-c)\left(\operatorname{resp} . \gamma_{\mu_{1}, \mu_{2}}^{-}(d, 0)\right)$ and the positive $x$-axis by $B:\left(x_{B}^{c}\left(\mu_{1}, \mu_{2}\right), 0\right)$ (resp. $\left.\tilde{B}:\left(x_{\tilde{B}}^{d}\left(\mu_{1}, \mu_{2}\right), 0\right)\right)$. Clearly, $x_{B}^{c}\left(\mu_{1}, \mu_{2}\right)>\sqrt{\mu_{1}}$ and $x_{\tilde{B}}^{d}\left(\mu_{1}, \mu_{2}\right)>\sqrt{\mu_{1}}$, as shown in Fig. 8. Moreover, $x_{B}^{0}\left(\mu_{1}, \mu_{2}\right)$ and $x_{\tilde{B}}^{0}\left(\mu_{1}, \mu_{2}\right)$ are the same point. The following lemma presents how $x_{A}^{c}\left(\mu_{1}, \mu_{2}\right), x_{B}^{c}\left(\mu_{1}, \mu_{2}\right), x_{\tilde{A}}^{d}\left(\mu_{1}, \mu_{2}\right)$ and $x_{\tilde{B}}^{d}\left(\mu_{1}, \mu_{2}\right)$ depend continuously on $\mu_{2}$.

Lemma 3.4. Consider $\mu_{1}>0$ and $\mu_{2}<0$. For a fixed $\mu_{1}$,

(i) the ordinate $x_{A}^{c}\left(\mu_{1}, \mu_{2}\right)$ decreases continuously and the ordinate $x_{B}^{c}\left(\mu_{1}, \mu_{2}\right)$ increases continuously as $\mu_{2}$ increases, as shown in Fig. 9(a);

(ii) the ordinate $x_{\tilde{A}}^{d}\left(\mu_{1}, \mu_{2}\right)$ decreases continuously and the ordinate $x_{\tilde{B}}^{d}\left(\mu_{1}, \mu_{2}\right)$ increases continuously as $\mu_{2}$ increases, as shown in Fig. 9(b), 
where $c \geq 0$ and $0 \leq d<\sqrt{\mu_{1}}$.

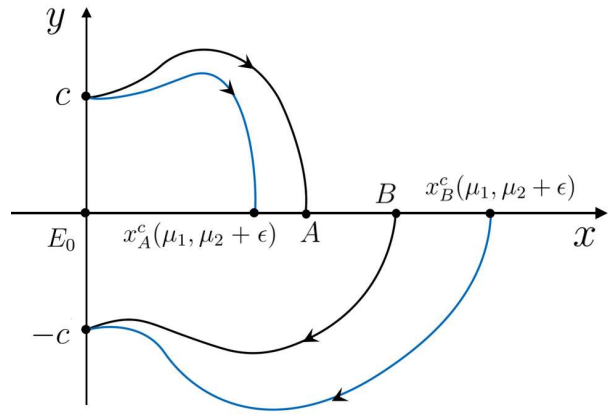

(a) Orbits passing through $(0, c)$ and $(0,-c)$

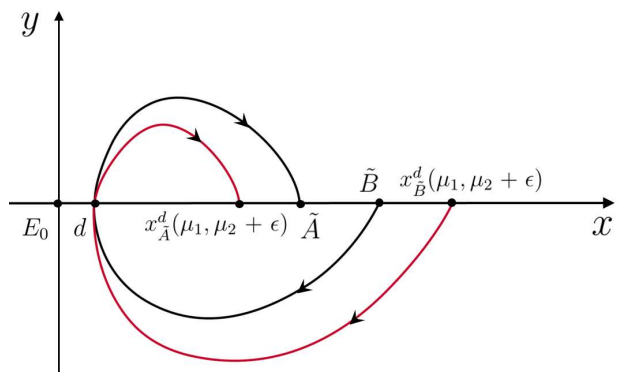

(b) Orbits passing through $(d, 0)$

Fig. 9: The orbits of system (1.2b) for $\mu_{2}$ and $\mu_{2}+\epsilon$, where $\epsilon>0$.

Proof. Let $\left(x, y_{\mu_{1}, \mu_{2}}^{+}(x)\right)$ and $\left(x, y_{\mu_{1}, \mu_{2}}^{-}(x)\right)$ denote the points on $\gamma_{\mu_{1}, \mu_{2}}^{+}(0, c)$ and $\gamma_{\mu_{1}, \mu_{2}}^{-}(0,-c)$ respectively. Then

$$
y_{\mu_{1}, \mu_{2}}^{+}(0)=c, \quad y_{\mu_{1}, \mu_{2}}^{-}(0)=-c, \quad y_{\mu_{1}, \mu_{2}}^{+}\left(x_{A}^{c}\left(\mu_{1}, \mu_{2}\right)\right)=0 \quad \text { and } \quad y_{\mu_{1}, \mu_{2}}^{-}\left(x_{B}^{c}\left(\mu_{1}, \mu_{2}\right)\right)=0 .
$$

Let $z_{\mu_{1}, \mu_{2}}^{+}(x)=y_{\mu_{1}, \mu_{2}+\epsilon}^{+}(x)-y_{\mu_{1}, \mu_{2}}^{+}(x)$ for $0 \leq x \leq \min \left\{x_{A}^{c}\left(\mu_{1}, \mu_{2}\right), x_{A}^{c}\left(\mu_{1}, \mu_{2}+\epsilon\right)\right\}$ be the vertical distance between $\gamma_{\mu_{1}, \mu_{2}+\epsilon}^{+}(0, c)$ and $\gamma_{\mu_{1}, \mu_{2}}^{+}(0, c)$, where $\left(x_{A}^{c}\left(\mu_{1}, \mu_{2}+\epsilon\right), 0\right)$ is the first intersection point of $\gamma_{\mu_{1}, \mu_{2}+\epsilon}^{+}(0, c)$ and the positive $x$-axis and $|\epsilon|$ is sufficiently small. One can check that

$$
\begin{aligned}
z_{\mu_{1}, \mu_{2}}^{+}(x) & =\left.\left\{y_{\mu_{1}, \mu_{2}+\epsilon}^{+}(s)-y_{\mu_{1}, \mu_{2}}^{+}(s)\right\}\right|_{0} ^{x} \\
& =\int_{0}^{x}\left\{\left(\frac{\mu_{1} s-s^{3}}{y_{\mu_{1}, \mu_{2}+\epsilon}^{+}(s)}-\left(\mu_{2}+\epsilon\right)-\left|y_{\mu_{1}, \mu_{2}+\epsilon}^{+}(s)\right|\right)-\left(\frac{\mu_{1} s-s^{3}}{y_{\mu_{1}, \mu_{2}}^{+}(s)}-\mu_{2}-\left|y_{\mu_{1}, \mu_{2}}^{+}(s)\right|\right)\right\} d s \\
& =H_{1}(x)+H_{2}(x),
\end{aligned}
$$

where

$$
H_{1}(x)=-\epsilon x, \quad H_{2}(x)=\int_{0}^{x} z_{\mu_{1}, \mu_{2}}^{+}(s) H_{3}(s) d s \quad \text { and } \quad H_{3}(x)=\frac{-\mu_{1} x+x^{3}}{y_{\mu_{1}, \mu_{2}+\epsilon}^{+}(x) y_{\mu_{1}, \mu_{2}}^{+}(x)}-1 .
$$

From (3.1), we get

$$
z_{\mu_{1}, \mu_{2}}^{+}(x) H_{3}(x)=H_{1}(x) H_{3}(x)+H_{2}(x) H_{3}(x) .
$$

It follows from (3.2) that

$$
\frac{d H_{2}(x)}{d x}-H_{2}(x) H_{3}(x)=H_{1}(x) H_{3}(x),
$$

which is a first order linear differential equation. With the initial condition $H_{2}(0)=0$, we get

$$
H_{2}(x)=\int_{0}^{x} H_{1}(\tau) H_{3}(\tau) \exp \left\{\int_{\tau}^{x} H_{3}(\eta) d \eta\right\} d \tau
$$

From (3.1) and (3.3),

$$
z_{\mu_{1}, \mu_{2}}^{+}(x)=H_{1}(x)+\int_{0}^{x} H_{1}(\tau) H_{3}(\tau) \exp \left\{\int_{\tau}^{x} H_{3}(\eta) d \eta\right\} d \tau
$$




$$
\begin{aligned}
& =H_{1}(x)-\left[H_{1}(\tau) \exp \left\{\int_{\tau}^{x} H_{3}(\eta) d \eta\right\}\right]_{0}^{x}+\int_{0}^{x} H_{1}^{\prime}(\tau) \exp \left\{\int_{\tau}^{x} H_{3}(\eta) d \eta\right\} d \tau \\
& =H_{1}(0) \exp \left\{\int_{0}^{x} H_{3}(\eta) d \eta\right\}+\int_{0}^{x} H_{1}^{\prime}(\tau) \exp \left\{\int_{\tau}^{x} H_{3}(\eta) d \eta\right\} d \tau \\
& =-\epsilon \int_{0}^{x} \exp \left\{\int_{\tau}^{x} H_{3}(\eta) d \eta\right\} d \tau<0(\text { resp. }>0)
\end{aligned}
$$

if $\epsilon>0$ (resp. <0). Then $0=y_{\mu_{1}, \mu_{2}+\epsilon}^{+}\left(x_{A}^{c}\left(\mu_{1}, \mu_{2}+\epsilon\right)\right)<y_{\mu_{1}, \mu_{2}}^{+}\left(x_{A}^{c}\left(\mu_{1}, \mu_{2}+\epsilon\right)\right)$ when $\epsilon>0$, implying that $x_{A}^{c}\left(\mu_{1}, \mu_{2}+\epsilon\right)<x_{A}^{c}\left(\mu_{1}, \mu_{2}\right)$. Moreover, it follows from (3.4) that $\lim _{\epsilon \rightarrow 0} z_{\mu_{1}, \mu_{2}}^{+}(x)=0$. That means $\lim _{\epsilon \rightarrow 0} x_{A}^{c}\left(\mu_{1}, \mu_{2}+\epsilon\right)=x_{A}^{c}\left(\mu_{1}, \mu_{2}\right)$. Thus $x_{A}^{c}\left(\mu_{1}, \mu_{2}\right)$ decreases continuously as $\mu_{2}$ increases.

Let $z_{\mu_{1}, \mu_{2}}^{-}(x)=y_{\mu_{1}, \mu_{2}+\epsilon}^{-}(x)-y_{\mu_{1}, \mu_{2}}^{-}(x)$ for $0 \leq x \leq \min \left\{x_{B}^{c}\left(\mu_{1}, \mu_{2}\right), x_{B}^{c}\left(\mu_{1}, \mu_{2}+\epsilon\right)\right\}$, where $\left(x_{B}^{c}\left(\mu_{1}, \mu_{2}+\epsilon\right), 0\right)$ is the first intersection point of $\gamma_{\mu_{1}, \mu_{2}+\epsilon}^{-}(0,-c)$ and the positive $x$-axis and $|\epsilon|$ is sufficiently small. Similar to the calculation of $z_{\mu_{1}, \mu_{2}}^{+}(x)$, we get

$$
z_{\mu_{1}, \mu_{2}}^{-}(x)=-\epsilon \int_{0}^{x} \exp \left\{\int_{\tau}^{x} \hat{H}_{3}(\eta) d \eta\right\} d \tau<0(\text { resp. }>0) \text {, if } \epsilon>0(\text { resp. }<0),
$$

where $\hat{H}_{3}(x)=\left(-\mu_{1} x+x^{3}\right) /\left(y_{\mu_{1}, \mu_{2}+\epsilon}^{-}(x) y_{\mu_{1}, \mu_{2}}^{-}(x)\right)+1$. Then when $\epsilon>0$ we have $y_{\mu_{1}, \mu_{2}+\epsilon}^{-}\left(x_{B}^{c}\left(\mu_{1}, \mu_{2}\right)\right)$ $<y_{\mu_{1}, \mu_{2}}^{-}\left(x_{B}^{c}\left(\mu_{1}, \mu_{2}\right)\right)=0$, implying that $x_{B}^{c}\left(\mu_{1}, \mu_{2}+\epsilon\right)>x_{B}^{c}\left(\mu_{1}, \mu_{2}\right)$. Moreover, it follows from (3.5) that $\lim _{\epsilon \rightarrow 0} z_{\mu_{1}, \mu_{2}}^{-}(x)=0$. That implies that $\lim _{\epsilon \rightarrow 0} x_{B}^{c}\left(\mu_{1}, \mu_{2}+\epsilon\right)=x_{B}^{c}\left(\mu_{1}, \mu_{2}\right)$. Hence, $x_{B}^{c}\left(\mu_{1}, \mu_{2}\right)$ increases continuously as $\mu_{2}$ increases. Therefore, the statement (i) is proven.

To know how $x_{\tilde{A}}^{d}\left(\mu_{1}, \mu_{2}\right)$ and $x_{\tilde{B}}^{d}\left(\mu_{1}, \mu_{2}\right)$ continuously depend on $\mu_{2}$, let $\left(x, \tilde{y}_{\mu_{1}, \mu_{2}}^{+}(x)\right)$ and $\left(x, \tilde{y}_{\mu_{1}, \mu_{2}}^{-}(x)\right)$ denote the points on $\gamma_{\mu_{1}, \mu_{2}}^{+}(d, 0)$ and $\gamma_{\mu_{1}, \mu_{2}}^{-}(d, 0)$ respectively. Then

$$
\tilde{y}_{\mu_{1}, \mu_{2}}^{+}(d)=0, \quad \tilde{y}_{\mu_{1}, \mu_{2}}^{-}(d)=0, \quad y_{\mu_{1}, \mu_{2}}^{+}\left(x_{\tilde{A}}^{d}\left(\mu_{1}, \mu_{2}\right)\right)=0 \quad \text { and } \quad y_{\mu_{1}, \mu_{2}}^{-}\left(x_{\tilde{B}}^{d}\left(\mu_{1}, \mu_{2}\right)\right)=0 .
$$

Let $\tilde{z}_{\mu_{1}, \mu_{2}}^{+}(x)=\tilde{y}_{\mu_{1}, \mu_{2}+\epsilon}^{+}(x)-\tilde{y}_{\mu_{1}, \mu_{2}}^{+}(x)$ for $d \leq x \leq \min \left\{x_{\tilde{A}}^{d}\left(\mu_{1}, \mu_{2}\right), x_{\tilde{A}}^{d}\left(\mu_{1}, \mu_{2}+\epsilon\right)\right\}$ be the vertical distance between $\gamma_{\mu_{1}, \mu_{2}+\epsilon}^{+}(d, 0)$ and $\gamma_{\mu_{1}, \mu_{2}}^{+}(d, 0)$, where $x_{\tilde{A}}^{d}\left(\mu_{1}, \mu_{2}+\epsilon\right)$ is the first intersection point of $\gamma_{\mu_{1}, \mu_{2}+\epsilon}^{+}(d, 0)$ and the positive $x$-axis and $|\epsilon|$ is sufficiently small. One can check that

$$
\begin{aligned}
\tilde{z}_{\mu_{1}, \mu_{2}}^{+}(x) & =\left.\left\{\tilde{y}_{\mu_{1}, \mu_{2}+\epsilon}^{+}(s)-\tilde{y}_{\mu_{1}, \mu_{2}}^{+}(s)\right\}\right|_{d} ^{x} \\
& =\int_{d}^{x}\left\{\left(\frac{\mu_{1} s-s^{3}}{\tilde{y}_{\mu_{1}, \mu_{2}+\epsilon}^{+}(s)}-\left(\mu_{2}+\epsilon\right)-\left|\tilde{y}_{\mu_{1}, \mu_{2}+\epsilon}^{+}(s)\right|\right)-\left(\frac{\mu_{1} s-s^{3}}{\tilde{y}_{\mu_{1}, \mu_{2}}^{+}(s)}-\mu_{2}-\left|\tilde{y}_{\mu_{1}, \mu_{2}}^{+}(s)\right|\right)\right\} d s \\
& =\tilde{H}_{1}(x)+\tilde{H}_{2}(x),
\end{aligned}
$$

where

$$
\tilde{H}_{1}(x)=-\epsilon(x-d), \quad \tilde{H}_{2}(x)=\int_{d}^{x} \tilde{z}_{\mu_{1}, \mu_{2}}^{+}(s) \tilde{H}_{3}(s) d s \quad \text { and } \quad \tilde{H}_{3}(x)=\frac{-\mu_{1} x+x^{3}}{\tilde{y}_{\mu_{1}, \mu_{2}+\epsilon}^{+}(x) \tilde{y}_{\mu_{1}, \mu_{2}}^{+}(x)}-1 .
$$

Similar to the calculation of $z_{\mu_{1}, \mu_{2}}^{+}(x)$, we get

$$
\tilde{z}_{\mu_{1}, \mu_{2}}^{+}(x)=-\epsilon \int_{d}^{x} \exp \left\{\int_{\tau}^{x} \tilde{H}_{3}(\eta) d \eta\right\} d \tau<0(\text { resp. }>0)
$$

if $\epsilon>0$ (resp. $<0)$. Then $x_{\tilde{A}}^{d}\left(\mu_{1}, \mu_{2}+\epsilon\right)<x_{\tilde{A}}^{d}\left(\mu_{1}, \mu_{2}\right)$ and $\lim _{\epsilon \rightarrow 0} x_{\tilde{A}}^{d}\left(\mu_{1}, \mu_{2}+\epsilon\right)=x_{\tilde{A}}^{d}\left(\mu_{1}, \mu_{2}\right)$. Moreover, $x_{\tilde{A}}^{d}\left(\mu_{1}, \mu_{2}\right)$ decreases continuously as $\mu_{2}$ increases.

Let $\tilde{z}_{\mu_{1}, \mu_{2}}^{-}(x)=\tilde{y}_{\mu_{1}, \mu_{2}+\epsilon}^{-}(x)-\tilde{y}_{\mu_{1}, \mu_{2}}^{-}(x)$ for $d \leq x \leq \min \left\{x_{\tilde{B}}^{d}\left(\mu_{1}, \mu_{2}\right), x_{\tilde{B}}^{d}\left(\mu_{1}, \mu_{2}+\epsilon\right)\right\}$ be the vertical distance between $\gamma_{\mu_{1}, \mu_{2}+\epsilon}^{-}(d, 0)$ and $\gamma_{\mu_{1}, \mu_{2}}^{-}(d, 0)$, where $x_{\tilde{B}}^{d}\left(\mu_{1}, \mu_{2}+\epsilon\right)$ is the first intersection point 
of $\gamma_{\mu_{1}, \mu_{2}+\epsilon}^{-}(d, 0)$ and the positive $x$-axis and $|\epsilon|$ is sufficiently small. Then

$$
\tilde{z}_{\mu_{1}, \mu_{2}}^{-}(x)=-\epsilon \int_{d}^{x} \exp \left\{\int_{\tau}^{x} \bar{H}_{3}(\eta) d \eta\right\} d \tau<0(\text { resp. }>0), \text { if } \epsilon>0(\text { resp. }<0),
$$

where $\bar{H}_{3}(x)=\left(-\mu_{1} x+x^{3}\right) /\left(\tilde{y}_{\mu_{1}, \mu_{2}+\epsilon}^{-}(x) \tilde{y}_{\mu_{1}, \mu_{2}}^{-}(x)\right)+1$. Moreover, $x_{\tilde{B}}^{d}\left(\mu_{1}, \mu_{2}+\epsilon\right)>x_{\tilde{B}}^{d}\left(\mu_{1}, \mu_{2}\right)$ and $\lim _{\epsilon \rightarrow 0} x_{\tilde{B}}^{d}\left(\mu_{1}, \mu_{2}+\epsilon\right)=x_{\tilde{B}}^{d}\left(\mu_{1}, \mu_{2}\right)$. Hence, $x_{\tilde{B}}^{d}\left(\mu_{1}, \mu_{2}\right)$ increases continuously as $\mu_{2}$ increases. Therefore, the statement (ii) is proven.

By Lemmas 3.3 and 3.4, we can get the nonexistence of small limit cycles and homoclinic loops when (c3) holds.

Lemma 3.5. When (c3) holds, there are neither small limit cycles nor homoclinic loops for system $(1.2 \mathrm{~b})$.

Proof. Since system $(1.2 \mathrm{~b})$ is symmetric about $E_{0}$ and $E_{0}$ is a saddle, it suffices to prove that there are no small limit cycles and no homoclinic loops surrounding $E_{r}$.

Firstly, we claim that any small limit cycle surrounding $E_{r}$ must lie in the region $|y|<-\mu_{2}$ when $\mu_{1}>0$ and $\mu_{2}=-2\left(12 \mu_{1}^{3}\right)^{1 / 4} / 3$. In fact, the graph of the horizontal isocline of system $(1.2 \mathrm{~b})$ is shown in Fig. 7 (b). Denote the segment of the horizontal isocline connecting $E_{0}$ and the point $A:\left(\sqrt{\mu_{1}}, \mu_{2}\right)$ by $\widehat{E_{0} A}$, as shown in Fig. 10. Notice that

$$
\left.\dot{x}\right|_{\widehat{E_{0} A}}=y<0,\left.\quad \dot{y}\right|_{\widehat{E_{0} A}}=0
$$

and

$$
\left.\dot{x}\right|_{x>\sqrt{\mu_{1}}, y=\mu_{2}}=y<0,\left.\quad \dot{y}\right|_{x>\sqrt{\mu_{1}}, y=\mu_{2}}=\mu_{1} x-x^{3}<0 .
$$

Then passing through a point in the fourth quadrant and below the line $y=\mu_{2}$ means that the limit cycle cannot be a small one.

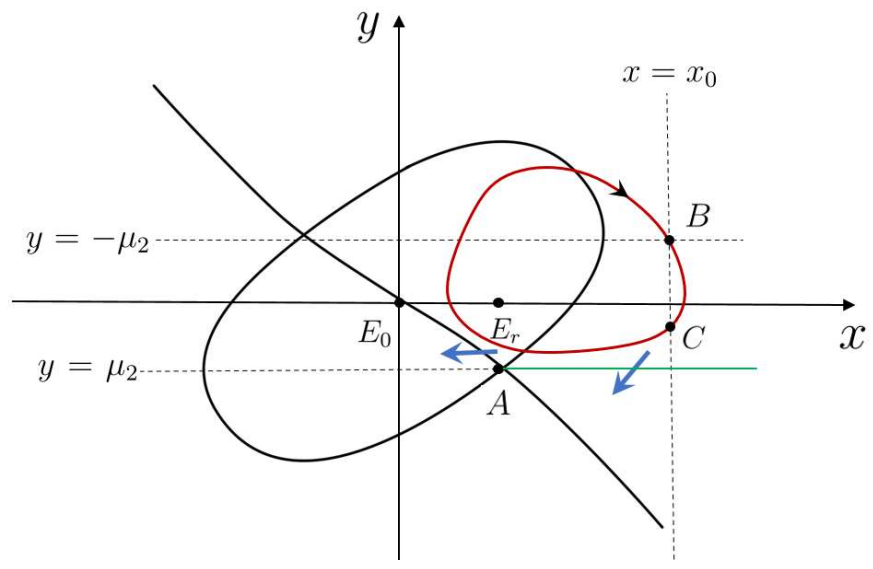

Fig. 10: The hypothetical limit cycle.

Assume that the peak point of a small limit cycle surrounding $E_{r}$ is above $y=-\mu_{2}$. The limit cycle will fall below $y=-\mu_{2}$ and move right down in the first quadrant, then move left and stay 
above $y=\mu_{2}$ in the fourth quadrant. Denote the rightmost intersection point of this small limit cycle and $y=-\mu_{2}$ by $B:\left(x_{0},-\mu_{2}\right)$, as shown in Fig. 10. Clearly, the limit cycle will intersect $x=x_{0}$ again at $C:\left(x_{0}, y_{0}\right)$, where $\mu_{2}<y_{0}<0$. Let

$$
\mathcal{E}(x, y):=\frac{y^{2}}{2}+\frac{x^{4}}{4}-\frac{\mu_{1} x^{2}}{2}
$$

which implies that

$$
\left.\frac{d \mathcal{E}}{d t}\right|_{(1.2 \mathrm{~b})}=-\left(\mu_{2}+|y|\right) y^{2}
$$

On the one hand, one can check that $\mathcal{E}\left(x_{0},-\mu_{2}\right)>\mathcal{E}\left(x_{0}, y_{0}\right)$ from $\mu_{2}<y_{0}<0$. On the other hand, the orbit segment $\widehat{B C}$ lies in the region $|y|<-\mu_{2}$. It follows from (3.7) that $d \mathcal{E} /\left.d t\right|_{\widehat{B C}}>0$, implying $\mathcal{E}\left(x_{0},-\mu_{2}\right)<\mathcal{E}\left(x_{0}, y_{0}\right)$. This is a contradiction.

Secondly, we prove that there are no small limit cycles surrounding $E_{r}$ when $\mu_{1}>0$ and $\mu_{2} \leq-2\left(12 \mu_{1}^{3}\right)^{1 / 4} / 3$. Since any small limit cycle surrounding $E_{r}$ lies in the region $|y|<-\mu_{2}$ when $\mu_{1}>0$ and $\mu_{2}=-2\left(12 \mu_{1}^{3}\right)^{1 / 4} / 3$, it follows from (3.7) that $d \mathcal{E} / d t>0$. That means the nonexistence of limit cycles for $\mu_{1}>0$ and $\mu_{2}=-2\left(12 \mu_{1}^{3}\right)^{1 / 4} / 3$. Thus,

$$
x_{\tilde{A}}^{d}\left(\mu_{1},-2\left(12 \mu_{1}^{3}\right)^{1 / 4} / 3\right)-x_{\tilde{B}}^{d}\left(\mu_{1},-2\left(12 \mu_{1}^{3}\right)^{1 / 4} / 3\right) \neq 0, \quad \forall 0<d<\sqrt{\mu_{1}} .
$$

Moreover, we claim that

$$
x_{\tilde{A}}^{d}\left(\mu_{1},-2\left(12 \mu_{1}^{3}\right)^{1 / 4} / 3\right)-x_{\tilde{B}}^{d}\left(\mu_{1},-2\left(12 \mu_{1}^{3}\right)^{1 / 4} / 3\right)>0, \quad \forall 0<d<\sqrt{\mu_{1}} .
$$

In fact if $x_{\tilde{A}}^{d_{*}}\left(\mu_{1},-2\left(12 \mu_{1}^{3}\right)^{1 / 4} / 3\right)-x_{\tilde{B}}^{d_{*}}\left(\mu_{1},-2\left(12 \mu_{1}^{3}\right)^{1 / 4} / 3\right)<0$ for some $d_{*} \in\left(0, \sqrt{\mu_{1}}\right)$, an annular region, whose $\omega$-limit set lies in itself, can be constructed because $E_{r}$ is unstable. By PoincaréBendixson Theorem, at least one small limit cycle surrounding $E_{r}$ exists.

From Lemma 3.4, $x_{\tilde{A}}^{d}\left(\mu_{1}, \mu_{2}\right)$ increases and $x_{\tilde{B}}^{d}\left(\mu_{1}, \mu_{2}\right)$ decreases as $\mu_{2}$ decreases. It follows from (3.8) that $x_{\tilde{A}}^{d}\left(\mu_{1}, \mu_{2}\right)-x_{\tilde{B}}^{d}\left(\mu_{1}, \mu_{2}\right)>0$ for any $0<d<\sqrt{\mu_{1}}$ when $\mu_{1}>0$ and $\mu_{2}<-2\left(12 \mu_{1}^{3}\right)^{1 / 4} / 3$. Then there are no limit cycles surrounding $E_{r}$ when $\mu_{1}>0$ and $\mu_{2}<-2\left(12 \mu_{1}^{3}\right)^{1 / 4} / 3$.

Thirdly, we prove that there are no homoclinic loops surrounding $E_{r}$ when $\mu_{1}>0$ and $\mu_{2} \leq$ $-2\left(12 \mu_{1}^{3}\right)^{1 / 4} / 3$. Notice that $\operatorname{tr} J_{0}=-\mu_{2}>0$, where $J_{0}$ is the Jacobian matrix (2.1). Due to [7, Theorem 3.3], the homoclinic loop of $E_{0}$ is asymptotically unstable if it exists. By Lemma 2.1, $E_{r}$ is unstable when $\mu_{1}>0$ and $\mu_{2} \leq-2\left(12 \mu_{1}^{3}\right)^{1 / 4} / 3$. Then the existence of homoclinic loops will lead to the existence of at least one small limit cycle surrounding $E_{r}$, which contradicts the conclusion of the second step.

To consider the number of large limit cycles for (c3), we investigate the relation of the divergence integrals of two hypothetic large limit cycles in the following lemma, which gives the monotonicity of the divergence integrals and also can be applied for the case (c4).

Lemma 3.6. When $\mu_{1}>0$ and $\mu_{2}<0$, if there are at least two large limit cycles for system (1.2b), the following assertion is true:

$$
\oint_{\Gamma_{1}} \operatorname{div}\left(y, \mu_{1} x-x^{3}-\left(\mu_{2}+|y|\right) y\right) d t>\oint_{\Gamma_{2}} \operatorname{div}\left(y, \mu_{1} x-x^{3}-\left(\mu_{2}+|y|\right) y\right) d t
$$

where $\Gamma_{1}, \Gamma_{2}$ are large limit cycles and $\Gamma_{1}$ lies in the region enclosed by $\Gamma_{2}$. 


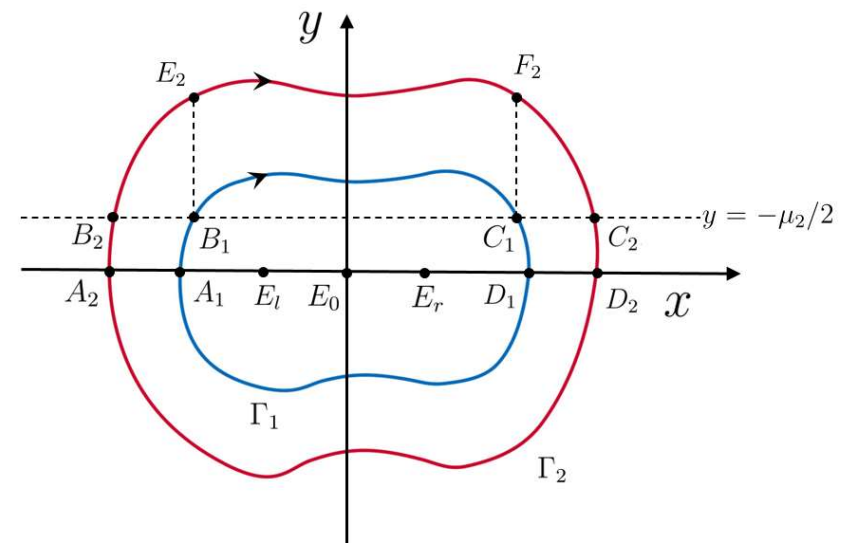

Fig. 11: Two large limit cycles.

Proof. Notice that the derivative of energy function $\mathcal{E}(x, y)$ defined in (3.6) with respect to $t$ is (3.7). Then any limit cycle can not lie in the region $|y|<-\mu_{2}$. For $i=1,2$, assume that $\Gamma_{i}$ crosses the $x$-axis and $y=-\mu_{2} / 2$ at $A_{i}, B_{i}, C_{i}$ and $D_{i}$ successively, as shown in Fig. 11. Passing through $B_{1}$ and $C_{1}$ respectively, lines perpendicular to the $x$-axis cross $\Gamma_{2}$ at two points, denote by $E_{2}$ and $F_{2}$. Let $x_{B_{1}}$ and $x_{C_{1}}$ be the abscissas of $B_{1}$ and $C_{1}$.

We claim that $x_{B_{1}}<-\sqrt{\mu_{1}}$ and $x_{C_{1}}>\sqrt{\mu_{1}}$. Since $\Gamma_{1}$ can not lie in the region $|y|<-\mu_{2}$, we denote the leftmost intersection point of $\Gamma$ and $y=-\mu_{2}$ by $P_{1}$, and the rightmost intersection point by $P_{2}$. Let $P_{3}$ and $P_{4}$ be the points $\left(-\sqrt{\mu_{1}},-\mu_{2}\right)$ and $\left(\sqrt{\mu_{1}},-\mu_{2}\right)$. We can prove $x_{B_{1}}<-\sqrt{\mu_{1}}$ and $x_{C_{1}}>\sqrt{\mu_{1}}$ by showing that $P_{1}$ is on the left side of $P_{3}$ and $P_{2}$ is on the right side of $P_{4}$.

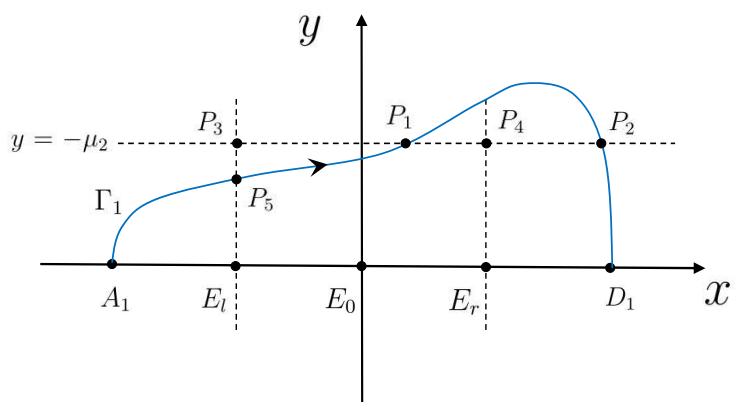

(a) $P_{1}$ is between $P_{3}$ and $P_{4}$

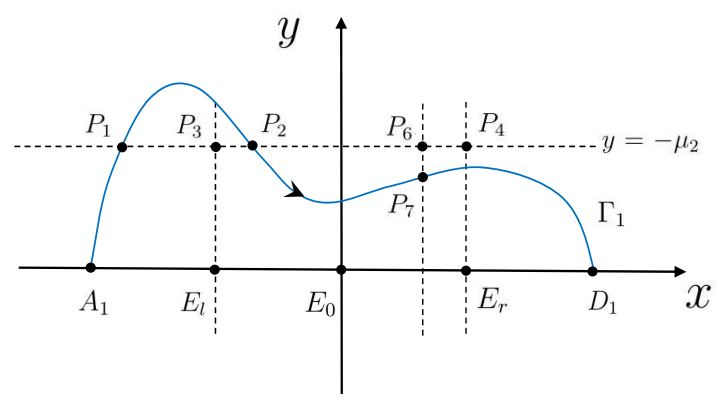

(b) $P_{2}$ is between $P_{3}$ and $P_{4}$

Fig. 12: The points lie in large limit cycle $\Gamma_{1}$.

From the graph of the horizontal isocline of system (1.2b), as shown in Fig. 6, $P_{1}$ can not be on the right side of $P_{4}$ and $P_{2}$ can not be on the left side of $P_{3}$. If $P_{1}$ is between $P_{3}$ and $P_{4}$, as shown in Fig. 12 (a), $\Gamma_{1}$ will cross $x=-\sqrt{\mu_{1}}$ at a point below $P_{3}$, denoted by $P_{5}$. From the expression of $d \mathcal{E} / d t$, as shown in $(3.7)$, we get $\mathcal{E}\left(A_{1}\right)<\mathcal{E}\left(P_{5}\right)$ and $\mathcal{E}\left(P_{2}\right)<\mathcal{E}\left(D_{1}\right)$. From (3.6) one can calculate $\mathcal{E}\left(P_{5}\right)<\mathcal{E}\left(P_{4}\right)<\mathcal{E}\left(P_{2}\right)$ and $\mathcal{E}\left(A_{1}\right)=\mathcal{E}\left(D_{1}\right)$. That implies a contradiction. Hence, $P_{1}$ lies on the left side of $P_{3}$. 
If $P_{2}$ is between $P_{3}$ and $P_{4}$, from the graph of the horizontal isocline (see Fig. 6), $P_{2}$ must be in the second quadrant. Denote the symmetric point of $P_{2}$ about the $y$-axis by $P_{6}$. Let $P_{7}$ be the point on $\Gamma_{1}$ such that the line connecting $P_{6}$ and $P_{7}$ is perpendicular to the $x$-axis. By the derivative of $\mathcal{E}(x, y)$, as shown in $(3.7)$, we get $\mathcal{E}\left(P_{2}\right)<\mathcal{E}\left(P_{7}\right)$. From (3.6), we calculate $\mathcal{E}\left(P_{2}\right)=\mathcal{E}\left(P_{6}\right)$ and $\mathcal{E}\left(P_{7}\right)<\mathcal{E}\left(P_{6}\right)$. That is a contradiction, indicating that $P_{2}$ lies on the right side of $P_{4}$.

Therefore, on the arc $\widehat{A_{i} B_{i}}$ and on the arc $\widehat{C_{i} D_{i}}, d y / d t$ is signed and $|x|>\sqrt{\mu_{1}}$. On the one hand, the arc $\widehat{A_{i} B_{i}}$ can be regarded as the graph of the function $x=x_{i}(y)$, where $0 \leq y \leq-\mu_{2} / 2$ and $i=1,2$. Thus,

$$
\begin{aligned}
& \int_{\widehat{A_{1} B_{1}}}\left(-\mu_{2}-2 y\right) d t-\int_{\widehat{A_{2} B_{2}}}\left(-\mu_{2}-2 y\right) d t \\
& =\int_{0}^{-\mu_{2} / 2} \frac{-\mu_{2}-2 y}{\mu_{1} x_{1}(y)-\left(x_{1}(y)\right)^{3}-\left(\mu_{2}+|y|\right) y} d y-\int_{0}^{-\mu_{2} / 2} \frac{-\mu_{2}-2 y}{\mu_{1} x_{2}(y)-\left(x_{2}(y)\right)^{3}-\left(\mu_{2}+|y|\right) y} d y \\
& =\int_{0}^{-\mu_{2} / 2} \frac{\left(-\mu_{2}-2 y\right)\left(x_{2}(y)-x_{1}(y)\right)\left(\mu_{1}-\left(x_{1}(y)\right)^{2}-x_{1}(y) x_{2}(y)-\left(x_{2}(y)\right)^{2}\right)}{\left(\mu_{1} x_{1}(y)-\left(x_{1}(y)\right)^{3}-\left(\mu_{2}+|y|\right) y\right)\left(\mu_{1} x_{2}(y)-\left(x_{2}(y)\right)^{3}-\left(\mu_{2}+|y|\right) y\right)}>0 .
\end{aligned}
$$

Similarly,

$$
\int_{\widehat{C_{1} D_{1}}}\left(-\mu_{2}-2 y\right) d t-\int_{\widehat{C_{2} D_{2}}}\left(-\mu_{2}-2 y\right) d t>0
$$

On the other hand, the arcs $\widehat{B_{1} C_{1}}$ and $\widehat{E_{2} F_{2}}$ can be regarded as graphs of functions $y=y_{1}(x)$ and $y=y_{2}(x)$ respectively, where $x_{B_{1}} \leq x \leq x_{C_{1}}$. Then

$$
\begin{aligned}
\int_{\widehat{B_{1} C_{1}}}\left(-\mu_{2}-2 y\right) d t-\int_{\widehat{E_{2} F_{2}}}\left(-\mu_{2}-2 y\right) d t & =\int_{x_{B_{1}}}^{x_{C_{1}}} \frac{-\mu_{2}-2 y_{1}(x)}{y_{1}(x)} d y-\int_{x_{B_{1}}}^{x_{C_{1}}} \frac{-\mu_{2}-2 y_{2}(x)}{y_{2}(x)} d y \\
& =\int_{x_{B_{1}}}^{x_{C_{1}}} \frac{-\mu_{2}\left(y_{2}(x)-y_{1}(x)\right)}{y_{1}(x) y_{2}(x)}>0 .
\end{aligned}
$$

Since $-\mu_{2}-2 y<0$ on the arcs $\widehat{B_{2} E_{2}}$ and $\widehat{F_{2} C_{2}}$, one can check that

$$
\int_{\widehat{B_{2} E_{2}}}\left(-\mu_{2}-2 y\right) d t<0, \quad \int_{\widehat{F_{2} C_{2}}}\left(-\mu_{2}-2 y\right) d t<0 .
$$

It follows from (3.9)-(3.12) that

$$
\begin{aligned}
& \oint_{\Gamma_{1}} \operatorname{div}\left(y, \mu_{1} x-x^{3}-\left(\mu_{2}+|y|\right) y\right) d t-\oint_{\Gamma_{2}} \operatorname{div}\left(y, \mu_{1} x-x^{3}-\left(\mu_{2}+|y|\right) y\right) d t \\
= & \oint_{\Gamma_{1}}\left(-\mu_{2}-2 y\right) d t-\oint_{\Gamma_{2}}\left(-\mu_{2}-2 y\right) d t \\
= & 2\left(\int_{\widehat{A_{1} D_{1}}}\left(-\mu_{2}-2 y\right) d t-\int_{\widehat{A_{2} D_{2}}}\left(-\mu_{2}-2 y\right) d t\right) \\
> & 0 .
\end{aligned}
$$

Therefore, the proof is finished.

From Lemma 3.6, when (c3) holds the uniqueness, stability and hyperbolicity of large limit cycles are given in the following lemma. Combining Lemma 3.5 and the following lemma, the qualitative properties of closed orbits of system (1.2b) with (c3) will be completely obtained.

Lemma 3.7. When (c3) holds, system (1.2b) exhibits a unique large limit cycle, which is stable and hyperbolic. 
Proof. We claim that $x_{A}^{0}\left(\mu_{1}, \mu_{2}\right)-x_{B}^{0}\left(\mu_{1}, \mu_{2}\right)>0$ when $\mu_{1}>0$ and $\mu_{2} \leq-2\left(12 \mu_{1}^{3}\right)^{1 / 4} / 3$. In fact, by Lemma 3.5 system (1.2b) exhibits no homoclinic loops, implying $x_{A}^{0}\left(\mu_{1}, \mu_{2}\right)-x_{B}^{0}\left(\mu_{1}, \mu_{2}\right) \neq 0$. If $x_{A}^{0}\left(\mu_{1}, \mu_{2}\right)-x_{B}^{0}\left(\mu_{1}, \mu_{2}\right)<0$, by the instability of $E_{r}$ and $\left.\dot{y}\right|_{x>\sqrt{\mu_{1}}, y>0}<0$, an annular region whose $\omega$-limit set lies in itself, can be constructed. By Poincaré-Bendixson Theorem, there is at least one small limit cycle surrounding $E_{r}$, which conflicts with Lemma 3.5.

By Lemma 2.3, both the equilibria at infinity are degenerate saddle-nodes and all orbits of system (1.2b) are positively bounded. Combining $x_{A}^{0}\left(\mu_{1}, \mu_{2}\right)-x_{B}^{0}\left(\mu_{1}, \mu_{2}\right)>0$, an annular region whose $\omega$-limit set lies in itself, can be constructed. By Poincaré-Bendixson Theorem, we get the existence of large limit cycles of system $(1.2 \mathrm{~b})$.

Denote by $\Gamma$ the innermost large limit cycle for $(1.2 \mathrm{~b})$. Since $x_{A}^{0}\left(\mu_{1}, \mu_{2}\right)-x_{B}^{0}\left(\mu_{1}, \mu_{2}\right)>0$ when $\mu_{1}>0$ and $\mu_{2} \leq-2\left(12 \mu_{1}^{3}\right)^{1 / 4} / 3$, we get that $\Gamma$ is internally stable. Then

$$
\oint_{\Gamma} \operatorname{div}\left(y, \mu_{1} x-x^{3}-\left(\mu_{2}+|y|\right) y\right) \leq 0 .
$$

If $\oint_{\Gamma} \operatorname{div}\left(y, \mu_{1} x-x^{3}-\left(\mu_{2}+|y|\right) y\right)=0, \Gamma$ is externally unstable. By Lemma 3.4 and [21, Theorem 3.4 of Chapter 3.4], at least two limit cycles will be bifurcated from $\Gamma$, including a stable inner limit cycle and an unstable outer one, when $\mu_{2}$ changes to a larger value, which contradicts the conclusion of Lemma 3.6. Thus,

$$
\oint_{\Gamma} \operatorname{div}\left(y, \mu_{1} x-x^{3}-\left(\mu_{2}+|y|\right) y\right)<0 .
$$

Moreover, $\Gamma$ is stable and hyperbolic by (3.13). Notice that any two adjacent closed orbits cannot stable simultaneously and $\Gamma$ is the innermost large limit cycle. It follows from Lemma 3.6 that $\Gamma$ is the unique large limit cycle for system $(1.2 \mathrm{~b})$.

When (c4) holds, the number of large limit cycles is different for different parameters. In the following lemma, we first give an upper bound on the number of large limit cycles and discuss the stabilities of large limit cycles.

Lemma 3.8. When (c4) holds, there are at most two large limit cycles for system (1.2b), and

(a) the inner limit cycle is unstable and the outer one is stable if there are two limit cycles;

(b) the limit cycle is stable or semi-stale (internally unstable and externally stable) if there is a unique limit cycle.

Proof. Firstly, we prove that there are at most two large limit cycles for system (1.2b) when $\mu_{1}>0$ and $-2\left(12 \mu_{1}^{3}\right)^{1 / 4} / 3<\mu_{2}<0$. Assume that system $(1.2 \mathrm{~b})$ has at least three large limit cycles. Denote the third outer large limit cycle, seconde outer one and outermost one by $\Gamma_{1}, \Gamma_{2}$ and $\Gamma_{3}$ respectively. By Lemma 2.3, the outermost large limit cycle $\Gamma_{3}$ is externally stable. Then $\oint_{\Gamma_{3}} \operatorname{div}\left(y, \mu_{1} x-x^{3}-\left(\mu_{2}+|y|\right) y\right) \leq 0$. Since any two closed orbits with the same stability cannot be adjacent to each other, by Lemma 3.6

$$
\oint_{\Gamma_{1}} \operatorname{div}\left(y, \mu_{1} x-x^{3}-\left(\mu_{2}+|y|\right) y\right)>0, \quad \oint_{\Gamma_{2}} \operatorname{div}\left(\left(y, \mu_{1} x-x^{3}-\left(\mu_{2}+|y|\right) y\right)\right)=0
$$


and

$$
\oint_{\Gamma_{3}} \operatorname{div}\left(\left(y, \mu_{1} x-x^{3}-\left(\mu_{2}+|y|\right) y\right)\right)<0
$$

That means $\Gamma_{1}$ is unstable, $\Gamma_{3}$ is stable and $\Gamma_{2}$ is internally stable and externally unstable. By Lemma 3.4 and [21, Theorem 3.4 of Chapter 3.4], a stable inner limit cycle and an unstable outer one will be bifurcated from $\Gamma_{2}$ when $\mu_{2}$ varies to a larger value, which is contradictory with the conclusion of Lemma 3.6.

Secondly, we prove the statement (a). Assume that there are exactly two large limit cycles for system (1.2b), denoted by $\Gamma_{1}$ and $\Gamma_{2}$ from insider to outsider. By Lemma 2.3, $\Gamma_{2}$ is externally stable. If $\Gamma_{2}$ is internally unstable and externally stable,

$$
\oint_{\Gamma_{2}} \operatorname{div}\left(\left(y, \mu_{1} x-x^{3}-\left(\mu_{2}+|y|\right) y\right)\right)=0
$$

By Lemma 3.6,

$$
\oint_{\Gamma_{1}} \operatorname{div}\left(\left(y, \mu_{1} x-x^{3}-\left(\mu_{2}+|y|\right) y\right)\right)>0 .
$$

Thus, $\Gamma_{1}$ is unstable, which conflicts with the fact that $\Gamma_{1}$ is in the region enclosed by $\Gamma_{2}$ and they are adjacent to each other. Therefore, $\Gamma_{2}$ is stable.

The stability of $\Gamma_{2}$ implies that $\Gamma_{1}$ is externally unstable. If $\Gamma_{1}$ is externally unstable and internally unstable, by Lemma 3.4 and [21, Theorem 3.4 of Chapter 3.4], a stable inner limit cycle and an unstable outer one will be bifurcated from $\Gamma_{1}$. That contradicts the conclusion of Lemma 3.6. Then $\Gamma_{1}$ is unstable, yielding that the statement (a) is proven.

Thirdly, we prove the statement (b). Assume that there is a unique large limit cycle for system (1.2b), denoted by $\Gamma$. By Lemma $2.3, \Gamma$ is externally stable. Then $\Gamma$ is stable or semi-stale (internally unstable and externally stable), implying that the statement (b) is proven.

The following lemma gives a region where only stable large limit cycles may exist.

Lemma 3.9. When (c4) holds, system (1.2b) exhibits at most one large limit cycle in the region $\mathbb{R}^{2} \backslash\left\{(x, y):|x| \leq \sqrt{\mu_{1}},|y|<-\mu_{2} / 2\right\}$. Moreover, the large limit cycle is hyperbolic and stable if it exists.

Proof. Assume that there is a large limit cycle $\Gamma$ in the region $\mathbb{R}^{2} \backslash\left\{(x, y):|x| \leq \sqrt{\mu_{1}},|y|<-\mu_{2} / 2\right\}$ for system $(1.2 \mathrm{~b})$. We will prove the uniqueness, hyperbolicity and stability of $\Gamma$ by showing $\oint_{\Gamma} \operatorname{div}\left(y, \mu_{1} x-x^{3}-\left(\mu_{2}+|y|\right) y\right) d t<0$.

As in the proof of Lemma 3.6, $\Gamma$ will cross the $x$-axis, the lines $y=-\mu_{2} / 2$ and $y=-\mu_{2}$ successively. Denote the intersections points by $A, B, C, D, E$ and $F$, as shown in Fig. 13. Let $x_{C}$ and $x_{D}$ be the abscissas of $C$ and $D$. Then $x_{C}<-\sqrt{\mu_{1}}$ and $x_{D}>\sqrt{\mu_{1}}$.

Combining the graph of the horizontal isocline when $-2\left(12 \mu_{1}^{3}\right)^{1 / 4} / 3<\mu_{2}<0$ (see Fig. 6 (a)), the arc $\widehat{A B}$ can be regarded as the graph of the function $x=x_{1}(y), 0 \leq y \leq-\mu_{2} / 2$, and 


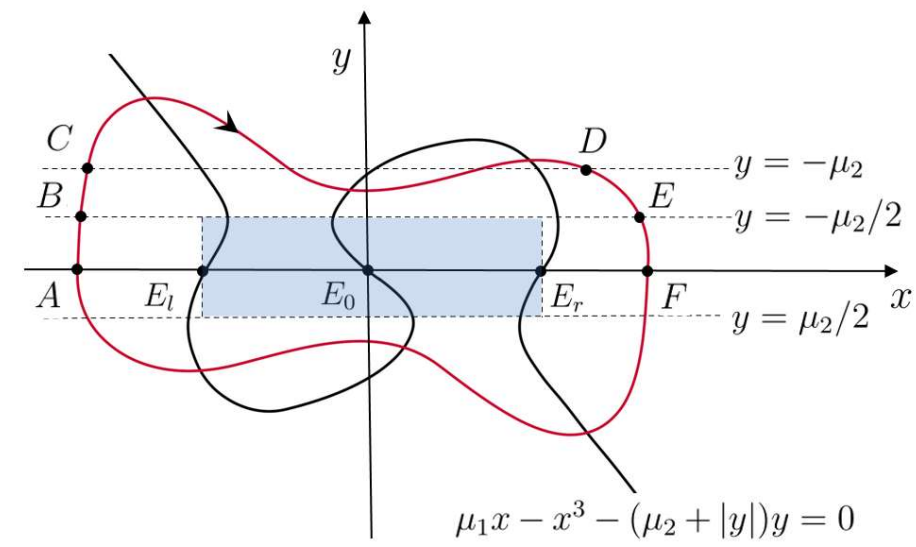

Fig. 13: A large limit cycle in the region $\mathbb{R}^{2} \backslash\left\{(x, y):|x| \leq \sqrt{\mu_{1}},|y|<-\mu_{2} / 2\right\}$.

the arc $\widehat{B C}$ can be seen as the graph of the function $x=x_{2}(y)$ for $-\mu_{2} / 2 \leq y \leq-\mu_{2}$. Clearly, $x_{1}\left(-\mu_{2} / 2\right)=x_{2}\left(-\mu_{2} / 2\right)$. Then

$$
\begin{aligned}
\int_{\widehat{A B} \cup \widehat{B C}}\left(-\mu_{2}-2 y\right) d t & =\int_{0}^{-\mu_{2} / 2} \frac{-\mu_{2}-2 y}{f_{1}(y)-\left(\mu_{2}+y\right) y} d y+\int_{-\mu_{2} / 2}^{-\mu_{2}} \frac{-\mu_{2}-2 y}{f_{2}(y)-\left(\mu_{2}+y\right) y} d y \\
& =\int_{0}^{-\mu_{2} / 2} \frac{-\mu_{2}-2 y}{f_{1}(y)-\left(\mu_{2}+y\right) y} d y+\int_{-\mu_{2} / 2}^{0} \frac{-\mu_{2}-2 s}{f_{2}\left(-\mu_{2}-s\right)-\left(\mu_{2}+s\right) s} d s \\
& =\int_{0}^{-\mu_{2} / 2} \frac{\left(-\mu_{2}-2 y\right)\left(f_{2}\left(-\mu_{2}-y\right)-f_{1}(y)\right)}{\left(f_{1}(y)-\left(\mu_{2}+y\right) y\right)\left(f_{2}\left(-\mu_{2}-y\right)-\left(\mu_{2}+y\right) y\right)} d y,
\end{aligned}
$$

where $f_{1}(y)=\mu_{1} x_{1}(y)-\left(x_{1}(y)\right)^{3}$ and $f_{2}(y)=\mu_{1} x_{2}(y)-\left(x_{2}(y)\right)^{3}$. On the one hand, from $\dot{x}=y$ we get that $x_{1}(y)<x_{1}\left(-\mu_{2} / 2\right)$ for $0 \leq y \leq-\mu_{2} / 2$ and $x_{2}(y)>x_{2}\left(-\mu_{2} / 2\right)$ for $-\mu_{2} / 2 \leq y \leq-\mu_{2}$, indicating that $x_{2}\left(-\mu_{2}-y\right)>x_{1}(y)$ for $0 \leq y \leq-\mu_{2} / 2$. On the other hand, $x_{C}=x_{2}\left(-\mu_{2}\right)<-\sqrt{\mu_{1}}$ means $x_{1}(y)<-\sqrt{\mu_{1}}$ and $x_{2}\left(-\mu_{2}-y\right)<-\sqrt{\mu_{1}}$ for $0 \leq y \leq-\mu_{2} / 2$. Thus,

$$
f_{2}\left(-\mu_{2}-y\right)-f_{1}(y)=\left(x_{2}\left(-\mu_{2}-y\right)-x_{1}(y)\right)\left(u_{1}-\left(x_{1}(y)\right)^{2}-x_{1}(y) x_{2}\left(-\mu_{2}-y\right)-\left(x_{2}\left(-\mu_{2}-y\right)\right)^{2}\right)<0 .
$$

It follows from (3.14) that we have

$$
\int_{\widehat{A B} \cup \widehat{B C}}\left(-\mu_{2}-2 y\right) d t<0
$$

Similarly,

$$
\int_{\widehat{D E} \cup \widehat{E F}}\left(-\mu_{2}-2 y\right) d t<0 .
$$

Notice that $\Gamma$ is in the region $\mathbb{R}^{2} \backslash\left\{(x, y):|x| \leq \sqrt{\mu_{1}},|y|<-\mu_{2} / 2\right\}$. One can check that $-\mu_{2}-2 y<0$ on the arc $\widehat{C D}$. Then

$$
\int_{\widehat{C D}}\left(-\mu_{2}-2 y\right) d t<0
$$

From (3.15), (3.16) and (3.17), we have

$$
\oint_{\Gamma} \operatorname{div}\left(y, \mu_{1} x-x^{3}-\left(\mu_{2}+|y|\right) y\right) d t=2 \int_{\widehat{A B} \cup \widehat{B C} \cup \widehat{C D} \cup \widehat{D E} \cup \widehat{E F}}\left(-\mu_{2}-2 y\right) d t<0 .
$$


By the transformation $(x, y, t) \rightarrow\left(\sqrt{\mu_{1}} x, \mu_{1} y, t / \sqrt{\mu_{1}}\right)$, system $(1.2 \mathrm{~b})$ can be reduced into

$$
\dot{x}=y, \quad \dot{y}=x-x^{3}-\frac{\mu_{2}}{\sqrt{\mu_{1}}} y-\sqrt{\mu_{1}}|y| y .
$$

Obviously, system (3.18) has the same topological structure as system (1.2b). Similarly to the discussions above Lemma 3.4, the positive orbits starting at $(x, y) \in\{(0, c): c \geq 0\} \cup\{(d, 0): 0 \leq$ $d<1\}$ and the negative orbits starting at $(x, y) \in\{(0,-c): c \geq 0\} \cup\{(d, 0): 0 \leq d<1\}$ both cross the positive $x$-axis. Denote the first intersections points by $\chi_{A}^{c}\left(\mu_{1}, \mu_{2}\right), \chi_{\tilde{A}}^{d}\left(\mu_{1}, \mu_{2}\right), \chi_{B}^{c}\left(\mu_{1}, \mu_{2}\right)$ and $\chi_{\tilde{B}}^{d}\left(\mu_{1}, \mu_{2}\right)$.

To show that the vector field of system (3.18) is rotated with respect to $\mu_{1}$ and $\mu_{2}$, we give the continuity and monotonicity of $\chi_{A}^{c}\left(\mu_{1}, \mu_{2}\right), \chi_{\tilde{A}}^{d}\left(\mu_{1}, \mu_{2}\right), \chi_{B}^{c}\left(\mu_{1}, \mu_{2}\right)$ and $\chi_{\tilde{B}}^{d}\left(\mu_{1}, \mu_{2}\right)$ in following theorem.

Lemma 3.10. Consider $\mu_{1}>0$ and $\mu_{2}<0$.

(i) For a fixed $\mu_{1}$, both $\chi_{A}^{c}\left(\mu_{1}, \mu_{2}\right)$ and $\chi_{\tilde{A}}^{d}\left(\mu_{1}, \mu_{2}\right)$ decreases continuously, and both $\chi_{B}^{c}\left(\mu_{1}, \mu_{2}\right)$ and $\chi_{\tilde{B}}^{d}\left(\mu_{1}, \mu_{2}\right)$ increases continuously as $\mu_{2}$ increases;

(ii) For a fixed $\mu_{2}$, both $\chi_{A}^{c}\left(\mu_{1}, \mu_{2}\right)$ and $\chi_{\tilde{A}}^{d}\left(\mu_{1}, \mu_{2}\right)$ decreases continuously, and both $\chi_{B}^{c}\left(\mu_{1}, \mu_{2}\right)$ and $\chi_{\tilde{B}}^{d}\left(\mu_{1}, \mu_{2}\right)$ increases continuously as $\mu_{1}$ increases,

where $c \geq 0$ and $0 \leq d<1$.

Proof. The process and method in this proof are almost same as Lemma 3.4. As long as the vertical distance between the orbits of system (3.18) with $\left(\mu_{1}, \mu_{2}\right)$ and system $(3.18)$ with $\left(\mu_{1}, \mu_{2}+\epsilon\right)$ (resp. $\left.\left(\mu_{1}+\delta, \mu_{2}\right)\right)$ passing through a same point is given, the statement (i) (resp. (ii)) can be obtained.

Denote points on the positive orbit passing through $(0, c)$ and negative orbit passing through $(0,-c)$ of system $(3.18)$ with $\left(\mu_{1}, \mu_{2}\right)$ by $\left(x, y_{\mu_{1}, \mu_{2}}^{+}(x)\right)$ and $\left(x, y_{\mu_{1}, \mu_{2}}^{-}(x)\right)$ respectively. Similar to calculate $z_{\mu_{1}, \mu_{2}}^{+}(x)$ and $z_{\mu_{1}, \mu_{2}}^{-}(x)$ in (3.4) and (3.5), the vertical distances $y_{\mu_{1}, \mu_{2}+\epsilon}^{+}(x)-y_{\mu_{1}, \mu_{2}}^{+}(x)$ and $y_{\mu_{1}, \mu_{2}+\epsilon}^{-}(x)-y_{\mu_{1}, \mu_{2}}^{-}(x)$ are

$$
y_{\mu_{1}, \mu_{2}+\epsilon}^{+}(x)-y_{\mu_{1}, \mu_{2}}^{+}(x)=-\frac{\epsilon}{\sqrt{\mu_{1}}} \int_{0}^{x} \exp \left\{\int_{\tau}^{x} K_{1}(\eta) d \eta\right\} d \tau
$$

for $0 \leq x \leq \min \left\{\chi_{A}^{c}\left(\mu_{1}, \mu_{2}\right), \chi_{A}^{c}\left(\mu_{1}, \mu_{2}+\epsilon\right)\right\}$ and

$$
y_{\mu_{1}, \mu_{2}+\epsilon}^{-}(x)-y_{\mu_{1}, \mu_{2}}^{-}(x)=-\frac{\epsilon}{\sqrt{\mu_{1}}} \int_{0}^{x} \exp \left\{\int_{\tau}^{x} K_{2}(\eta) d \eta\right\} d \tau
$$

for $0 \leq x \leq \min \left\{\chi_{B}^{c}\left(\mu_{1}, \mu_{2}\right), \chi_{B}^{c}\left(\mu_{1}, \mu_{2}+\epsilon\right)\right\}$, where

$$
K_{1}(x)=\frac{-x+x^{3}}{y_{\mu_{1}, \mu_{2}+\epsilon}^{+}(x) y_{\mu_{1}, \mu_{2}}^{+}(x)}-\sqrt{\mu_{1}}, \quad K_{2}(x)=\frac{-x+x^{3}}{y_{\mu_{1}, \mu_{2}+\epsilon}^{-}(x) y_{\mu_{1}, \mu_{2}}^{-}(x)}+\sqrt{\mu_{1}} .
$$

Then

$$
\left.y_{\mu_{1}, \mu_{2}+\epsilon}^{ \pm}(x)-y_{\mu_{1}, \mu_{2}}^{ \pm}(x)<0(\text { resp. }>0) \text {, if } \epsilon>0 \text { (resp. }<0\right) .
$$


Thus, $\chi_{A}^{c}\left(\mu_{1}, \mu_{2}\right)$ decreases continuously and $\chi_{B}^{c}\left(\mu_{1}, \mu_{2}\right)$ increases continuously as $\mu_{2}$ increases. In the same way, we can also calculate the vertical distances

$$
\begin{aligned}
& y_{\mu_{1}+\delta, \mu_{2}}^{+}(x)-y_{\mu_{1}, \mu_{2}}^{+}(x) \\
& =\left(\sqrt{\mu_{1}+\delta}-\sqrt{\mu_{1}}\right) \int_{0}^{x}\left(\frac{\mu_{2}}{\sqrt{\mu_{1}\left(\mu_{1}+\delta\right)}}-y_{\mu_{1}+\delta, \mu_{2}}^{+}(\tau)\right) \exp \left\{\int_{\tau}^{x} K_{3}(\eta) d \eta\right\} d \tau
\end{aligned}
$$

for $0 \leq x \leq \min \left\{\chi_{A}\left(\mu_{1}, \mu_{2}\right), \chi_{A}\left(\mu_{1}+\delta, \mu_{2}\right)\right\}$ and

$$
\begin{aligned}
& y_{\mu_{1}+\delta, \mu_{2}}^{-}(x)-y_{\mu_{1}, \mu_{2}}^{-}(x) \\
& \quad=\left(\sqrt{\mu_{1}+\delta}-\sqrt{\mu_{1}}\right) \int_{0}^{x}\left(\frac{\mu_{2}}{\sqrt{\mu_{1}\left(\mu_{1}+\delta\right)}}+y_{\mu_{1}+\delta, \mu_{2}}^{-}(\tau)\right) \exp \left\{\int_{\tau}^{x} K_{4}(\eta) d \eta\right\} d \tau
\end{aligned}
$$

for $0 \leq x \leq \min \left\{\chi_{B}\left(\mu_{1}, \mu_{2}\right), \chi_{B}\left(\mu_{1}+\delta, \mu_{2}\right)\right\}$, where

$$
K_{3}(x)=\frac{-x+x^{3}}{y_{\mu_{1}+\delta, \mu_{2}}^{+}(x) y_{\mu_{1}, \mu_{2}}^{+}(x)}-\sqrt{\mu_{1}}, \quad K_{4}(x)=\frac{-x+x^{3}}{y_{\mu_{1}+\delta, \mu_{2}}^{-}(x) y_{\mu_{1}, \mu_{2}}^{-}(x)}+\sqrt{\mu_{1}} .
$$

Then

$$
\left.\left.y_{\mu_{1}+\delta, \mu_{2}}^{ \pm}(x)-y_{\mu_{1}, \mu_{2}}^{ \pm}(x)<0 \text { (resp. }>0\right) \text {, if } \delta>0 \text { (resp. }<0\right) .
$$

Thus, $\chi_{A}^{c}\left(\mu_{1}, \mu_{2}\right)$ decreases continuously and $\chi_{B}^{c}\left(\mu_{1}, \mu_{2}\right)$ increases continuously as $\mu_{1}$ increases.

Denote points on the positive orbit and negative orbit passing through $(d, 0)$ of system (3.18) with $\left(\mu_{1}, \mu_{2}\right)$ by $\left(x, \tilde{y}_{\mu_{1}, \mu_{2}}^{+}(x)\right)$ and $\left(x, \tilde{y}_{\mu_{1}, \mu_{2}}^{-}(x)\right)$ respectively. The vertical distances $\tilde{y}_{\mu_{1}, \mu_{2}+\epsilon}^{+}(x)-$ $\tilde{y}_{\mu_{1}, \mu_{2}}^{+}(x), \tilde{y}_{\mu_{1}, \mu_{2}+\epsilon}^{-}(x)-\tilde{y}_{\mu_{1}, \mu_{2}}^{-}(x), \tilde{y}_{\mu_{1}+\delta, \mu_{2}}^{+}(x)-\tilde{y}_{\mu_{1}, \mu_{2}}^{+}(x)$ and $\tilde{y}_{\mu_{1}+\delta, \mu_{2}}^{-}(x)-\tilde{y}_{\mu_{1}, \mu_{2}}^{-}(x)$ have the similar expressions with (3.19), (3.20), (3.21) and (3.22) respectively except that the lower limit integration with respect to $\tau$ is $d$ rather than 0 .

As in Lemma 3.9 for large limit cycles, we also gives a region where only stable small limit cycles may exist.

Lemma 3.11. When (c4) holds, system (1.2b) exhibits at most one small limit cycle in the region $\left\{(x, y): x \geq \sqrt{\mu_{1} / 3}\right\}$. Moreover, the small limit cycle is hyperbolic and stable if it exists.

Proof. Assume that there is a small limit cycle $\gamma$ in the region $\left\{(x, y): x \geq \sqrt{\mu_{1} / 3}\right\}$ for system (1.2b). Clearly, $\gamma$ surrounds $E_{r}$. Combining the graph of the horizontal isoclinic of system (1.2b), as shown in Fig. 6 (a), $\gamma$ will intersect with $x=\sqrt{\mu_{1}}$ at two points, denoted by $M_{1}$ and $M_{2}$ from top to bottom. Let $M_{3}$ and $M_{4}$ be the points $\left(\sqrt{\mu_{1}},-\mu_{2}\right)$ and $\left(\sqrt{\mu_{1}}, \mu_{2}\right)$ respectively.

We claim that $M_{1}$ is above $M_{3}$ and $M_{2}$ is below $M_{4}$. From (3.6) and (3.7), the derivative of the energy function $\mathcal{E}(x, y)$ is positive in the region $|y|<-\mu_{2}$, implying that at least one of $M_{1}$ and $M_{2}$ falls outside of the line segment $\overline{M_{3} M_{4}}$. If $M_{1}$ is below $M_{3}$ and $M_{2}$ is below $M_{4}$, as shown in Fig. 14 (a), $\gamma$ will intersect with $y=\mu_{2}$ at a point on the left side of $M_{4}$, denoted by $M_{5}$. Since the orbit segment $\widehat{M_{5} M_{1}}$ is in the region $|y|<-\mu_{2}$, by $(3.7)$ we get $\mathcal{E}\left(M_{5}\right)<\mathcal{E}\left(M_{1}\right)$. One can calculate $\mathcal{E}\left(M_{1}\right)<\mathcal{E}\left(M_{3}\right)=\mathcal{E}\left(M_{4}\right)<\mathcal{E}\left(M_{5}\right)$ from (3.6), which is a contradiction. If $M_{1}$ is above 


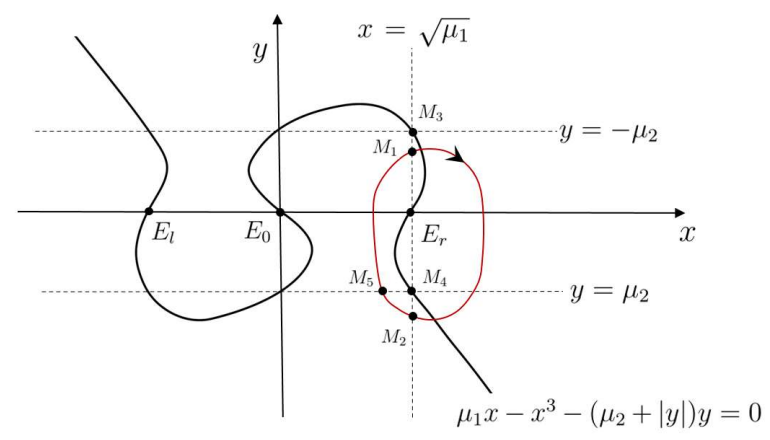

(a) $M_{1}$ is below $M_{3}$

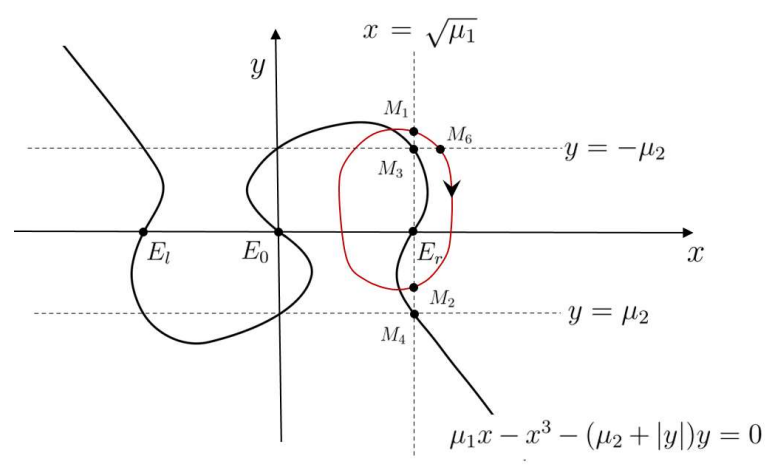

(b) $M_{2}$ is above $M_{4}$

Fig. 14: The positions of $\gamma$ in the hypothesis.

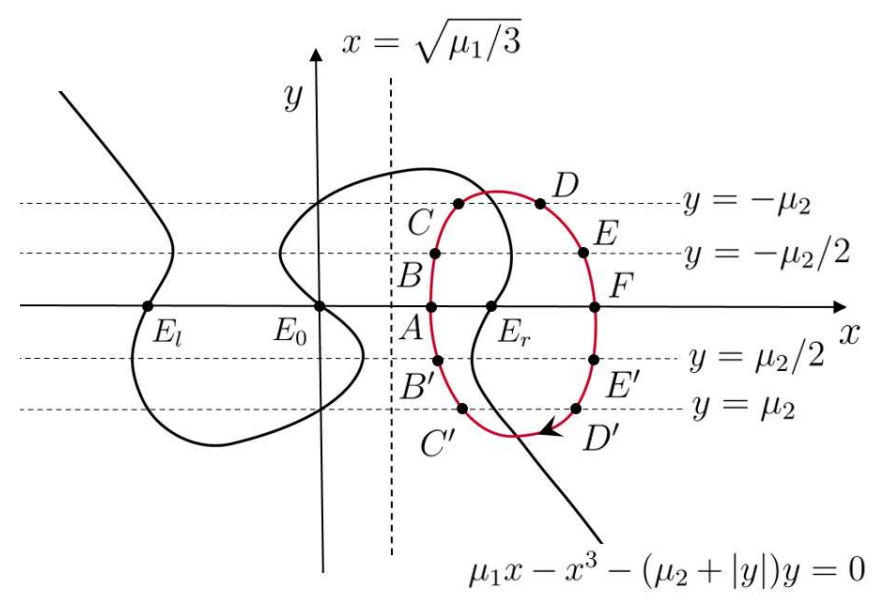

Fig. 15: A small limit cycle in the region $\left\{(x, y): x \geq \sqrt{\mu_{1} / 3}\right\}$.

$M_{3}$ and $M_{2}$ is above $M_{4}$, as shown in Fig. 14 (b), $\gamma$ will intersect with $y=-\mu_{2}$ at a point on the right side of $M_{3}$, denoted by $M_{6}$. Similarly, $\mathcal{E}\left(M_{6}\right)<\mathcal{E}\left(M_{2}\right)$ can be obtained from (3.7) and $\mathcal{E}\left(M_{6}\right)>\mathcal{E}\left(M_{3}\right)=\mathcal{E}\left(M_{4}\right)>\mathcal{E}\left(M_{2}\right)$ can be calculated from (3.6). This is a contradictory statement.

Denote the intersection points of $\gamma$ with the $x$-axis, the lines $y= \pm \mu_{2} / 2$ and $y= \pm \mu_{2}$ by $A$, $B, C, D, E, F, E^{\prime}, D^{\prime}, C^{\prime}, B^{\prime}$, as shown in Fig. 15. Since $\dot{y}>0$ along $\widehat{A C}$, let $x=x_{1}(y)$, $0 \leq y \leq-\mu_{2} / 2$ and $x=x_{2}(y),-\mu_{2} / 2 \leq y \leq-\mu_{2}$ be the functions of arcs $\widehat{A B}$ and $\widehat{B C}$. Then as same as calculation of (3.14), we can get

$$
\int_{\widehat{A B} \cup \widehat{B C}}\left(-\mu_{2}-2|y|\right) d t=\int_{0}^{-\mu_{2} / 2} \frac{\left(-\mu_{2}-2 y\right)\left(f_{2}\left(-\mu_{2}-y\right)-f_{1}(y)\right)}{\left.\left.\dot{y}\right|_{x=x_{1}(y)} \cdot \dot{y}\right|_{x=x_{2}\left(-\mu_{2}-y\right)}} d y,
$$

where $f_{1}$ and $f_{2}$ are defined under (3.14). Obviously, $x_{1}\left(-\mu_{2} / 2\right)=x_{2}\left(-\mu_{2} / 2\right)$. From $\dot{x}>0$ along $\widehat{A C}$, it is easy to see that $\sqrt{\mu_{1} / 3}<x_{1}(y)<x_{1}\left(-\mu_{2} / 2\right)$ for $0 \leq y \leq-\mu_{2} / 2$ and $x_{2}(y)>x_{2}\left(-\mu_{2} / 2\right)$ for $-\mu_{2} / 2 \leq y \leq-\mu_{2}$. Thus, $\sqrt{\mu_{1} / 3}<x_{1}(y)<x_{2}\left(-\mu_{2}-y\right)$, which implies $f_{2}\left(-\mu_{2}-y\right)-f_{1}(y)<0$ and

$$
\int_{\widehat{A B} \cup \widehat{B C}}\left(-\mu_{2}-2|y|\right) d t<0
$$


Similarly,

$$
\int_{\widehat{D E} \cup \widehat{E F}}\left(-\mu_{2}-2|y|\right) d t<0, \quad \int_{\widehat{F E^{\prime}} \widehat{\cup E^{\prime} D^{\prime}}}\left(-\mu_{2}-2|y|\right) d t<0
$$

and

$$
\int_{\widehat{C^{\prime} B^{\prime} \cup \widehat{B^{\prime} A}}}\left(-\mu_{2}-2|y|\right) d t<0 .
$$

Since $-\mu_{2}-2|y|>0$ along $\widehat{C D}$ and $\widehat{D^{\prime} C^{\prime}}$,

$$
\int_{\widehat{C D}}\left(-\mu_{2}-2|y|\right) d t<0, \quad \int_{\widehat{D^{\prime} C^{\prime}}}\left(-\mu_{2}-2|y|\right) d t<0 .
$$

From (3.23-3.26),

$$
\oint_{\gamma} \operatorname{div}\left(y, \mu_{1} x-x^{3}-\left(\mu_{2}+|y|\right) y\right) d t=\oint_{\gamma}\left(-\mu_{2}-2|y|\right)<0
$$

implying that $\gamma$ is stable, hyperbolic and is the unique limit cycle in the region $\{(x, y): x \geq$ $\left.\sqrt{\mu_{1} / 3}\right\}$.

For the limit cycles passing through the region $\left\{(x, y): 0<x<\sqrt{\mu_{1} / 3}\right\}$, the number is difficult to be gained theoretically. In section 6, numerical simulations shows that small limit cycles surrounding the same equilibrium are gluing together and cannot be distinguished. Based on the number of small limit cycles surrounding one equilibrium in focus case of smooth RayleighDuffing oscillator [6] and the numerical simulations of system (1.2b), we conjecture that there are at most two limit cycles surrounding $E_{r}$ for system (1.2b). All our later conclusions are based on the conjecture. If the conjecture is invalid, system (1.2b) will exhibit more complex dynamical behavior. But the closed orbits and bifurcation curves in the following conclusions remain while more limit cycles and bifurcation curves appear which are very close to the original ones.

Lemma 3.12. When (c4) holds, there are at most two small limit cycles surrounding $E_{r}$ for system $(1.2 \mathrm{~b})$, and

(a) the inner limit cycle is stable and the outer one is unstable if there are two limit cycles.

(b) the limit cycle is stable or semi-stable (internally stable and externally unstable) if there is a unique limit cycle.

Proof. Firstly, consider that there are two small limit cycles surrounding $E_{r}$ of system (1.2b). If only one of two limit cycles is semi-stable, by Lemma 3.4 and [21, Theorem 3.4 of Chapter 3.4], at least one stable limit cycle and one unstable limit cycle will be bifurcated from the semi-stable one and the limit cycle of multiple-odd does not disappear as $\mu_{2}$ increases. If both two limit cycles are semi-stable, since they are adjacent to each other, at least four limit cycles will be bifurcated from them. Then one limit cycle is stable and the other one is unstable. By Lemma 2.1, $E_{r}$ is unstable 
when (c4) holds. Thus the inner one is internally stable, indicating the inner limit cycle is stable and the outer one is unstable.

Secondly, if there is a unique small limit cycle surrounding $E_{r}$, we can only obtain it is internally stable. Then, the proof is finished.

Now we are ready to prove the existence of homoclinic loops and give the number of limit cycles in (c4). We first get a homoclinic bifurcation curve in Proposition 3.1. Then a double large limit cycle bifurcation curve and a small large limit bifurcation curve are obtained in Propositions 3.2 and 3.3 respectively.

Proposition 3.1. There is a decreasing $C^{\infty}$ function $\varphi\left(\mu_{1}\right)$ such that $-2\left(12 \mu_{1}^{3}\right)^{1 / 4} / 3<\varphi\left(\mu_{1}\right)<0$ and

(a) system (1.2b) exhibits one figure-eight loop if and only if $\mu_{2}=\varphi\left(\mu_{1}\right)$;

(b) when $\mu_{2}=\varphi\left(\mu_{1}\right)$, system (1.2b) exhibits three limit cycles, where two limit cycles are stable and small, another one is stable and large;

(c) when $\mu_{2}=\varphi\left(\mu_{1}\right)-\epsilon$, system (1.2b) exhibits five limit cycles, where four limit cycles are small (two of them surround $E_{r}$, the inner limit cycle is stable and the outer one is unstable), another one is stable and large;

(d) when $\mu_{2}=\varphi\left(\mu_{1}\right)+\epsilon$, system (1.2b) has four limit cycles, where two limit cycles are stable and small, another two are large (the inner limit cycle is unstable and the outer one is stable),

where $\epsilon>0$ is sufficiently small.

Proof. We first prove the statements (a)-(d). As shown above Lemma $3.4, W_{\mu_{1}, \mu_{2}}^{+}$and $W_{\mu_{1}, \mu_{2}}^{-}$ are the unstable manifold and the stable manifold on the right-hand side of system $(1.2 \mathrm{~b})$ at $E_{0}$. Assume that $W_{\mu_{1}, \mu_{2}}^{+}$and $W_{\mu_{1}, \mu_{2}}^{-}$intersect the $x$-axis at $x_{A}^{0}\left(\mu_{1}, \mu_{2}\right), x_{B}^{0}\left(\mu_{1}, \mu_{2}\right)$ respectively. By the symmetry, the existence of one figure-eight loop for system $(1.2 \mathrm{~b})$ means $x_{A}^{0}\left(\mu_{1}, \mu_{2}\right)-x_{B}^{0}\left(\mu_{1}, \mu_{2}\right)=0$.

When $\mu_{2}=0, E_{r}$ is a stable weak center by Lemma 2.1. It follows from $\left.\dot{y}\right|_{y=0}=\mu_{1} x-x^{3}<0$ for $x>\sqrt{\mu_{1}}$ that $x_{A}^{0}\left(\mu_{1}, 0\right)-x_{B}^{0}\left(\mu_{1}, 0\right)<0$. If not, system $(1.2 \mathrm{~b})$ exhibits a homoclinic loop or at least one small limit cycle by Poincaré-Bendixson Theorem, which conflicts with Lemma 3.1. Similarly, when $\mu_{2}=-2\left(12 \mu_{1}^{3}\right)^{1 / 4} / 3, E_{r}$ is unstable by Lemma 2.1 and system (1.2b) exhibits neither homoclinic loops or limit cycles by Lemma 3.5 , yielding $x_{A}^{0}\left(\mu_{1},-2\left(12 \mu_{1}^{3}\right)^{1 / 4} / 3\right)-x_{B}^{0}\left(\mu_{1},-2\left(12 \mu_{1}^{3}\right)^{1 / 4} / 3\right)>$ 0 .

By Lemma 3.4, there exists a unique function $\mu_{2}=\varphi\left(\mu_{1}\right) \in\left(0,-2\left(12 \mu_{1}^{3}\right)^{1 / 4} / 3\right)$ such that $x_{A}^{0}\left(\mu_{1}, \varphi\left(\mu_{1}\right)\right)-x_{B}^{0}\left(\mu_{1}, \varphi\left(\mu_{1}\right)\right)=0$, implying that system (1.2b) has a unique homoclinic loop in the right half plane. The statement (a) is proven.

Notice that $\operatorname{tr} J_{0}=\mu_{2}>0$, where $J_{0}$ is the Jacobian matrix at $E_{0}$ and defined in (2.1). By [7, Theorem 3.3], the homoclinic loop of $E_{0}$ is asymptotically unstable. Then if there are limit cycles 
for system (1.2b), the outermost small limit cycle must be externally stable and the innermost large limit cycle must be internally stable.

By Lemma 2.1, $E_{r}$ is unstable when $\mu_{2}=-2\left(12 \mu_{1}^{3}\right)^{1 / 4} / 3<0$. Combining the instability of the homoclinic loop, there is at least one small limit cycle surrounding $E_{r}$. Moreover, since the small limit cycle which is closest to the homoclinic loop is externally stable, by Lemma 3.12 there is a unique small limit cycle surrounding $E_{r}$, which is stable. By the symmetry, there is a unique small limit cycle surrounding $E_{l}$, which is stable.

By Lemma 2.3, all orbits of system (1.2b) are positively bounded. Combining the instability of the homoclinic loop, we get the existence of large limit cycles of system (1.2b). Since the large limit cycle closest to the homoclinic loop is internally stable, by Lemma 3.8 there is a unique large limit cycle of system $(1.2 \mathrm{~b})$, which is stable. The statement (b) is proven.

Since the homoclinic loop of $E_{0}$ is asymptotically unstable when $\mu_{2}=\varphi\left(\mu_{1}\right)$, there exists a $d \in\left(0, \sqrt{\mu_{1}}\right)$ such that $x_{\tilde{A}}^{d}\left(\mu_{1}, \varphi\left(\mu_{1}\right)\right)-x_{\tilde{B}}^{d}\left(\mu_{1}, \varphi\left(\mu_{1}\right)\right)<0$. By continuous dependence of the solution on parameters,

$$
x_{\tilde{A}}^{d}\left(\mu_{1}, \varphi\left(\mu_{1}\right)-\epsilon\right)-x_{\tilde{B}}^{d}\left(\mu_{1}, \varphi\left(\mu_{1}\right)-\epsilon\right)<0
$$

for sufficiently small $\epsilon>0$. On the one hand, by Lemma 3.4,

$$
x_{\tilde{A}}^{0}\left(\mu_{1}, \varphi\left(\mu_{1}\right)-\epsilon\right)-x_{\tilde{B}}^{0}\left(\mu_{1}, \varphi\left(\mu_{1}\right)-\epsilon\right)>0 .
$$

Then by the continuous dependence of the solution on initial values, there exists a $d_{1} \in(0, d)$ such that

$$
x_{\tilde{A}}^{d_{1}}\left(\mu_{1}, \varphi\left(\mu_{1}\right)-\epsilon\right)-x_{\tilde{B}}^{d_{1}}\left(\mu_{1}, \varphi\left(\mu_{1}\right)-\epsilon\right)=0 .
$$

On the other hand, since $E_{r}$ is unstable when $\mu_{2}=\varphi\left(\mu_{1}\right)-\epsilon<0$, by Poincaré-Bendixson Theorem there exists a $d_{2} \in\left(d, \sqrt{\mu_{1}}\right)$ such that

$$
x_{\tilde{A}}^{d_{2}}\left(\mu_{1}, \varphi\left(\mu_{1}\right)-\epsilon\right)-x_{\tilde{B}}^{d_{2}}\left(\mu_{1}, \varphi\left(\mu_{1}\right)-\epsilon\right)=0 .
$$

That implies at least two small limit cycles exist surround $E_{r}$. By Lemma 3.12, there are exactly two small limit cycles surrounding $E_{r}$, the inner limit cycle is stable and the outer one is unstable. By the symmetry, there are also two small limit cycles surrounding $E_{l}$, the inner limit cycle is stable and the outer one is unstable.

The existence of large limit cycles comes from (3.27) and the positive boundedness of all the orbits. Moreover, (3.27) also implies that the innermost large limit cycle is internally stable. By Lemma 3.8, the large limit cycle is unique and stable. The statement (c) is proven.

By the instability of the homoclinic loop of $E_{0}$ when $\mu_{2}=\varphi\left(\mu_{1}\right)$, there exists a $c>0$ such that $x_{A}^{c}\left(\mu_{1}, \varphi\left(\mu_{1}\right)\right)-x_{B}^{c}\left(\mu_{1}, \varphi\left(\mu_{1}\right)\right)>0$. By continuous dependence of the solution on parameters,

$$
x_{A}^{c}\left(\mu_{1}, \varphi\left(\mu_{1}\right)+\epsilon\right)-x_{B}^{c}\left(\mu_{1}, \varphi\left(\mu_{1}\right)+\epsilon\right)>0
$$

for sufficiently small $\epsilon>0$. On the one hand, by Lemma 3.4,

$$
x_{A}^{0}\left(\mu_{1}, \varphi\left(\mu_{1}\right)+\epsilon\right)-x_{B}^{0}\left(\mu_{1}, \varphi\left(\mu_{1}\right)+\epsilon\right)<0 .
$$


Then by the continuous dependence of the solution on initial values, there exists a $c_{1} \in(0, c)$ such that

$$
x_{A}^{c_{1}}\left(\mu_{1}, \varphi\left(\mu_{1}\right)+\epsilon\right)-x_{B}^{c_{1}}\left(\mu_{1}, \varphi\left(\mu_{1}\right)+\epsilon\right)=0 .
$$

On the other hand, since all the orbits are positively bounded by Lemma 2.3, by Poincaré-Bendixson Theorem there exists a $c_{2} \in(c, \infty)$ such that

$$
x_{A}^{c_{2}}\left(\mu_{1}, \varphi\left(\mu_{1}\right)+\epsilon\right)-x_{B}^{c_{2}}\left(\mu_{1}, \varphi\left(\mu_{1}\right)+\epsilon\right)=0 .
$$

That implies at least two large limit cycles exist for system (1.2b). By Lemma 3.8, there are exactly two large limit cycles, the inner limit cycle is unstable and the outer one is stable.

The existence of small limit cycles surrounding $E_{r}$ comes from (3.28) and the instability of $E_{r}$. Moreover, (3.28) also implies that the outermost small limit cycle surrounding $E_{r}$ is externally stable. By Lemma 3.12 the small limit cycle surrounding $E_{r}$ is unique and stable. By symmetry, there is also a unique small limit cycle surrounding $E_{l}$, which is stable. The statement (d) is proven.

In what follows, we investigate the smoothness of $\varphi\left(\mu_{1}\right)$. Consider system (3.18), which has the same topological structure as system (1.2b). From the statement (a), system (3.18) exhibits a figure-eight homoclinic loop connecting $(-1,0)$ and $(1,0)$ when $\mu_{2}=\varphi\left(\mu_{1}\right)$. As $\mu_{1}$ changes to $\mu_{1}+\delta$, to keep the existence of the figure-eight homoclinic loop, there exists a $\epsilon=\varphi\left(\mu_{1}+\delta\right)-\varphi\left(\mu_{1}\right)$ such that $\chi_{A}^{0}\left(\mu_{1}+\delta, \mu_{2}+\epsilon\right)-\chi_{B}^{0}\left(\mu_{1}+\delta, \mu_{2}+\epsilon\right)=0$. From (3.21) and (3.22), one can calculate that when $\delta>0$

$$
\begin{aligned}
\mathcal{T}_{1} & =\chi_{A}^{0}\left(\mu_{1}+\delta, \mu_{2}\right)-\chi_{A}^{0}\left(\mu_{1}, \mu_{2}\right)=\int_{\chi_{A}^{0}\left(\mu_{1}, \mu_{2}\right)}^{\chi_{A}^{0}\left(\mu_{1}+\delta, \mu_{2}\right)} d x \\
& =\int_{0}^{y_{\mu_{1}, \mu_{2}}^{+}\left(\chi_{A}^{0}\left(\mu_{1}+\delta, \mu_{2}\right)\right)-y_{\mu_{1}+\delta, \mu_{2}}^{+}\left(\chi_{A}^{0}\left(\mu_{1}+\delta, \mu_{2}\right)\right)} \frac{y}{x-x^{3}+\mu_{2} y / \sqrt{\mu_{1}}-\sqrt{\mu_{1}} y^{2}} d y \\
& =\left[\frac{y^{2}}{2\left(\chi_{A}^{0}\left(\mu_{1}, \mu_{2}\right)-\left(\chi_{A}^{0}\left(\mu_{1}, \mu_{2}\right)\right)^{3}\right)}+O\left(y^{3}\right)\right]_{0}^{y_{\mu_{1}, \mu_{2}}^{+}\left(\chi_{A}^{0}\left(\mu_{1}+\delta, \mu_{2}\right)\right)-y_{\mu_{1}+\delta, \mu_{2}}^{+}\left(\chi_{A}^{0}\left(\mu_{1}+\delta, \mu_{2}\right)\right)} \\
& =\frac{\delta^{2}\left(\int_{0}^{\chi_{A}^{0}\left(\mu_{1}+\delta, \mu_{2}\right)}\left(\frac{\mu_{2}}{\sqrt{\mu_{1}\left(\mu_{1}+\delta\right)}}-y_{\mu_{1}+\delta, \mu_{2}}^{+}(\tau)\right) \exp \left\{\int_{\tau}^{\chi_{A}^{0}\left(\mu_{1}+\delta, \mu_{2}\right)} K_{3}(\eta) d \eta\right\} d \tau\right)^{2}}{2\left(\sqrt{\mu_{1}+\delta}+\sqrt{\mu_{1}}\right)^{2}\left(\chi_{A}^{0}\left(\mu_{1}, \mu_{2}\right)-\left(\chi_{A}^{0}\left(\mu_{1}, \mu_{2}\right)\right)^{3}\right)}+O\left(\delta^{3}\right)
\end{aligned}
$$

and

$$
\begin{aligned}
\mathcal{T}_{2} & =\chi_{B}^{0}\left(\mu_{1}+\delta, \mu_{2}\right)-\chi_{B}^{0}\left(\mu_{1}, \mu_{2}\right)=\int_{\chi_{B}^{0}\left(\mu_{1}, \mu_{2}\right)}^{\chi_{B}^{0}\left(\mu_{1}+\delta, \mu_{2}\right)} d x \\
& =\int_{y_{\mu_{1}+\delta, \mu_{2}}^{-}\left(\chi_{B}^{0}\left(\mu_{1}, \mu_{2}\right)\right)-y_{\mu_{1}, \mu_{2}}\left(\chi_{B}^{0}\left(\mu_{1}, \mu_{2}\right)\right)}^{0} \frac{y}{x-x^{3}+\mu_{2} y / \sqrt{\mu_{1}}-\sqrt{\mu_{1} y^{2}}} d y \\
& =\left[\frac{y^{2}}{2\left(\chi_{B}^{0}\left(\mu_{1}+\delta, \mu_{2}\right)-\left(\chi_{B}^{0}\left(\mu_{1}+\delta, \mu_{2}\right)\right)^{3}\right)}+O\left(y^{3}\right)\right]_{y_{\mu_{1}+\delta, \mu_{2}}^{-}\left(\chi_{B}^{0}\left(\mu_{1}, \mu_{2}\right)\right)-y_{\mu_{1}, \mu_{2}}^{-}\left(\chi_{B}^{0}\left(\mu_{1}, \mu_{2}\right)\right)}^{0} \\
& =\frac{-\delta^{2}\left(\int_{0}^{\chi_{B}^{0}\left(\mu_{1}, \mu_{2}\right)}\left(\frac{\mu_{2}}{\sqrt{\mu_{1}\left(\mu_{1}+\delta\right)}}+y_{\mu_{1}+\delta, \mu_{2}}^{-}(\tau)\right) \exp \left\{\int_{\tau}^{\chi_{B}^{0}\left(\mu_{1}, \mu_{2}\right)} K_{4}(\eta) d \eta\right\} d \tau,\right)^{2}}{2\left(\sqrt{\mu_{1}+\delta}+\sqrt{\mu_{1}}\right)^{2}\left(\chi_{B}^{0}\left(\mu_{1}+\delta, \mu_{2}\right)-\left(\chi_{B}^{0}\left(\mu_{1}+\delta, \mu_{2}\right)\right)^{3}\right)}+O\left(\delta^{3}\right) .
\end{aligned}
$$

It follows from (3.29) and (3.30) that $\chi_{A}^{0}\left(\mu_{1}+\delta, \mu_{2}\right)-\chi_{B}^{0}\left(\mu_{1}+\delta, \mu_{2}\right)=\mathcal{T}_{1}-\mathcal{T}_{2}<0$. One can check that $\chi_{A}^{0}\left(\mu_{1}+\delta, \mu_{2}+\epsilon\right)<\chi_{A}^{0}\left(\mu_{1}+\delta, \mu_{2}\right)$ and $\chi_{B}^{0}\left(\mu_{1}+\delta, \mu_{2}+\epsilon\right)<\chi_{B}^{0}\left(\mu_{1}+\delta, \mu_{2}\right)$ if $\epsilon>0$ from (3.19) and (3.20). Then $\chi_{A}^{0}\left(\mu_{1}+\delta, \mu_{2}+\epsilon\right)-\chi_{B}^{0}\left(\mu_{1}+\delta, \mu_{2}+\epsilon\right)=0$ implies $\epsilon<0$. Thus,

$$
\mathcal{T}_{3}=\chi_{A}^{0}\left(\mu_{1}+\delta, \mu_{2}+\epsilon\right)-\chi_{A}^{0}\left(\mu_{1}+\delta, \mu_{2}\right)
$$




$$
=\frac{\epsilon^{2}\left(\int_{0}^{\chi_{A}^{0}\left(\mu_{1}+\delta, \mu_{2}\right)} \exp \left\{\int_{\tau}^{\chi_{A}^{0}\left(\mu_{1}+\delta, \mu_{2}\right)} K_{1}^{*}(\eta) d \eta\right\} d \tau\right)^{2}}{2\left(\mu_{1}+\delta\right)\left(\chi_{A}^{0}\left(\mu_{1}+\delta, \mu_{2}+\epsilon\right)-\left(\chi_{A}^{0}\left(\mu_{1}+\delta, \mu_{2}+\epsilon\right)\right)^{3}\right)}+O\left(\epsilon^{3}\right)
$$

and

$$
\begin{aligned}
\mathcal{T}_{4} & =\chi_{B}^{0}\left(\mu_{1}+\delta, \mu_{2}+\epsilon\right)-\chi_{B}^{0}\left(\mu_{1}+\delta, \mu_{2}\right) \\
& =-\frac{\epsilon^{2}\left(\int_{0}^{\chi_{B}^{0}\left(\mu_{1}+\delta, \mu_{2}+\epsilon\right)} \exp \left\{\int_{\tau}^{\chi_{B}^{0}\left(\mu_{1}+\delta, \mu_{2}+\epsilon\right)} K_{2}^{*}(\eta) d \eta\right\} d \tau\right)^{2}}{2\left(\mu_{1}+\delta\right)\left(\chi_{B}^{0}\left(\mu_{1}+\delta, \mu_{2}\right)-\left(\chi_{B}^{0}\left(\mu_{1}+\delta, \mu_{2}\right)\right)^{3}\right)}+O\left(\epsilon^{3}\right)
\end{aligned}
$$

where

$$
K_{1}^{*}(x)=\frac{-x+x^{3}}{y_{\mu_{1}+\delta, \mu_{2}+\epsilon}^{+}(x) y_{\mu_{1}+\delta, \mu_{2}}^{+}(x)}-\sqrt{\mu_{1}+\delta}, \quad K_{2}^{*}(x)=\frac{-x+x^{3}}{y_{\mu_{1}+\delta, \mu_{2}+\epsilon}^{-}(x) y_{\mu_{1}+\delta, \mu_{2}}^{-}(x)}+\sqrt{\mu_{1}+\delta} .
$$

Substituting (3.29)-(3.32) into $\mathcal{T}_{1}+\mathcal{T}_{3}=\mathcal{T}_{2}+\mathcal{T}_{4}$ and taking limits for the both sides as $\delta \rightarrow 0$, it is easy to obtain that $\lim _{\delta \rightarrow 0} \epsilon=0$. Moreover, since $\epsilon<0$ when $\delta>0$, we get

$$
\begin{aligned}
& \varphi^{\prime}\left(\mu_{1}\right)=\lim _{\delta \rightarrow 0^{+}} \frac{\epsilon}{\delta}=\lim _{\delta \rightarrow 0^{+}}-\sqrt{\frac{\left(\mathcal{T}_{1}-\mathcal{T}_{2}\right) / \delta^{2}}{\left(\mathcal{T}_{4}-\mathcal{T}_{3}\right) / \epsilon^{2}}} \\
& =-\sqrt{\frac{\frac{\left(\int_{0}^{\chi_{A}^{0}}\left(\frac{\mu_{2}}{\mu_{1}}-y_{\mu_{1}, \mu_{2}}^{+}(\tau)\right) \exp \left\{\int_{\tau}^{\chi_{A}^{0}} K_{1}^{* *}(\eta) d \eta\right\} d\right)^{2}}{8 \mu_{1}\left(\chi_{A}^{0}-\left(\chi_{A}^{0}\right)^{3}\right)}+\frac{\left(\int_{0}^{\chi_{B}^{0}}\left(\frac{\mu_{2}}{\mu_{1}}+y_{\mu_{1}}^{-}, \mu_{2}(\tau)\right) \exp \left\{\int_{\tau}^{\chi_{B}^{0}} K_{2}^{* *}(\eta) d \eta d \tau,\right)^{2}\right.}{8 \mu_{1}\left(\chi_{B}^{0}-\left(\chi_{B}^{0}\right)^{3}\right)}}{\frac{\left(\int_{0}^{\chi_{A}^{0}} \exp \left\{\int_{\tau}^{\chi_{A}^{0}} K_{1}^{* *}(\eta) d \eta\right\} d \tau\right)^{2}}{2 \mu_{1}\left(\chi_{A}^{0}-\left(\chi_{A}^{0}\right)^{3}\right)}+\frac{\left(\int_{0}^{\chi_{B}^{0}} \exp \left\{\int_{\tau}^{\chi_{B}^{0}} K_{2}^{* *}(\eta) d \eta\right\} d \tau\right)^{2}}{2 \mu_{1}\left(\chi_{B}^{0}-\left(\chi_{B}^{0}\right)^{3}\right)}}}
\end{aligned}
$$

where $\chi_{A}^{0}=\chi_{A}^{0}\left(\mu_{1}, \mu_{2}\right), \chi_{B}^{0}=\chi_{B}^{0}\left(\mu_{1}, \mu_{2}\right), K_{1}^{* *}(x)=\left(-x+x^{3}\right) /\left(y_{\mu_{1}, \mu_{2}}^{+}(x)\right)^{2}-\sqrt{\mu_{1}}$ and $K_{2}^{* *}(x)=$ $\left(-x+x^{3}\right) /\left(y_{\mu_{1}+\delta, \mu_{2}}^{-}(x)\right)^{2}+\sqrt{\mu_{1}}$. When $\delta<0$, we can similarly prove that $\epsilon>0$ and (3.33) still holds. It follows from $(3.33)$ that $\varphi^{\prime}\left(\mu_{1}\right)<0$. The expression $(3.33)$ shows that $\varphi\left(\mu_{1}\right)$ is $C^{1}$. Furthermore, $\varphi^{\prime}\left(\mu_{1}\right)$ has the same smoothness as $\varphi\left(\mu_{1}\right)$, implying the $C^{\infty}$ of $\varphi\left(\mu_{1}\right)$.

Proposition 3.2. There exists a decreasing $C^{0}$ function $\varrho_{1}\left(\mu_{1}\right)$ such that $\varphi\left(\mu_{1}\right)<\varrho_{1}\left(\mu_{1}\right)<0$ and

(a) when $\mu_{2}=\varrho_{1}\left(\mu_{1}\right)$, system (1.2b) exhibits a unique large limit cycle, which is internally unstable, externally stable;

(b) when $\varphi\left(\mu_{1}\right)<\mu_{2}<\varrho_{1}\left(\mu_{1}\right)$, system (1.2b) exhibits two large limit cycles. The inner one is unstable and the outer one is stable;

(c) when $\varrho_{1}\left(\mu_{1}\right)<\mu_{2}<0$, system (1.2b) exhibits no large limit cycles;

(d) when $\varphi\left(\mu_{1}\right)<\mu_{2}<0$, system (1.2b) exhibits two small limit cycles, which are stable.

Proof. We prove the statements (a)-(d) one by one. By Proposition 3.1, system (1.2b) exhibits a unique large limit cycle when $\mu_{2}=\varphi\left(\mu_{1}\right)$. Assume that this large limit cycle crosses the $y$-axis at the point $\left(0, c_{1}\right)$, where $c_{1}>0$. Since $x_{A}^{c}\left(\mu_{1}, \mu_{2}\right)$ and $x_{B}^{c}\left(\mu_{1}, \mu_{2}\right)$ both depend continuously on $c$, the maximum value of $x_{A}^{c}\left(\mu_{1}, \mu_{2}\right)-x_{B}^{c}\left(\mu_{1}, \mu_{2}\right)$ over the interval $\left[0, c_{1}\right]$ exits, denoted by $M\left(\mu_{1}, \mu_{2}\right)$.

We claim that $M\left(\mu_{1}, \mu_{2}\right)$ decreases continuously as $\mu_{2}$ increases. In fact, since $x_{A}^{c}\left(\mu_{1}, \mu_{2}\right)$ and $x_{B}^{c}\left(\mu_{1}, \mu_{2}\right)$ depend continuously on $\mu_{2}$ for any $c \in\left[0, c_{1}\right]$, we get that $M\left(\mu_{1}, \mu_{2}\right)$ is continuous with respect to $\mu_{2}$. By Lemma 3.4, for any $\epsilon>0$,

$$
M\left(\mu_{1}, \mu_{2}-\epsilon\right)-M\left(\mu_{1}, \mu_{2}\right)=M\left(\mu_{1}, \mu_{2}-\epsilon\right)-\left(x_{A}^{c_{0}}\left(\mu_{1}, \mu_{2}\right)-x_{B}^{c_{0}}\left(\mu_{1}, \mu_{2}\right)\right)
$$




$$
\begin{aligned}
& >M\left(\mu_{1}, \mu_{2}-\epsilon\right)-\left(x_{A}^{c_{0}}\left(\mu_{1}, \mu_{2}-\epsilon\right)-x_{B}^{c_{0}}\left(\mu_{1}, \mu_{2}-\epsilon\right)\right) \\
& \geq 0
\end{aligned}
$$

where $c_{0}$ is a point such that $x_{A}^{c}\left(\mu_{1}, \mu_{2}\right)-x_{B}^{c}\left(\mu_{1}, \mu_{2}\right)$ takes the maximum value at $c=c_{0}$.

On the one hand, when $\mu_{2}=0$ there are no limit cycles for system (1.2b) by Lemma 3.1 and $E_{r}$ is stable by Lemma 2.1. Then $x_{A}^{c}\left(\mu_{1}, 0\right)-x_{B}^{c}\left(\mu_{1}, 0\right)<0$ for any $c \in\left[0, c_{1}\right]$, implying that $M\left(\mu_{1}, 0\right)<0$. On the other hand, by Proposition 3.1 the large limit cycle crossing the $y$-axis at $\left(0, c_{1}\right)$ is stable when $\mu_{2}=\varphi\left(\mu_{1}\right)$. Then $x_{A}^{c}\left(\mu_{1}, \varphi\left(\mu_{1}\right)\right)-x_{B}^{c}\left(\mu_{1}, \varphi\left(\mu_{1}\right)\right)>0$ for some $c \in\left(0, c_{1}\right)$, which implies $M\left(\mu_{1}, \varphi\left(\mu_{1}\right)\right)>0$.

It follows from the monotonicity of $M\left(\mu_{1}, \mu_{2}\right)$ with respect to $\mu_{2}$ that there exists a unique $\varrho_{1}\left(\mu_{1}\right) \in\left(\varphi\left(\mu_{1}\right), 0\right)$ such that $M\left(\mu_{1}, \varrho_{1}\left(\mu_{1}\right)\right)=0$. Moreover, $x_{A}^{c}\left(\mu_{1}, \varrho_{1}\left(\mu_{1}\right)\right)-x_{B}^{c}\left(\mu_{1}, \varrho_{1}\left(\mu_{1}\right)\right) \leq 0$ for any $c \in\left[0, c_{1}\right]$ and there exists a $c_{*} \in\left(0, c_{1}\right)$ such that

$$
x_{A}^{c_{*}}\left(\mu_{1}, \varrho_{1}\left(\mu_{1}\right)\right)-x_{B}^{c_{*}}\left(\mu_{1}, \varrho_{1}\left(\mu_{1}\right)\right)=0 .
$$

That implies there is a large limit cycle when $\mu_{2}=\varrho_{1}\left(\mu_{1}\right)$, which is internally unstable and externally stable. By Lemma 3.8, the large limit cycle is unique. The statement (a) holds.

When $\varphi\left(\mu_{1}\right)<\mu_{2}<\varrho_{1}\left(\mu_{1}\right)$, from Lemma 3.4

$$
\begin{aligned}
& x_{A}^{0}\left(\mu_{1}, \mu_{2}\right)-x_{B}^{0}\left(\mu_{1}, \mu_{2}\right)<x_{A}^{0}\left(\mu_{1}, \varphi\left(\mu_{1}\right)\right)-x_{B}^{0}\left(\mu_{1}, \varphi\left(\mu_{1}\right)\right)=0, \\
& x_{A}^{c_{*}}\left(\mu_{1}, \mu_{2}\right)-x_{B}^{c_{*}}\left(\mu_{1}, \mu_{2}\right)>x_{A}^{c_{*}}\left(\mu_{1}, \varrho_{1}\left(\mu_{1}\right)\right)-x_{B}^{c_{*}}\left(\mu_{1}, \varrho_{1}\left(\mu_{1}\right)\right)=0, \\
& x_{A}^{c_{1}}\left(\mu_{1}, \mu_{2}\right)-x_{B}^{c_{1}}\left(\mu_{1}, \mu_{2}\right)<x_{A}^{c_{1}}\left(\mu_{1}, \varphi\left(\mu_{1}\right)\right)-x_{B}^{c_{1}}\left(\mu_{1}, \varphi\left(\mu_{1}\right)\right)=0 .
\end{aligned}
$$

Then there exist $c_{2} \in\left(0, c_{*}\right)$ and $c_{3} \in\left(c_{*}, c_{1}\right)$ such that

$$
x_{A}^{c_{2}}\left(\mu_{1}, \mu_{2}\right)-x_{B}^{c_{2}}\left(\mu_{1}, \mu_{2}\right)=0, \quad x_{A}^{c_{3}}\left(\mu_{1}, \mu_{2}\right)-x_{B}^{c_{3}}\left(\mu_{1}, \mu_{2}\right)=0 .
$$

That implies system (1.2b) exhibits at least two large limit cycles when $\varphi\left(\mu_{1}\right)<\mu_{2}<\varrho_{1}\left(\mu_{1}\right)$. By Lemma 3.8, there are exactly two large limit cycles for system $(1.2 \mathrm{~b})$ when $\varphi\left(\mu_{1}\right)<\mu_{2}<\varrho_{1}\left(\mu_{1}\right)$, where the inner one is unstable and the outer one is stable. The statement (b) holds.

From the monotonicity of $M\left(\mu_{1}, \mu_{2}\right)$, we get $M\left(\mu_{1}, \mu_{2}\right)<0$ when $\varrho_{1}\left(\mu_{1}\right)<\mu_{2}<0$. Then

$$
x_{A}^{c}\left(\mu_{1}, \mu_{2}\right)-x_{B}^{c}\left(\mu_{1}, \mu_{2}\right)<0
$$

for all $c \in\left[0, c_{1}\right]$. By Proposition 3.1 the large limit cycle, which crosses the $y$-axis at the point $\left(0, c_{1}\right)$, is stable and unique when $\mu_{2}=\varphi\left(\mu_{1}\right)$, Thus,

$$
x_{A}^{c}\left(\mu_{1}, \varphi\left(\mu_{1}\right)\right)-x_{B}^{c}\left(\mu_{1}, \varphi\left(\mu_{1}\right)\right)<0
$$

for all $c \in\left(c_{1}, \infty\right)$. Therefore,

$$
x_{A}^{c}\left(\mu_{1}, \mu_{2}\right)-x_{B}^{c}\left(\mu_{1}, \mu_{2}\right)<x_{A}^{c}\left(\mu_{1}, \varphi\left(\mu_{1}\right)\right)-x_{B}^{c}\left(\mu_{1}, \varphi\left(\mu_{1}\right)\right)<0
$$

for all $c \in\left(c_{1}, \infty\right)$ when $\varphi\left(\mu_{1}\right)<\varrho_{1}\left(\mu_{1}\right)<\mu_{2}<0$. From (3.34) and (3.35), system (1.2b) exhibits no large limit cycles when $\varrho_{1}\left(\mu_{1}\right)<\mu_{2}<0$. The statement (c) holds. 
By Lemma 3.4,

$$
x_{A}^{0}\left(\mu_{1}, \mu_{2}\right)-x_{B}^{0}\left(\mu_{1}, \mu_{2}\right)<x_{A}^{0}\left(\mu_{1}, \varphi\left(\mu_{1}\right)\right)-x_{B}^{0}\left(\mu_{1}, \varphi\left(\mu_{1}\right)\right)=0
$$

when $\varphi\left(\mu_{1}\right)<\mu_{2}<0$. Then similar as in the proof of the statement (d) of Proposition 3.1, the existence of small limit cycles comes from (3.36) and the instability of $E_{r}$. Moreover, (3.36) also implies that the outermost small limit cycle is externally stable. By Lemma 3.12 and the symmetry of system $(1.2 \mathrm{~b})$, there are two limit cycles for system $(1.2 \mathrm{~b})$, which are both stable. The statement (d) holds.

Finally, we show that $\varrho_{1}\left(\mu_{1}\right)$ is decreasing and $C^{0}$. Consider system (3.18), which has the same topological structure as system (1.2b). By Lemma 3.10, $\chi_{A}^{c}\left(\mu_{1}, \mu_{2}\right)-\chi_{B}^{c}\left(\mu_{1}, \mu_{2}\right)$ decreases continuously as $\mu_{1}$ increases. When $\delta>0$, one can check that

$$
0 \geq \chi_{A}^{c}\left(\mu_{1}, \varrho_{1}\left(\mu_{1}\right)\right)-\chi_{B}^{c}\left(\mu_{1}, \varrho_{1}\left(\mu_{1}\right)\right)>\chi_{A}^{c}\left(\mu_{1}+\delta, \varrho_{1}\left(\mu_{1}\right)\right)-\chi_{B}^{c}\left(\mu_{1}+\delta, \varrho_{1}\left(\mu_{1}\right)\right)
$$

for all $c \in[0, \infty)$, implying that there are no large limit cycles for system (3.18) when $\mu_{1}=\mu_{1}+\delta$, $\mu_{2}=\varrho_{1}\left(\mu_{1}\right)$. By the statement (c), we get $\varrho_{1}\left(\mu_{1}\right)>\varrho_{1}\left(\mu_{1}+\delta\right)$ and $\varrho_{1}\left(\mu_{1}\right)$ is decreasing. Moreover, coming back to system $(1.2 \mathrm{~b}), x_{A}^{c}\left(\mu_{1}, \mu_{2}\right)-x_{B}^{c}\left(\mu_{1}, \mu_{2}\right)$ also depends continuously on $\mu_{1}$. Therefore,

$$
\begin{aligned}
\lim _{\delta \rightarrow 0}\left(x_{A}^{c}\left(\mu_{1}, \varrho_{1}\left(\mu_{1}+\delta\right)\right)-x_{B}^{c}\left(\mu_{1}, \varrho_{1}\left(\mu_{1}+\delta\right)\right)\right) & =\lim _{\delta \rightarrow 0}\left(x_{A}^{c}\left(\mu_{1}+\delta, \varrho_{1}\left(\mu_{1}+\delta\right)\right)-x_{B}^{c}\left(\mu_{1}+\delta, \varrho_{1}\left(\mu_{1}+\delta\right)\right)\right) \\
& =0
\end{aligned}
$$

for any $c \in\left[0, c_{1}\right]$, implying that

$$
\lim _{\delta \rightarrow 0} M\left(\mu_{1}, \varrho_{1}\left(\mu_{1}+\delta\right)\right)=0=M\left(\mu_{1}, \varrho_{1}\left(\mu_{1}\right)\right) .
$$

By the uniqueness of $\varrho_{1}\left(\mu_{1}\right)$, we get that $\lim _{\delta \rightarrow 0} \varrho_{1}\left(\mu_{1}+\delta\right)-\varrho_{1}\left(\mu_{1}\right)=0$, which completes the proof.

Proposition 3.3. There exists a decreasing $C^{0}$ function $\varrho_{2}\left(\mu_{1}\right)$ such that $-2\left(12 \mu_{1}^{3}\right)^{1 / 4} / 3<\varrho_{2}\left(\mu_{1}\right)<$ $\varphi\left(\mu_{1}\right)$ and

(a) when $\mu_{2}=\varrho_{2}\left(\mu_{1}\right)$, system (1.2b) exhibits two small limit cycles. One of them surrounds $E_{r}$, which is internally stable, externally unstable;

(b) when $\varrho_{2}\left(\mu_{1}\right)<\mu_{2}<\varphi\left(\mu_{1}\right)$, system (1.2b) exhibits four small limit cycles. Two of them surround $E_{r}$, where the inner one is stable and the outer one is unstable;

(c) when $-2\left(12 \mu_{1}^{3}\right)^{1 / 4} / 3<\mu_{2}<\varrho_{2}\left(\mu_{1}\right)$, system (1.2b) exhibits no small limit cycles;

(d) when $-2\left(12 \mu_{1}^{3}\right)^{1 / 4} / 3<\mu_{2}<\varphi\left(\mu_{1}\right)$, system (1.2b) exhibits a unique large limit cycle, which is stable.

Proof. We first prove the statements (a)-(d). For the statement (a), by Proposition 3.1 there is a unique small limit cycle surrounding $E_{r}$ when $\mu_{2}=\varphi\left(\mu_{1}\right)$. Assume that this small limit cycle crosses the $x$-axis at the point $\left(d_{1}, 0\right)$, where $0<d_{1}<\sqrt{\mu_{1}}$. Consider the minimum value of $x_{\tilde{A}}^{d}\left(\mu_{1}, \mu_{2}\right)-x_{\tilde{B}}^{d}\left(\mu_{1}, \mu_{2}\right)$ over the interval $\left[0, d_{1}\right]$. Since $x_{\tilde{A}}^{d}\left(\mu_{1}, \mu_{2}\right)$ and $x_{\tilde{B}}^{d}\left(\mu_{1}, \mu_{2}\right)$ both depend 
continuously on $d$, the minimum value of $x_{\tilde{A}}^{d}\left(\mu_{1}, \mu_{2}\right)-x_{\tilde{B}}^{d}\left(\mu_{1}, \mu_{2}\right)$ over the interval $\left[0, d_{1}\right]$ exits, denoted by $m\left(\mu_{1}, \mu_{2}\right)$.

We claim that $m\left(\mu_{1}, \mu_{2}\right)$ decreases continuously as $\mu_{2}$ increases. In fact, it is easy to check that $m\left(\mu_{1}, \mu_{2}\right)$ is continuous with respect to $\mu_{2}$ because both $x_{\tilde{A}}^{d}\left(\mu_{1}, \mu_{2}\right)$ and $x_{\tilde{B}}^{d}\left(\mu_{1}, \mu_{2}\right)$ depend continuously on $\mu_{2}$ for any $d \in\left[0, d_{1}\right]$. By Lemma 3.4, for any $\epsilon>0$,

$$
\begin{aligned}
m\left(\mu_{1}, \mu_{2}+\epsilon\right)-m\left(\mu_{1}, \mu_{2}\right) & =m\left(\mu_{1}, \mu_{2}+\epsilon\right)-\left(x_{\tilde{A}}^{d_{0}}\left(\mu_{1}, \mu_{2}\right)-x_{\tilde{B}}^{d_{0}}\left(\mu_{1}, \mu_{2}\right)\right) \\
& <m\left(\mu_{1}, \mu_{2}+\epsilon\right)-\left(x_{\tilde{A}}^{d_{0}}\left(\mu_{1}, \mu_{2}+\epsilon\right)-x_{\tilde{B}}^{d_{0}}\left(\mu_{1}, \mu_{2}+\epsilon\right)\right) \\
& \leq 0
\end{aligned}
$$

where $d_{0}$ is the point such that $x_{\tilde{A}}^{d}\left(\mu_{1}, \mu_{2}\right)-x_{\tilde{B}}^{d}\left(\mu_{1}, \mu_{2}\right)$ takes the minimum value at $d=d_{0}$.

On the one hand, $x_{\tilde{A}}^{d}\left(\mu_{1},-2\left(12 \mu_{1}^{3}\right)^{1 / 4} / 3\right)-x_{\tilde{B}}^{d}\left(\mu_{1},-2\left(12 \mu_{1}^{3}\right)^{1 / 4} / 3\right)>0$ for any $d \in\left[0, d_{1}\right]$, as shown in (3.8). Thus, $m\left(\mu_{1},-2\left(12 \mu_{1}^{3}\right)^{1 / 4} / 3\right)>0$. On the other hand, by Proposition 3.1 the small limit cycle crossing the $x$-axis at the point $\left(d_{1}, 0\right)$ is stable when $\mu_{2}=\varphi\left(\mu_{1}\right)$. Then $x_{\tilde{A}}^{d}\left(\mu_{1}, \varphi\left(\mu_{1}\right)\right)-x_{\tilde{B}}^{d}\left(\mu_{1}, \varphi\left(\mu_{1}\right)\right)<0$ for some $d \in\left(0, d_{1}\right)$. Thus, $m\left(\mu_{1}, \varphi\left(\mu_{1}\right)\right)<0$.

It follows from the monotonicity of $m\left(\mu_{1}, \mu_{2}\right)$ with respect to $\mu_{2}$ that there exists a unique $\varrho_{2}\left(\mu_{1}\right) \in\left(-2\left(12 \mu_{1}^{3}\right)^{1 / 4} / 3, \varphi\left(\mu_{1}\right)\right)$ such that $m\left(\mu_{1}, \varrho_{2}\left(\mu_{1}\right)\right)=0$. Thus, $x_{\tilde{A}}^{d}\left(\mu_{1}, \varrho_{2}\left(\mu_{1}\right)\right)-x_{\tilde{B}}^{d}\left(\mu_{1}, \varrho_{2}\left(\mu_{1}\right)\right) \geq$ 0 for any $d \in\left[0, d_{1}\right]$ and there exists a $d_{*} \in\left(0, d_{1}\right)$ such that

$$
x_{\tilde{A}}^{d_{*}}\left(\mu_{1}, \varrho_{2}\left(\mu_{1}\right)\right)-x_{\tilde{B}}^{d_{*}}\left(\mu_{1}, \varrho_{2}\left(\mu_{1}\right)\right)=0 .
$$

That implies there is a unique limit cycle crossing the interval $0<x<d_{1}$ when $\mu_{2}=\varrho_{2}\left(\mu_{1}\right)$, which is internally stable, externally unstable. By Lemma 3.12, system (1.2b) exhibits a unique limit cycle surrounding $E_{r}$ when $\mu_{2}=\varrho_{2}\left(\mu_{1}\right)$. The statement (a) is proven.

For the statement (b), from Lemma 3.4 it is easy to check that

$$
\begin{gathered}
x_{\tilde{A}}^{0}\left(\mu_{1}, \mu_{2}\right)-x_{\tilde{B}}^{0}\left(\mu_{1}, \mu_{2}\right)>x_{\tilde{A}}^{0}\left(\mu_{1}, \varphi\left(\mu_{1}\right)\right)-x_{\tilde{B}}^{0}\left(\mu_{1}, \varphi\left(\mu_{1}\right)\right)=0, \\
x_{\tilde{A}}^{d_{*}}\left(\mu_{1}, \mu_{2}\right)-x_{\tilde{B}}^{d_{*}}\left(\mu_{1}, \mu_{2}\right)<x_{\tilde{A}}^{d_{*}}\left(\mu_{1}, \varrho_{2}\left(\mu_{1}\right)\right)-x_{\tilde{B}}^{d_{*}}\left(\mu_{1}, \varrho_{2}\left(\mu_{1}\right)\right)=0 . \\
x_{\tilde{A}}^{d_{1}}\left(\mu_{1}, \mu_{2}\right)-x_{\tilde{B}}^{d_{1}}\left(\mu_{1}, \mu_{2}\right)>x_{\tilde{A}}^{d_{1}}\left(\mu_{1}, \varphi\left(\mu_{1}\right)\right)-x_{\tilde{B}}^{d_{1}}\left(\mu_{1}, \varphi\left(\mu_{1}\right)\right)=0,
\end{gathered}
$$

when $\varrho_{2}\left(\mu_{1}\right)<\mu_{2}<\varphi\left(\mu_{1}\right)$. Thus, there exist $d_{2} \in\left(0, d_{*}\right)$ and $d_{3} \in\left(d_{*}, d_{1}\right)$ such that

$$
x_{\tilde{A}}^{d_{2}}\left(\mu_{1}, \mu_{2}\right)-x_{\tilde{B}}^{d_{2}}\left(\mu_{1}, \mu_{2}\right)=0, \quad x_{\tilde{A}}^{d_{3}}\left(\mu_{1}, \mu_{2}\right)-x_{\tilde{B}}^{d_{3}}\left(\mu_{1}, \mu_{2}\right)=0 .
$$

That means there are at least two small limit cycles surrounding $E_{r}$ when $\varrho_{2}\left(\mu_{1}\right)<\mu_{2}<\varphi\left(\mu_{1}\right)$. By Lemma 3.12, system (1.2b) exhibits two small limit cycles surrounding $E_{r}$ when $\varrho_{2}\left(\mu_{1}\right)<\mu_{2}<$ $\varphi\left(\mu_{1}\right)$, where the inner one is stable and the outer one is unstable. The statement (b) is proven.

For the statement $(\mathbf{c})$, when $-2\left(12 \mu_{1}^{3}\right)^{1 / 4} / 3<\mu_{2}<\varrho_{2}\left(\mu_{1}\right)$ we get $m\left(\mu_{1}, \mu_{2}\right)>0$ from the monotonicity of $m\left(\mu_{1}, \mu_{2}\right)$. Then

$$
x_{\tilde{A}}^{d}\left(\mu_{1}, \mu_{2}\right)-x_{\tilde{B}}^{d}\left(\mu_{1}, \mu_{2}\right)>0
$$


for all $d \in\left[0, d_{1}\right]$. By Proposition 3.1, the small limit cycle surrounding $E_{r}$ is stable and unique when $\mu_{2}=\varphi\left(\mu_{1}\right)$, and it crosses the $x$-axis at the point $\left(d_{1}, 0\right)$. Thus,

$$
x_{\tilde{A}}^{d}\left(\mu_{1}, \varphi\left(\mu_{1}\right)\right)-x_{\tilde{B}}^{d}\left(\mu_{1}, \varphi\left(\mu_{1}\right)\right)>0
$$

for all $d \in\left(d_{1}, \sqrt{\mu_{1}}\right)$. Therefore,

$$
x_{\tilde{A}}^{d}\left(\mu_{1}, \mu_{2}\right)-x_{\tilde{B}}^{d}\left(\mu_{1}, \mu_{2}\right)>x_{\tilde{A}}^{d}\left(\mu_{1}, \varphi\left(\mu_{1}\right)\right)-x_{\tilde{B}}^{d}\left(\mu_{1}, \varphi\left(\mu_{1}\right)\right)>0
$$

for all $d \in\left(d_{1}, \sqrt{\mu_{1}}\right)$ when $\mu_{2}<\varrho_{2}\left(\mu_{1}\right)<\varphi\left(\mu_{1}\right)$. From (3.37) and (3.38), there are no limit cycles surrounding $E_{r}$ when $-2\left(12 \mu_{1}^{3}\right)^{1 / 4} / 3<\mu_{2}<\varrho_{2}\left(\mu_{1}\right)$. The statement (c) is proven.

For the statement (d), by Lemma 3.4

$$
x_{\tilde{A}}^{0}\left(\mu_{1}, \mu_{2}\right)-x_{\tilde{B}}^{0}\left(\mu_{1}, \mu_{2}\right)>x_{\tilde{A}}^{0}\left(\mu_{1}, \varphi\left(\mu_{1}\right)\right)-x_{\tilde{B}}^{0}\left(\mu_{1}, \varphi\left(\mu_{1}\right)\right)=0
$$

when $-2\left(12 \mu_{1}^{3}\right)^{1 / 4} / 3<\mu_{2}<\varphi\left(\mu_{1}\right)$. Then similar as in the proof of the statement (c) of Proposition 3.1, the existence of large limit cycles comes from (3.39) and the positive boundedness of all the orbits. Moreover, (3.39) also implies that the innermost large limit cycle is internally stable. By Lemma 3.8, the large limit cycle is unique and stable. The statement (d) is proven.

Finally, we show that $\varrho_{2}\left(\mu_{1}\right)$ is decreasing and $C^{0}$. Consider system (3.18), which has the same topological structure as system (1.2b). By Lemma 3.10, $\chi_{\tilde{A}}^{d}\left(\mu_{1}, \mu_{2}\right)-\chi_{\tilde{B}}^{d}\left(\mu_{1}, \mu_{2}\right)$ decreases as $\mu_{1}$ increases. When $\delta>0$, one can check that

$$
0 \leq \chi_{\tilde{A}}^{d}\left(\mu_{1}, \varrho_{2}\left(\mu_{1}\right)\right)-\chi_{\tilde{B}}^{d}\left(\mu_{1}, \varrho_{2}\left(\mu_{1}\right)\right)<\chi_{\tilde{A}}^{d}\left(\mu_{1}-\delta, \varrho_{2}\left(\mu_{1}\right)\right)-\chi_{\tilde{B}}^{d}\left(\mu_{1}-\delta, \varrho_{2}\left(\mu_{1}\right)\right)
$$

for all $d \in[0,1)$, implying that there are no small limit cycles for system (3.18) when $\mu_{1}=\mu_{1}-\delta$, $\mu_{2}=\varrho_{2}\left(\mu_{1}\right)$. By the statement (c), we get $\varrho_{2}\left(\mu_{1}\right)<\varrho_{2}\left(\mu_{1}-\delta\right)$ and $\varrho_{2}\left(\mu_{1}\right)$ is decreasing. Moreover, coming back to system $(1.2 \mathrm{~b}), x_{\tilde{A}}^{d}\left(\mu_{1}, \mu_{2}\right)-x_{\tilde{B}}^{d}\left(\mu_{1}, \mu_{2}\right)$ also depends continuously on $\mu_{1}$. Therefore,

$$
\begin{aligned}
\lim _{\delta \rightarrow 0}\left(x_{\tilde{A}}^{d}\left(\mu_{1}, \varrho_{2}\left(\mu_{1}+\delta\right)\right)-x_{\tilde{B}}^{d}\left(\mu_{1}, \varrho_{2}\left(\mu_{1}+\delta\right)\right)\right) & =\lim _{\delta \rightarrow 0}\left(x_{\tilde{A}}^{d}\left(\mu_{1}+\delta, \varrho_{2}\left(\mu_{1}+\delta\right)\right)-x_{\tilde{B}}^{d}\left(\mu_{1}+\delta, \varrho_{2}\left(\mu_{1}+\delta\right)\right)\right) \\
& =0
\end{aligned}
$$

for any $d \in\left[0, d_{1}\right]$, implying that

$$
\lim _{\delta \rightarrow 0} m\left(\mu_{1}, \varrho_{2}\left(\mu_{1}+\delta\right)\right)=0=m\left(\mu_{1}, \varrho_{2}\left(\mu_{1}\right)\right) .
$$

By the uniqueness of $\varrho_{2}\left(\mu_{1}\right)$, we get that $\lim _{\delta \rightarrow 0} \varrho_{2}\left(\mu_{1}+\delta\right)-\varrho_{2}\left(\mu_{1}\right)=0$, which completes the proof.

\section{Proofs of Theorems 1.1 and 1.2}

In this section, we prove Theorems 1.1 and 1.2 under the assumption that there are at most two limit cycles surrounding $E_{r}$.

Proof of Theorem 1.1 The statements (a) and (b) can be obtained from Proposition 2.3. The statements (c), (d) and (e) are directly from Propositions 3.1, 3.2 and 3.3 respectively. 
Proof of Theorem 1.2 From Lemma 2.3, system (1.2b) has two infinite equilibria $I_{y}^{+}$and $I_{y}^{-}$. Moreover, there are infinitely many orbits leaving $I_{y}^{+}$in the direction of the negative $x$-axis and infinitely many orbits leaving $I_{y}^{-}$in the direction of the positive $x$-axis as $t \rightarrow \infty$.

When $\left(\mu_{1}, \mu_{2}\right) \in \mathrm{I}$ or $\left(\mu_{1}, \mu_{2}\right) \in P$, by Lemma 2.2 system $(1.2 \mathrm{~b})$ has a unique finite equilibrium $E_{0}$ which is unstable. From Lemma 3.2 there is a unique limit cycle for system $(1.2 \mathrm{~b})$, which is stable. Thus, the limit cycle is the $\omega$-limit set of all the orbits except $E_{0}$.

When $\left(\mu_{1}, \mu_{2}\right) \in \mathrm{II}$ or $\left(\mu_{1}, \mu_{2}, \mu_{3}\right) \in P_{2}$ or $\left(\mu_{1}, \mu_{2}, \mu_{3}\right) \in H_{2}$, by Lemma 2.2 there is a unique finite equilibrium $E_{0}$ for system (1.2b) which is a stable. Due to Lemma 3.1 there are no closed orbits for system $(1.2 \mathrm{~b})$. Thus, $E_{0}$ is the $\omega$-limit set of all the orbits.

When $\left(\mu_{1}, \mu_{2}\right) \in$ III or $\left(\mu_{1}, \mu_{2}, \mu_{3}\right) \in H_{1}$, by Lemma 2.1 system (1.2b) has three finite equilibria $E_{0}, E_{l}$ and $E_{r}$. Moreover, $E_{0}$ is a saddle, $E_{l}$ and $E_{r}$ are stable. It follows from Lemmas 3.1 that there are no small limit cycles for system (1.2b). Thus, except the stable manifolds of $E_{0}$, all the other orbits approach $E_{l}$ or $E_{r}$ as $t \rightarrow \infty$.

When $\left(\mu_{1}, \mu_{2}\right) \in \mathrm{IV}$, by Lemma 2.1 system $(1.2 \mathrm{~b})$ has three finite equilibria $E_{0}, E_{l}$ and $E_{r}$. Moreover, $E_{0}$ is a saddle, $E_{l}$ and $E_{r}$ are unstable. Proposition 3.2 (c) and (d) show that system (1.2b) exhibits two limit cycles, which are small and stable. Thus, except the stable manifolds of $E_{0}$, all the other orbits approach the small limit cycles surrounding $E_{l}$ or $E_{r}$ as $t \rightarrow \infty$.

When $\left(\mu_{1}, \mu_{2}\right) \in D L_{1}, E_{0}$ is a saddle, $E_{l}$ and $E_{r}$ are unstable by Lemma 2.1. From Proposition 3.2 (a) and (d), there are three limit cycles for system $(1.2 \mathrm{~b})$, where two small ones are stable, the large one is internally unstable and externally stable. Thus, the unstable manifolds of $E_{0}$ approach small limit cycles and the stable manifolds of $E_{0}$ leave from the large limit cycle as $t \rightarrow \infty$.

When $\left(\mu_{1}, \mu_{2}\right) \in \mathrm{V}, E_{0}$ is a saddle, $E_{l}$ and $E_{r}$ are unstable by Lemma 2.1. By Proposition 3.2 (b) and (d), there are four limit cycles for system (1.2b), where two small ones are stable, the inner large one is unstable and the outer large one is stable. Thus, the unstable manifolds of $E_{0}$ approach small limit cycles and the stable manifolds of $E_{0}$ leave from the inner large limit cycle as $t \rightarrow \infty$

When $\left(\mu_{1}, \mu_{2}\right) \in H L, E_{0}$ is a saddle, $E_{l}$ and $E_{r}$ are unstable by Lemma 2.1. According to Proposition 3.1 (a) and (b), system (1.2b) exhibits one figure-eight loop and three limit cycles, where two limit cycles are stable and small, another one is stable and large. Moreover, the figureeight loop is unstable as in the proof of Proposition 3.1.

When $\left(\mu_{1}, \mu_{2}\right) \in \mathrm{VI}, E_{0}$ is a saddle, $E_{l}$ and $E_{r}$ are unstable by Lemma 2.1. On the base of Proposition 3.3 (b) and (d), there are five limit cycles for system (1.2b), where the large one is stable, the inner small ones are stable and the outer small ones are unstable. Thus, the unstable manifolds of $E_{0}$ approach the large limit cycle and the stable manifolds of $E_{0}$ leave from the outer small limit cycles as $t \rightarrow \infty$.

When $\left(\mu_{1}, \mu_{2}\right) \in D L_{2}, E_{0}$ is a saddle, $E_{l}$ and $E_{r}$ are unstable by Lemma 2.1. By Proposition 3.3 (a) and (d), there are three limit cycles for system (1.2b), where the large one is stable, two 
small ones are internally stable, externally unstable. Thus, the unstable manifolds of $E_{0}$ approach the large limit cycle and the stable manifolds of $E_{0}$ leave from small limit cycles as $t \rightarrow \infty$.

When $\left(\mu_{1}, \mu_{2}\right) \in$ VII, $E_{0}$ is a saddle, $E_{l}$ and $E_{r}$ are unstable by Lemma 2.1. From Lemmas 3.5, 3.7 and Proposition 3.3 (c) and (d), system (1.2b) exhibits a unique limit cycle, which is large and stable. Thus, the unstable manifolds of $E_{0}$ approach the large limit cycle as $t \rightarrow \infty$.

\section{$5 \quad$ Numerical examples and discussions}

The phase portraits and bifurcations of $(1.2 \mathrm{~b})$ by numerical simulations are shown in this section. It is to note that the qualitative properties of the system $(1.2 \mathrm{~b})$ at infinity can not be reflected in the numerical simulations.

We first consider the case $\mu_{1} \leq 0$. By Lemma 2.2, system $(1.2 \mathrm{~b})$ exhibits a unique equilibrium $E_{0}$.

Example 1. When $\mu_{1}=-1$ and $\mu_{2}=-1, E_{0}$ is an unstable focus. Moreover, system (1.2b) exhibits a stable limit cycle, as shown in Fig. 16 (a). When $\mu_{1}=-1$ and $\mu_{2}=1, E_{0}$ is a stable focus, as shown in Fig. 16 (b).

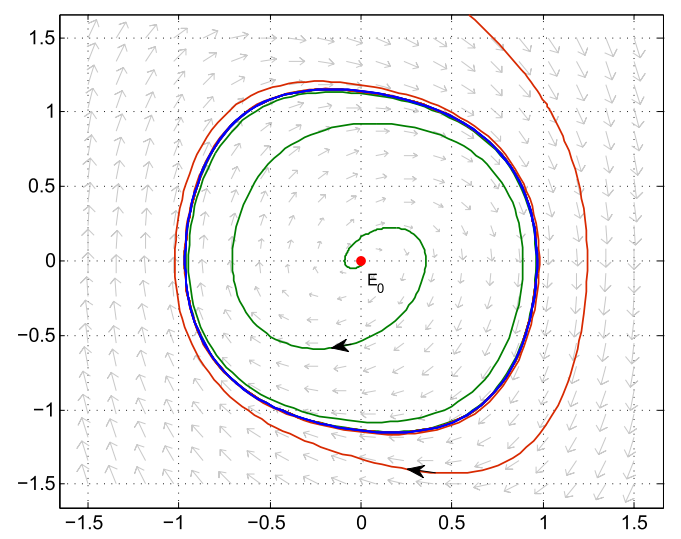

(a) $\mu_{2}=-1$

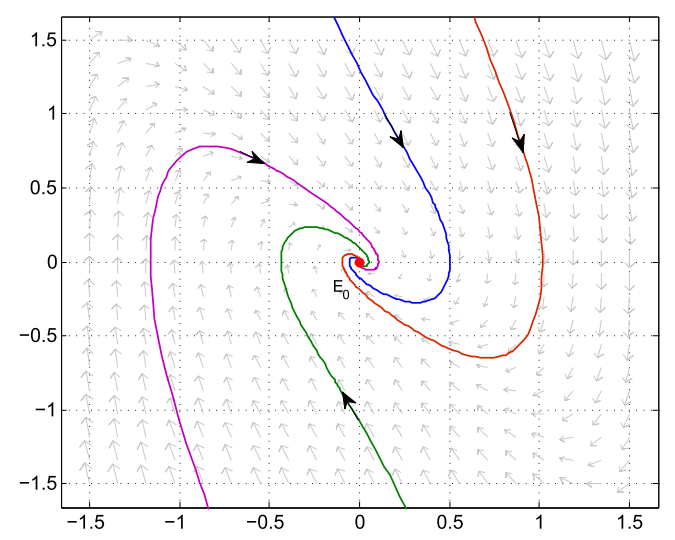

(b) $\mu_{2}=1$

Fig. 16: Numerical phase portraits with one equilibrium when $\mu_{1}=-1$.

Then we consider the case $\mu_{1}>0$. By Lemma 2.1, system (1.2b) exhibits three equilibria $E_{0}$, $E_{l}$ and $E_{r}$. We fix $\mu_{1}=1$ and see how the phase diagram changes as $\mu_{2}$ increases.

Example 2. When $\mu_{1}=1$ and $\mu_{2}=1, E_{0}$ is a saddle, $E_{l}$ and $E_{r}$ are stable foci, as shown in Fig. 17 (a). When $\mu_{1}=1$ and $\mu_{2}=-0.5, E_{0}$ is a saddle, $E_{l}$ and $E_{r}$ are unstable foci. There are two limit cycles for system (1.2b), which are small and stable, as shown in Fig. 17 (b).

We continue to reduce $\mu_{2}$ from -0.5 and find a series of changes in the topological structure of the phase diagram near $\mu_{2}=-0.55$. In the following examples, $E_{0}$ is a saddle, $E_{l}$ and $E_{r}$ are 


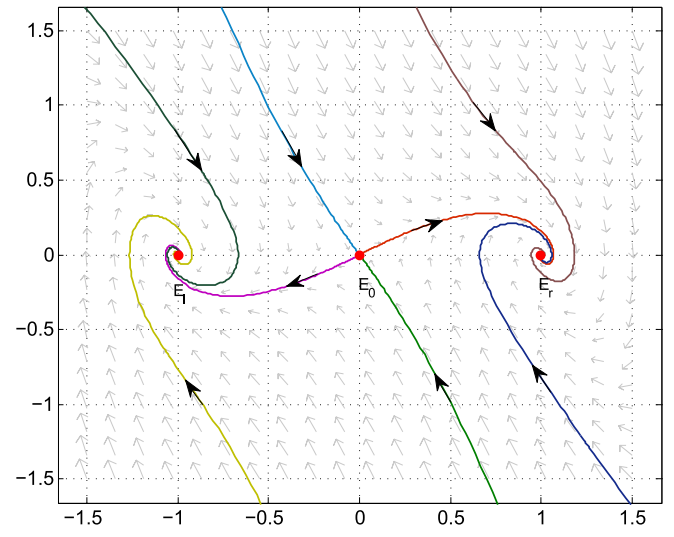

(a) $\mu_{2}=1$

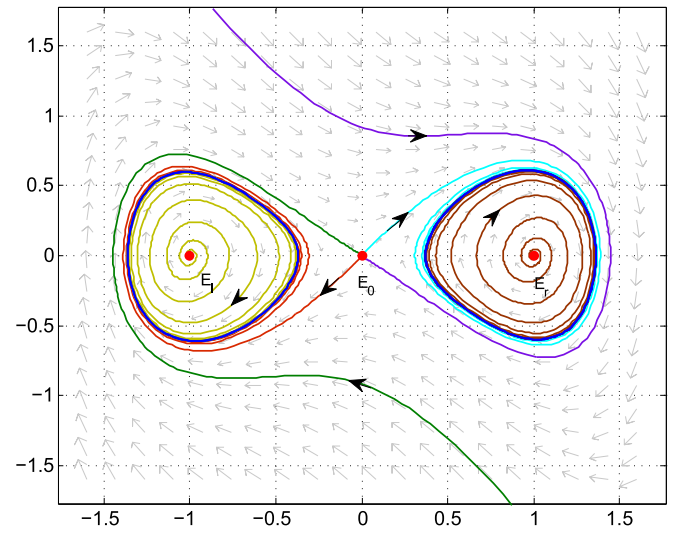

(b) $\mu_{2}=-0.5$

Fig. 17: Numerical phase portraits with three equilibria when $\mu_{1}=1$.

unstable foci. Then we draw stable manifolds and unstable manifolds at $E_{0}$, so as to infer the existence and stability of limit cycles.

Example 3. When $\mu_{1}=1$ and $\mu_{2}=-0.553949$, the $\omega$-limit sets of unstable manifolds at $E_{0}$ are small limit cycles, which are very close to $E_{0}$, as shown in Fig. 18 (a). When $\mu_{1}=1$ and $\mu_{2}=-0.554552$, the $\alpha$-limit set of stable manifolds at $E_{0}$ is a large limit cycle, which is very close to $E_{0}$, as shown in Fig. 18 (b).

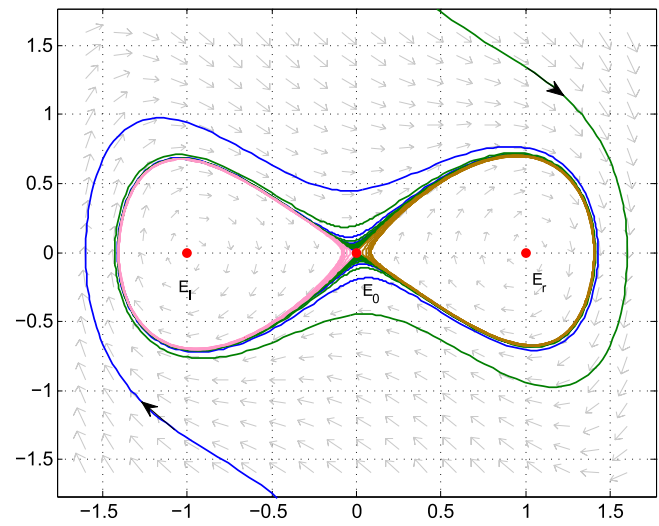

(a) $\mu_{2}=-0.553949$

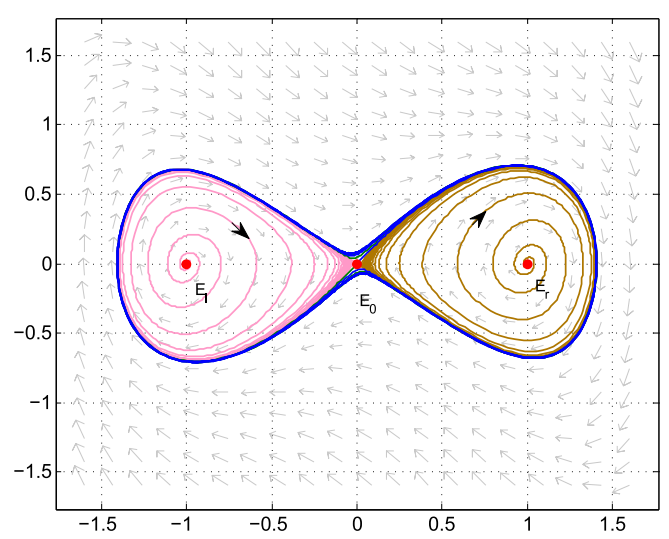

(b) $\mu_{2}=-0.554552$

Fig. 18: Numerical phase portraits with three equilibria when $\mu_{1}=1$.

When $\mu_{1}=1$ and $\mu_{2}$ varies from -0.553949 to -0.554552 , double large limit cycle bifurcations, homoclinic bifurcations and double small limit cycle bifurcations happen one after another.

Example 4. When $\mu_{1}=1$ and $\mu_{2}=-0.554022$, the $\omega$-limit sets of unstable manifolds at $E_{0}$ are small limit cycles and the $\alpha$-limit set of stable manifolds at $E_{0}$ is a large limit cycle, which are all very close to $E_{0}$, as shown in Fig. 19 (a). On the region near $E_{0}$ in Fig. 19 (a), we can see its topological structure, as shown in Fig. 19 (b). When $\mu_{1}=1$ and $\mu_{2}=-0.554205$, system (1.2b) exhibits a figure-eight loop, a large limit cycle and two small limit cycles, as shown in Fig. 


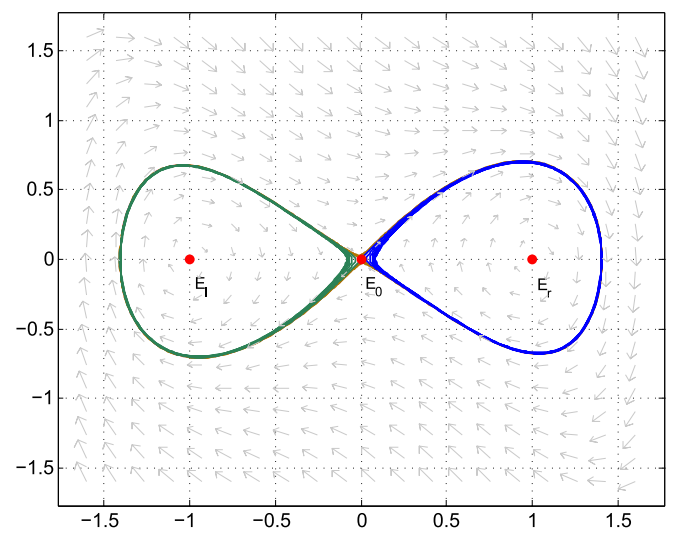

(a) $\mu_{2}=-0.554022$

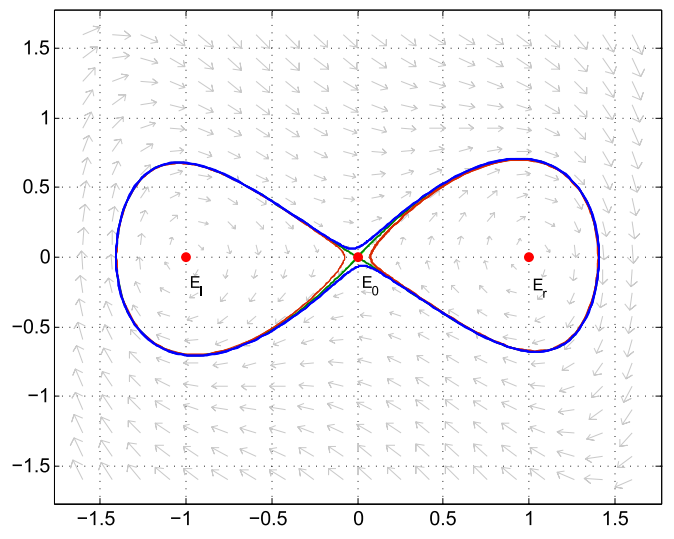

(c) $\mu_{2}=-0.554205$

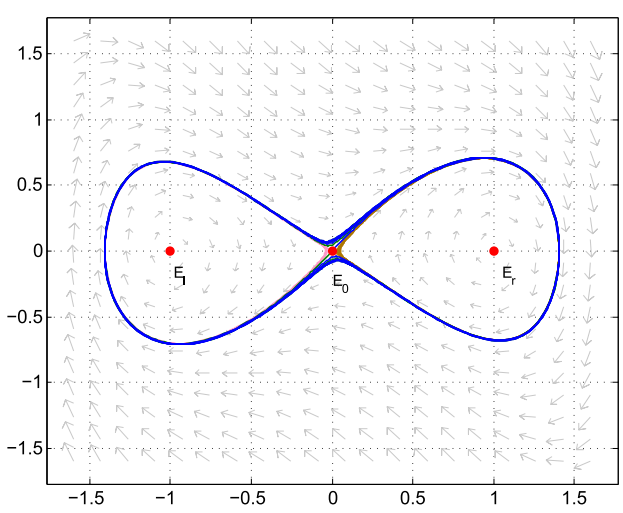

(e) $\mu_{2}=-0.554539$

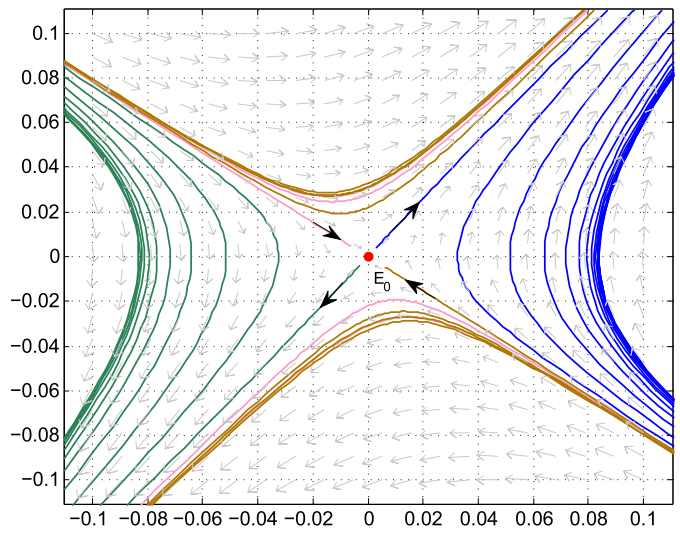

(b) locally enlarged image when $\mu_{2}=-0.554022$

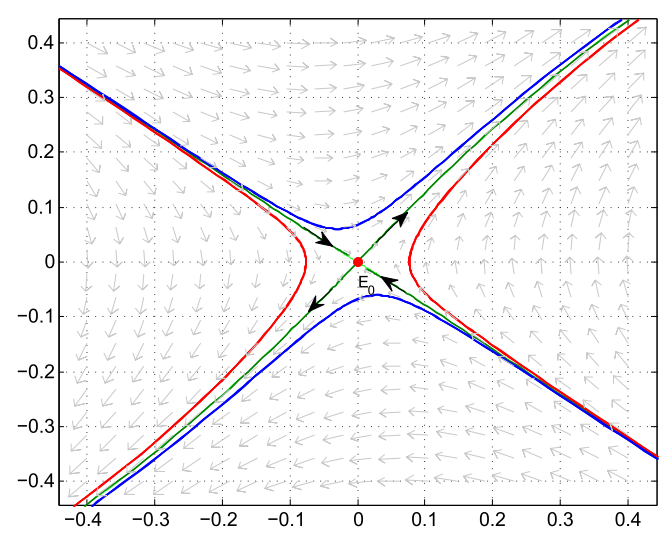

(d) locally enlarged image when $\mu_{2}=-0.554205$

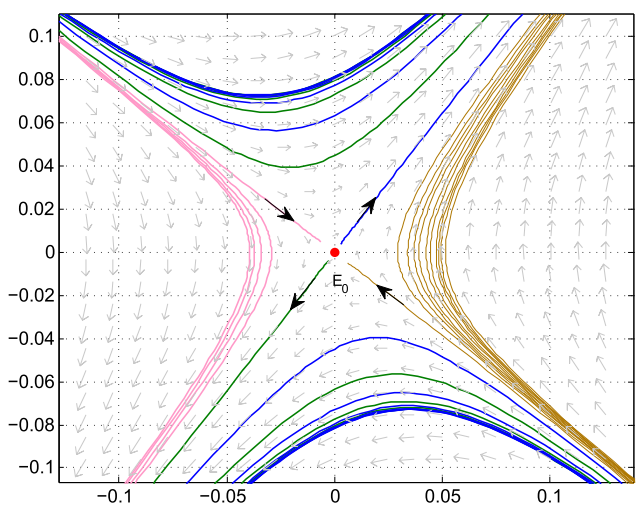

(f) locally enlarged image when $\mu_{2}=-0.554539$

Fig. 19: Numerical phase portraits with three equilibria when $\mu_{1}=1$.

19 (c), and the local topological structure near $E_{0}$ is shown in Fig. 19 (d). When $\mu_{1}=1$ and $\mu_{2}=-0.554539$, the $\omega$-limit sets of unstable manifolds at $E_{0}$ is a large limit cycle and the $\alpha$-limit set of stable manifolds at $E_{0}$ are small limit cycles, which are all very close to $E_{0}$, as shown in Fig. 19 (e). On the region near $E_{0}$ in Fig. 19 (e), we can see its topological structure, as shown in Fig. 19 (f). 
In Fig. 19 (a) and (b), we can get that the large limit cycle is internally unstable and the two small limit cycles (surrounding $E_{l}$ and $E_{r}$ respectively) are external stable when $\mu_{1}=1$ and $\mu_{2}=-0.554022$. Then system (1.2b) exhibits a semi-stable large limit cycle or two large limit cycles which are very close to each other. That implies $\varrho_{1}(1) \approx-0.554022$. In Fig. 19 (c) and (d), we can see a large limit cycle and two small limit cycles coexist with the figure-eight loop and $\varphi(1) \approx-0.554205$. In Fig. 19 (e) and (f), we can get that the large limit cycle is internally stable and the two small limit cycles (surrounding $E_{l}$ and $E_{r}$ respectively) are external unstable when $\mu_{1}=1$ and $\mu_{2}=-0.554539$. Then one semi-stable small limit cycle or two small limit cycles which are very close to each other surround $E_{r}$. That implies $\varrho_{2}(1) \approx-0.554539$. Thus, the bifurcation curves $D L_{1}, H L$ and $D L_{2}$ are very close to each other.

The last example shows that if we continue to reduce $\mu_{2}$, the large limit cycle still exists and is expanding.

Example 5. When $\mu_{1}=1$ and $\mu_{2}=-1, E_{0}$ is a saddle, $E_{l}$ and $E_{r}$ are unstable foci. System (1.2b) exhibits a unique limit cycle, which is large and stable, as shown in Fig. 20.

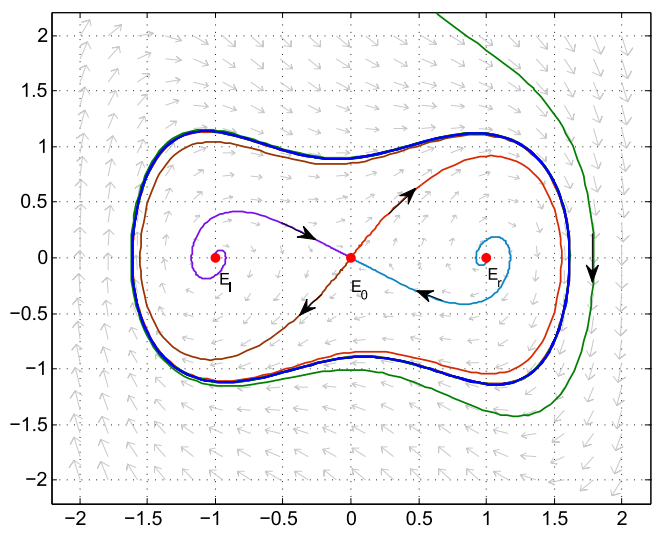

Fig. 20: Numerical phase portraits with three equilibria when $\mu_{1}=1$ and $\mu_{2}=-1$.

\section{Conclusions}

In this section we compare the global dynamics for the focus case of nonsmooth Rayleigh-Duffing oscillator (1.2b) with smooth Rayleigh-Duffing oscillator, smooth van der Pol-Duffing oscillator and nonsmooth van der Pol-Duffing oscillator.

\subsection{Nonsmooth and smooth Rayleigh-Duffing oscillator}

The bifurcation diagram and global phase portraits in the Poincaré disc of a smooth RayleighDuffing oscillator

$$
\dot{x}=y, \quad \dot{y}=-\alpha x-\beta y-x^{3}-y^{3},
$$

where parameters $\alpha$ and $\beta$ are real, are given by [6]. 
The two systems (1.2b) and (6.1) are very similar at finity for both qualitative properties of equilibria and bifurcations of closed orbits. In the case of having unique equilibrium, they both exhibit at most one limit cycle bifurcated from their origins. In the case of having three equilibria, a homoclinic bifurcation, a double small limit cycle bifurcation and a double large limit cycle bifurcation will occur in both systems (1.2b) and (6.1). It is worth mentioning that the gluing bifurcation which happens for (6.1) also appears for nonsmooth Rayleigh-Duffing oscillator (1.2b) from numerical simulations.

However, there are two kinds of differences in the analysis methods of equilibria and closed orbit bifurcations. One is that many classical theories, such as Hopf bifurcation can not be applied directly because the vector field of system $(1.2 \mathrm{~b})$ is only $C^{1}$. In order to overcome these difficulties, some measures like generalized Hopf bifurcation are used for system (1.2b).

The other one is that the upper bound of the number of small limit cycles for system $(1.2 \mathrm{~b})$ can not be obtained so that part of our conclusion is based on an assumption. We did not compare divergence integrals of two closed orbits as in [6] to (6.1), because there is an annular region in the phase diagram such that the monotonicity of divergence integrals is uncertain. However, the complete conclusion for system (6.1) can be given by investigating its Abelian integrals for small parameters and properties of the rotated vector field for general parameters. Since the Abelian integral of system (1.2b) is a combination of an elliptic integral and an elementary function, it is hard to consider the number of zeros. Then there is a theoretical gap in global phase portraits for system (1.2b) though it can not be reflected in the numerical simulation.

The dynamics of systems (1.2b) and (6.1) are different for equilibria at infinity. Due to [6], system (6.1) has four equilibria at infinity. At each equilibrium, there is a parabolic sector surrounding it. For system (1.2b), it has two equilibria at infinity and at each equilibrium there is an elliptical sector and a parabolic sector surrounding it. In conclusion, the bifurcation diagrams of the two oscillators are similar but the analysis of system (1.2b) is indeed more complex than system (6.1), and all global phase portraits in the Poincaré disc are different.

\subsection{Nonsmooth Rayleigh-Duffing oscillator and van der Pol-Duffing oscillator}

The van der Pol-Duffing oscillator

$$
\dot{x}=y-\left(a_{1} x+a_{2} x^{3}\right), \quad \dot{y}=a_{3} x+a_{4} x^{3},
$$

where $a_{2} a_{4} \neq 0$ is investigated in $[8,9]$ for sufficiently small $\left|a_{1}\right|,\left|a_{3}\right|$. When $a_{4}<0$, by $(x, y, t) \rightarrow$ $\left(\sqrt{-a_{4}} x / a_{2},\left(-a_{4}\right)^{3 / 2} y / a_{2}^{2},-a_{2} t / a_{4}\right)$, system $(6.2)$ can be simplified into its focus case

$$
\dot{x}=y-\left(b x+x^{3}\right), \quad \dot{y}=a x-x^{3} .
$$

Global dynamical behaviors of system (6.3) are given in [3] for general $a, b$.

System (1.2b) and (6.3) are both symmetric about the origin. They both have one or three equilibria at finity, depending on different parameters, and pitchfork bifurcations occur. With 
the unique equilibrium, one limit cycle is bifurcated from the origin as the changes of parameters for both system (1.2b) and (6.3). With three equilibria, homoclinic bifurcations appear for some parameters in both system (1.2b) and (6.3). But the number of limit cycles coexisting with the figure eight loop is different for system $(1.2 \mathrm{~b})$ and (6.3). For system (1.2b) there are at least three limit cycles coexisting with the figure eight loop, one is surrounding all the equilibria, the others are surrounding one equilibrium and symmetrically distributed about the origin. For system (6.3) there is one limit cycle coexisting with the figure eight loop, which is surrounding all the equilibria. As a result, with the rupture of figure eight loops, system (1.2b) can generate at least five limit cycles, while system (6.3) can only generate three limit cycles.

Due to [3], system (6.3) has four equilibria at infinity. System (1.2b) has two equilibria at infinity by Lemma 2.3. However, all the orbits are positively bounded for both system (1.2b) and (6.3).

The nonsmooth van der Pol-Duffing oscillator

$$
\dot{x}=y, \quad \dot{y}=\mu_{1} x-x^{3}+\mu_{2} y-\mu_{3}|x| y,
$$

is studied recently by [19] and its bifurcation diagram and global phase portraits are given. The nonsmooth van der Pol-Duffing oscillator and smooth one have similar behaviours at finity. Except that the number of limit cycles are different between system (1.2b) and system (6.4), a bifurcation for equilibria at infinity for system (6.4) cannot occur in system (1.2b).

\section{Appendix}

The saddle case of a Rayleigh-Duffing oscillator

$$
\dot{x}=y, \quad \dot{y}=\alpha x+\beta y+x^{3}-y^{3},
$$

where $\alpha, \beta$ are real, are investigated in [2]. The bifurcation diagram and global phase portraits in the Poincaré disc of system (6.5) are given by [2]. However, in the proof of [2, Lemma 3.4] the set $\left\{(x, y) \in \mathbb{R}: y=y_{1}(x)\right.$ or $\left.y=y_{3}(x), x_{C} \leq x \leq 0\right\}$ was mistakenly regarded as a simple closed curve and the uniqueness and hyperbolicity of closed orbits that can't be verified was presented. In fact, under the condition of [2, Lemma 3.4], the origin is unstable and the 2-saddle loop is internally unstable if it exists. Then system (6.5) exhibits two limit cycles for some parameters though it can not be shown in numerical simulation because of gluing bifurcation.

Here we restate Lemma 3.4 and its proof in [2]. In the following theorem, the number, stability of closed orbits of system (1.2a) are given.

Lemma 6.1. When $0<\beta<-\alpha$, system (6.5) has at most two limit cycles. Moreover,

(a) the inner limit cycle is stable and the outer one is unstable if there are two limit cycles;

(b) the limit cycle is stable or semi-stable (internally stable and externally unstable) if there is a unique limit cycle. 
Proof. Firstly, we prove that there are at most two limit cycles of system (6.5) when $0<\beta<-\alpha$ for sufficiently small $|\alpha|$. By the transformation $(x, y, t) \rightarrow(\sqrt{-\alpha} x,-\alpha y, t / \sqrt{-\alpha})$, system (6.5) can be reduced into

$$
\dot{x}=y, \quad \dot{y}=-x+x^{3}+\frac{\beta}{\sqrt{-\alpha}} y-(-\alpha)^{3 / 2} y^{3},
$$

which has the same topological structure as system (6.5). Obviously, system (6.6) is a perturbation of

$$
\dot{x}=y, \quad \dot{y}=-x+x^{3},
$$

which is a Hamiltonian system with the first integral

$$
H(x, y)=\frac{x^{2}+y^{2}}{2}-\frac{x^{4}}{4} .
$$

The Melnikov function of system (6.6) along $\gamma_{h}: H(x, y)=h$ is

$$
\begin{aligned}
M(h, \alpha, \beta) & =\oint_{\gamma_{h}}\left(\frac{\partial H}{\partial x} \dot{x}+\frac{\partial H}{\partial y} \dot{y}\right) d t=\oint_{\gamma_{h}}\left(\frac{\beta}{\sqrt{-\alpha}} y^{2}-(-\alpha)^{3 / 2} y^{4}\right) d t \\
& =2 \int_{\xi(h)}^{\eta(h)}\left(\frac{\beta}{\sqrt{-\alpha}} y-(-\alpha)^{3 / 2} y^{3}\right) d x=-2(-\alpha)^{3 / 2}\left(\alpha \beta I_{1}+I_{3}\right),
\end{aligned}
$$

where

$$
I_{i}=\int_{\xi(h)}^{\eta(h)} y^{i} d x, \quad i=1,2
$$

$\xi(h)=-\sqrt{1-\sqrt{1-4 h}}$ and $\eta(h)=\sqrt{1-\sqrt{1-4 h}}$. Notice that

$$
I_{3}=\int_{\xi(h)}^{\eta(h)} y\left(2 h-x^{2}+\frac{x^{4}}{2}\right) d x=2 h I_{1}-J_{1}+\frac{J_{2}}{2},
$$

where

$$
J_{i}=\int_{\xi(h)}^{\eta(h)} x^{2 i} y d x, \quad i=0,1,2 .
$$

Clearly, $I_{1}=J_{0}$. Using integrations by parts, we can get another expression of $I_{3}$

$$
I_{3}=\int_{\xi(h)}^{\eta(h)} y^{3} d x=\left.x y^{3}\right|_{\xi(h)} ^{\eta(h)}-\int_{\xi(h)}^{\eta(h)} 3 x y^{2} d y=3 \int_{\xi(h)}^{\eta(h)} x y\left(x-x^{3}\right) d x=3 J_{1}-3 J_{2} .
$$

Eliminating $J_{2}$ from (6.7) and (6.8), we get $I_{3}=12 h I_{1} / 7-3 J_{1} / 7$, yielding that

$$
\frac{I_{3}}{I_{1}}=\frac{12}{7} h-\frac{3 J_{1}}{7 J_{0}} .
$$

Let $P(h):=J_{1} / J_{0}$. Due to [7, Section 4.2], $P(h)$ satisfies Riccati equation

$$
4 h(4 h-1) P^{\prime}(h)=-5 P^{2}+8 h P+4 P-4 h .
$$

Similar to [7, Lemma 2.7 of Chapter 4], one can check that

$$
\lim _{h \rightarrow 0} P^{\prime}(h)=\frac{1}{2}, \quad \lim _{h \rightarrow 1 / 4} P^{\prime}(h)=\infty, \quad P^{\prime}(h)>0 \quad \text { when } 0<h<\frac{1}{4} .
$$


Moreover, we claim that $P^{\prime \prime}(h)>0$ when $0<h<1 / 4$. In fact, differentiating the both sides of equation (6.10), we get

$$
\begin{aligned}
& 2 h(4 h-1) P^{\prime \prime}=(-5 P-12 h+4) P^{\prime}+4 P-2, \\
& 2 h(4 h-1) P^{\prime \prime \prime}=(-5 P-28 h+6) P^{\prime \prime}-\left(5 P^{\prime}+8\right) P^{\prime} .
\end{aligned}
$$

It follows from (6.11) that $\lim _{h \rightarrow 0} P^{\prime \prime}(h)>0$. Suppose that there exists an $h_{0}$ such that $P^{\prime \prime}\left(h_{0}\right)=0$ and $P^{\prime \prime}(h)>0$ when $0<h<h_{0}$. From (6.12) we get $P^{\prime \prime \prime}\left(h_{0}\right)>0$, which is a contradiction. Then $\left(I_{3} / I_{1}\right)^{\prime \prime}<0$ from $P^{\prime \prime}(h)>0$ and (6.9). Thus, $M(h, \alpha, \beta)$ has almost two roots on the interval $h \in(0,1 / 4)$. Furthermore, there exists an $\alpha^{*}<0$ such that system (6.5) has at most two limit cycles when $0<\beta<-\alpha^{*}$.

Secondly, we prove that there are at most two limit cycles of system (6.5) when $0<\beta<-\alpha$ for general $\alpha<0$. Assume that there are at least three limit cycles of system (6.5) when $0<\beta<-\alpha$. Then system (6.6) has at least three limit cycles. Denote the three innermost limit cycles of system (6.6) by $\gamma_{1}, \gamma_{2}$ and $\gamma_{3}$, from inside to outside. Since the origin of system (6.5) is unstable when $0<\beta<-\alpha$, so is the origin of system (6.6). Then $\gamma_{1}$ is internally stable. Without loss of generality, we suppose that $\gamma_{1}, \gamma_{2}$ and $\gamma_{3}$ are simple and stable, unstable and stable respectively. Otherwise, from small perturbations and the rotation of the vector field we can still get such limit cycles.

Let $\left(d_{i}, 0\right)$ be the intersection point of $\gamma_{i}$ and the $x$-axis, where $0<d_{i}<1$ and $i=1,2,3$. Since the vector filed of system (6.6) is $C^{1}$ and $\gamma_{1}, \gamma_{2}, \gamma_{3}$ are all simple, for sufficiently small $|\delta|$ or $|\epsilon|$ there are also at least three limit cycles when $(\alpha, \beta) \rightarrow(\alpha+\delta, \beta)$ or $(\alpha, \beta) \rightarrow(\alpha, \beta+\epsilon)$, in which the three innermost ones are stable, unstable and stable. Denote the intersection points of the positive $x$-axis and the three innermost limit cycles bifurcated from perturbation of $\alpha$ (resp. $\beta$ ) by $d_{1}^{\delta}, d_{2}^{\delta}$ and $d_{3}^{\delta}\left(\right.$ resp. $d_{2}^{\epsilon}, d_{2}^{\epsilon}$ and $d_{3}^{\epsilon}$ ), where $0<d_{i}^{\delta}, d_{i}^{\epsilon}<1$ and $i=1,2,3$. By [2, Lemma 3.2],

$$
d_{1}^{\delta}>d_{1}, \quad d_{2}^{\delta}<d_{2}, \quad d_{3}^{\delta}>d_{3}, \quad \text { when } \delta>0
$$

and

$$
d_{1}^{\epsilon}<d_{1}, \quad d_{2}^{\epsilon}>d_{2}, \quad d_{3}^{\epsilon}<d_{3}, \quad \text { when } \epsilon<0 .
$$

In other words, for system (6.6), the stable limit cycles expand and the unstable limit cycle compresses when $\alpha$ increases for a fixed $\beta$, the stable limit cycles compress and the unstable limit cycle expands when $\beta$ decreases for a fixed $\alpha$.

Fix $\beta$ and increase $\alpha<0$ until $\gamma_{1}$ and $\gamma_{2}$ overlap or $\gamma_{3}$ disappears by coinciding with the outer closed orbit. If the increase of $\alpha$ stops after it reaches $\alpha^{*}$, system (6.6) has at least three limit cycles for $\alpha=\alpha^{*}$, which conflicts with the proven fact. If the increase of $\alpha$ stops before it reaches $\alpha^{*}$, denote the stop point of $\alpha$ by $\alpha^{(1)}$ and the limit cycle generated by expansion or compression of $\gamma_{i}$ by $\gamma_{i}^{(1)}$. Moreover, denote the intersection point of $\gamma_{i}^{(1)}$ with the $x$-axis when $\alpha=\alpha^{(1)}$ by $\left(d_{i}^{(1)}, 0\right)$, where $0<d_{i}^{(1)} \leq 1$. Note that here $d_{1}^{(1)}$ may equal to $d_{2}^{(1)}$.

Fix $\alpha=\alpha^{(1)}$ and decrease $\beta>0$ until $\gamma_{1}^{(1)}$ disappears at the origin or $\gamma_{2}^{(1)}$ and $\gamma_{3}^{(1)}$ overlap. From [2, Lemma 3.1], system (6.5) has no small limit cycles when $\beta \leq 0$. So does system (6.6). Then the decrease of $\beta$ stops at a positive value, denoted by $\beta^{(2)}$. Let $\gamma_{i}^{(2)}$ be the limit cycle 
generated by expansion or compression of $\gamma_{i}^{(1)}$ and $\left(d_{i}^{(2)}, 0\right)$ be the intersection point of $\gamma_{i}^{(2)}$ with the $x$-axis when $\alpha=\alpha^{(1)}$ and $\beta=\beta^{(2)}$ where $0 \leq d_{i}^{(1)}<1$. Note that here $d_{1}^{(2)}=0$ or $d_{2}^{(2)}=d_{3}^{(2)}$.

Then repeat the next two steps from $j=1$ until $\alpha \geq \alpha^{*}$

(a) Fix $\beta=\beta^{(2 j)}$ and increase $\alpha<0$ from $\alpha^{(2 j-1)}$ to get the disappearing statement of one of $\gamma_{1}^{(2 j)}, \gamma_{2}^{(2 j)}, \gamma_{3}^{(2 j)}$. Update $\alpha^{(2 j-1)}, \gamma_{i}^{(2 j)}$ and $d_{i}^{(2 j)}$ to $\alpha^{(2 j+1)}, \gamma_{i}^{(2 j+1)}$ and $d_{i}^{(2 j+1)}$.

(b) Fix $\alpha=\alpha^{(2 j+1)}$ and decrease $\beta>0$ from $\beta^{(2 j)}$ to get the disappearing statement of one of $\gamma_{1}^{(2 j+1)}, \gamma_{2}^{(2 j+1)}, \gamma_{3}^{(2 j+1)}$. Update $\beta^{(2 j)}, \gamma_{i}^{(2 j+1)}$ and $d_{i}^{(2 j+1)}$ to $\beta^{(2 j+2)}, \gamma_{i}^{(2 j+2)}$ and $d_{i}^{(2 j+2)}$.

We claim that this cyclic process will stop in finite steps. In fact, from the proof of $[2$, Lemma 3.2] the solutions of system (6.6) depend on $\alpha$ and $\beta$ in the same degree. If $n$ goes to infinity, we have $\lim _{n \rightarrow \infty}\left(d_{1}^{(n)}-d_{3}^{(n)}\right) \neq 0$, implying $\lim _{n \rightarrow \infty}\left(\alpha^{(2 n+1)}-\alpha^{(2 n-1)}\right) \neq 0$. Thus, after finite steps the increase of $\alpha$ stops after it reaches $\alpha^{*}$, Therefore, system (6.6) has at least three limit cycles for $\alpha=\alpha^{*}$, which is a contradiction. Furthermore, when $0<\beta<-\alpha$ system (6.5) has at most two limit cycles for general $\alpha<0$.

Finally, assume that there are two limit cycles for system (6.5). If only one of two limit cycles is semi-stable, by [2, Lemma 3.2] and [21, Theorem 3.4 of Chapter 3.4], at least two limit cycles will be bifurcated from the semi-stable one and the other one will not disappear. If both two limit cycles are semi-stable, since they are adjacent to each other, at least four limit cycles bifurcated from them. Then one limit cycle is stable and the other one is unstable. By [2, Lemma 2.1], the origin is unstable when $\alpha<0$. Thus the inner one is internally stable, indicating the inner limit cycle is stable and the outer one is unstable. Moreover, if there is a unique small limit cycle, we can only obtain it is internally stable, which completes the proof.

Remark that the methods of the proof in Lemma 6.1 can also be used to prove [6, Proposition 5.3] in the focus case of a Rayleigh-Duffing oscillator, which shows that at most two limit cycles surround the equilibrium $E_{R}$ and a double limit cycle bifurcation happens.

By Lemma 6.1, we can correct [2, Proposition $3.1(\mathrm{~d})$ ], and obtain double limit cycle bifurcations when $\varphi(\alpha)<\beta<-\alpha$.

Lemma 6.2. There exists a decreasing $C^{0}$ function $\psi(\alpha)$ for $\alpha<0$ such that $\varphi(\alpha)<\psi(\alpha)<-\alpha$ and

(a) when $\beta=\varphi(\alpha)$, system (6.5) exhibits a unique limit cycle, which is stable;

(b) when $\beta=\psi(\alpha)$, system (6.5) exhibits a unique limit cycle, which is internally stable, externally unstable;

(c) when $\varphi(\alpha)<\beta<\psi(\alpha)$, system (6.5) exhibits two limit cycles. The inner one is stable and the outer one is unstable;

(d) when $\beta>\psi(\alpha)$, system (6.5) exhibits no limit cycles. 
Proof. When $0<\beta=\varphi(\alpha)$ and $\alpha<0$, the existence of limit cycles comes from the internal instability of the 2-saddle loop and instability of the origin. By Lemma 6.1, there is a unique limit cycle coexisting with the 2-saddle loop. The statement (a) is proven. By [2, Lemma 3.2] and [21, Theorem 3.4 of Chapter 3.4], there exists a $\epsilon>0$ such that system (6.6) exhibits two limit cycles when $\beta=\varphi(\alpha)+\epsilon$, where the inner one is stable and the outer one is unstable. Moreover, the stable limit cycles expand and the unstable limit cycle compresses when $\beta$ increases for a fixed $\alpha$. Due to [2, Lemma 3.3], there are no limit cycles for system (6.6) when $\beta=-\alpha$. Then there exists a function $\psi(\alpha)$ for $\alpha<0$ such that the stable limit cycles and the unstable limit cycle overlap if and only if $\beta=\psi(\alpha)$. The statements (b)-(d) are proven.

For any $\alpha^{*}<0$ and sufficiently small $\epsilon>0$, system (6.5) exhibits two limit cycles when $\alpha=\alpha^{*}-\epsilon$ and $\beta=\psi\left(\alpha^{*}\right)$ by [2, Lemma 3.2] and Lemma 6.1. Moreover, the inner limit cycle is stable and the outer one is unstable. To keep the existence of the semi-stable limit cycle, we need to increase $\beta$ until the two limit cycles coincide. Then $\psi\left(\alpha^{*}-\epsilon\right)>\psi\left(\alpha^{*}\right)$, which means that $\psi(\alpha)$ is decreasing. Moreover, from the proof of [2, Lemma 3.2] the ordinates of the intersections of limit cycles with $y$-axis continuously depend on $\alpha$ and $\beta$, which implies the continuity of $\psi(\alpha)$. It completes the proof.

Hence, there is another global bifurcation curve in the global bifurcation diagram in [2, Fig.1], which is the double small limit cycle bifurcation curve $D L:=\left\{(\alpha, \beta) \in \mathbb{R}^{2}: \alpha<0, \beta=\psi(\alpha)\right\}$, where $0<\varphi(\alpha)<\psi(\alpha)<-\alpha$. Furthermore, a new region $\mathrm{V}$ is separated from the region II in [2, Theorem 1.1] while the rest part is still retained and recollected as II, where

$$
\begin{aligned}
& \mathrm{II}:=\left\{(\alpha, \beta) \in \mathbb{R}^{2}: \alpha<0, \beta>\psi(\alpha)\right\}, \\
& \mathrm{V}:=\left\{(\alpha, \beta) \in \mathbb{R}^{2}: \alpha<0, \varphi(\alpha)<\beta<\psi(\alpha)\right\},
\end{aligned}
$$

as shown in Fig. 21.

In addition, numerical simulations in [2, Fig.12 (d)] and [2, Fig.13 (b)] show that system (6.5) exhibits a unique limit cycle near the saddles. That implies $D L$ and $H L$ are very close to each other.

Similar mistakes have been made in the study of a nonsmooth Rayleigh-Duffing oscillator [20]. For the saddle case of

$$
\dot{x}=y, \quad \dot{y}=\mu_{1} x+x^{3}-\left(\mu_{2}+|y|\right) y,
$$

where $\mu_{1}, \mu_{2}$ are real, the closed orbits in [20, Lemma 3.5] will not be unique. Under the condition of [20, Lemma 3.5] the origin is unstable and the 2-saddle loop is internally unstable if it exists. Then system (6.13) exhibits at least two limit cycles for some parameters. Unlike smooth system (6.5), Abelian integrals can not be used to prove that there are at most two limit cycles for nonsmooth system (6.13) because it is hard to determine the sign of the derivative of the ratio of an elliptic integral and an elementary function. Efforts are also made to rewrite system (6.13) as a piecewise Liénard system

$$
\ddot{x}+f(x, \operatorname{sgn}(y)) \dot{x}+g(x, \operatorname{sgn}(y))=0
$$




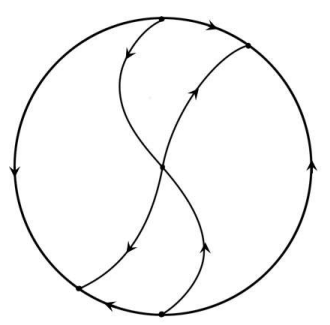

$\mathrm{I} \cup P$

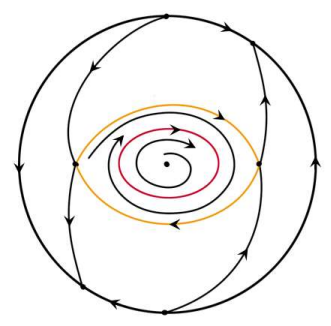

$H L$

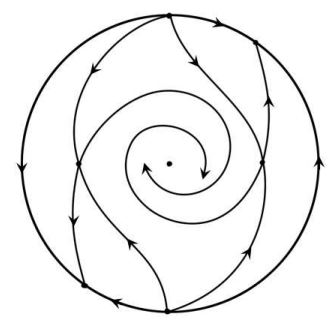

$\mathrm{IV} \cup H$

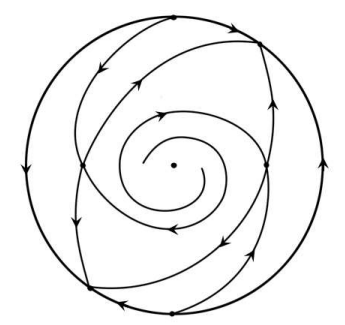

II

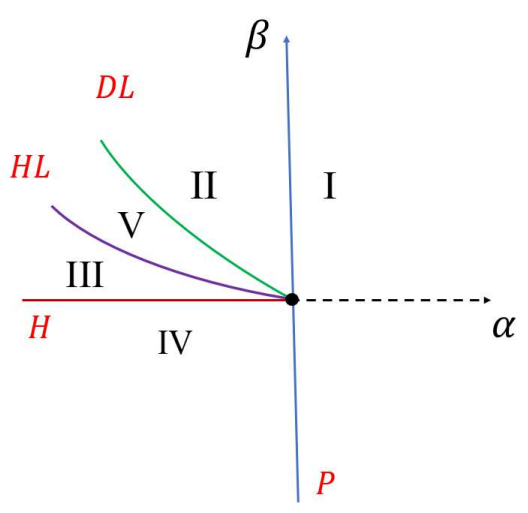

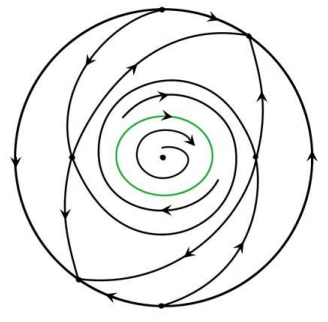

$D L$

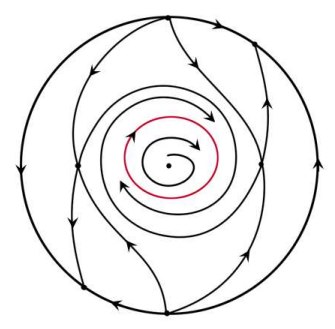

III

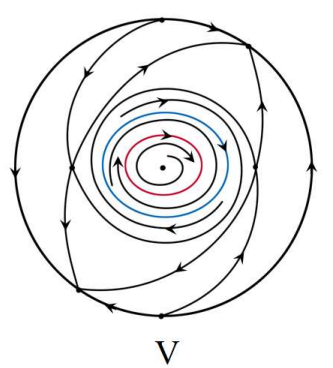

Fig. 21: The bifurcation diagram and revised global phase portraits of system (6.5).

and study the number of limit cycles. Although this method can be applied to smooth system (6.5), it also fails to nonsmooth system (6.13). That is because many mature theories about the number of limit cycles in Liénard system can not be used when $f$ is not an even function of $x$.

However, we can prove that there is a region where only stable limit cycles may exist.

Lemma 6.3. When $\mu_{1}<0$ and $-\left(-4 \mu_{1} / 3\right)^{3 / 4}<\mu_{2}<0$, system (1.2b) exhibits at most one limit cycle in the region $\left\{(x, y):|x| \leq \sqrt{-\mu_{1} / 3}\right\}$. Moreover, the limit cycle is hyperbolic and stable if it exists.

Proof. Assume that there is a limit cycle $\Gamma$ in the region $\left\{(x, y):|x| \leq \sqrt{-\mu_{1} / 3}\right\}$ for system (6.13). $\Gamma$ will cross the $x$-axis, the lines $y=-\mu_{2} / 2$ and $y=-\mu_{2}$ successively. Denote the intersections points by $A, B, C, D, E$ and $F$, as shown in Fig. 22. We will prove the uniqueness, hyperbolicity and stability of $\Gamma$ by showing $\oint_{\Gamma} \operatorname{div}\left(y, \mu_{1} x+x^{3}-\left(\mu_{2}+|y|\right) y\right) d t=2 \int_{\widehat{A F}}\left(-\mu_{2}-2 y\right) d t<0$.

The arc $\widehat{A B}$ can be regarded as the graph of the function $x=x_{1}(y), 0 \leq y \leq-\mu_{2} / 2$, and the arc $\widehat{B C}$ can be seen as the graph of the function $x=x_{2}(y)$ for $-\mu_{2} / 2 \leq y \leq-\mu_{2}$. Clearly, 


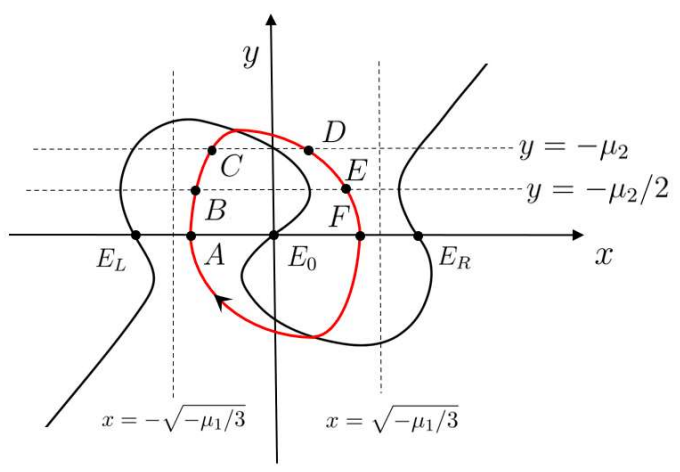

Fig. 22: A limit cycle in the region $\left\{(x, y):|x| \leq \sqrt{-\mu_{1} / 3}\right\}$.

$x_{1}\left(-\mu_{2} / 2\right)=x_{2}\left(-\mu_{2} / 2\right)$. Then

$$
\begin{aligned}
& \int_{\widehat{A C}}\left(-\mu_{2}-2 y\right) d t \\
& \quad=\int_{0}^{-\mu_{2} / 2} \frac{-\mu_{2}-2 y}{\mu_{1} x_{1}(y)+\left(x_{1}(y)\right)^{3}-\left(\mu_{2}+y\right) y} d y+\int_{-\mu_{2} / 2}^{-\mu_{2}} \frac{-\mu_{2}-2 y}{\mu_{1} x_{2}(y)+\left(x_{2}(y)\right)^{3}-\left(\mu_{2}+y\right) y} d y \\
& \quad=\int_{0}^{-\mu_{2} / 2} \frac{\left(-\mu_{2}-2 y\right)\left(x_{2}\left(-\mu_{2}-y\right)-x_{1}(y)\right)\left(\mu_{1}-\left(x_{1}(y)\right)^{2}-\left(x_{2}\left(-\mu_{2}-y\right)\right)^{2}-x_{1}(y) x_{2}\left(-\mu_{2}-y\right)\right)}{\left(\mu_{1} x_{1}(y)+\left(x_{1}(y)\right)^{3}-\left(\mu_{2}+y\right) y\right)\left(\mu_{1} x_{2}\left(-\mu_{2}-y\right)+\left(x_{2}\left(-\mu_{2}-y\right)\right)^{3}-\left(\mu_{2}+y\right) y\right)} d y \\
& \quad<0,
\end{aligned}
$$

because $x_{2}\left(-\mu_{2}-y\right)>x_{1}(y)$ for $0 \leq y \leq-\mu_{2} / 2$ and $|x| \leq \sqrt{-\mu_{1} / 3}$. Similarly,

$$
\int_{\widehat{D F}}\left(-\mu_{2}-2 y\right) d t<0
$$

Since $-\mu_{2}-2 y<0$ on the arc $\widehat{C D}$,

$$
\int_{\widehat{C D}}\left(-\mu_{2}-2 y\right) d t<0
$$

Thus,

$$
\oint_{\Gamma} \operatorname{div}\left(y, \mu_{1} x-x^{3}-\left(\mu_{2}+|y|\right) y\right) d t=2 \int_{\widehat{A F}}\left(-\mu_{2}-2 y\right) d t<0
$$

Outside the region $\left\{(x, y):|x| \leq \sqrt{-\mu_{1} / 3}\right\}$, at least two limit cycles may appear. So we correct [20, Proposition 3.1 (b), (c)] here.

Proposition 6.1. There exists a strictly increasing $C^{\infty}$ function $\varphi\left(\mu_{1}\right)$ for $\mu_{1}<0$ such that $-\left(-4 \mu_{1} / 3\right)^{3 / 4}<\varphi\left(\mu_{1}\right)<0$ and

(a) system (6.13) has one 2-saddle loop if and only if $\mu_{2}=\varphi\left(\mu_{1}\right)$;

(b) system (6.13) has at least one limit cycle when $\varphi\left(\mu_{1}\right) \leq \mu_{2}<0$;

(c) system (6.13) has at least two limit cycles when $\mu_{2}=\varphi\left(\mu_{1}\right)-\varepsilon$, where $\varepsilon>0$ is sufficiently small. 
Proof. The conclusion (a) is exact the same as [20, Proposition 3.1 (a)], which comes from the rotation of vector field and is not affected by the number of limit cycles. Moreover, the 2-saddle loop is internally unstable since the $\operatorname{tr} J_{R}=\operatorname{tr} J_{L}=-\mu_{2}>0$ when $\mu_{2}=\varphi\left(\mu_{1}\right)$, where Jacobian matrices $J_{R}$ and $J_{L}$ are defined in [20, Equation (2.3)]. Since the origin of system (6.13) is unstable by [20, Lemma 2.2], at least one limit cycle exists. Denote the outmost limit cycle by $\Gamma$. Clearly, $\Gamma$ is externally stable. When $\mu_{2}>\varphi\left(\mu_{1}\right)$, the existence of limit cycles can be obtained by the same methods in [20, Proposition 3.1 (b)]. When $\mu_{2}=\varphi\left(\mu_{1}\right)-\varepsilon$, by [20, Lemma 3.2], at least one limit cycles will be bifurcated from the 2-saddle loop, which is unstable and at least one limit cycles will be bifurcated from $\Gamma$, which is stable. Then the conclusion (c) is proven.

From the conclusion of numerical simulations, we cannot distinguish them even if there are only two limit cycles for system (6.13). Due to the results in smooth system (6.5), assume that there are at most two limit cycles for system (6.13). Then there is also a double limit cycle curve in global bifurcation diagram in [20, Fig.1], which is $D L:=\left\{\left(\mu_{1}, \mu_{2}\right) \in \mathbb{R}^{2}: \mu_{1}<0, \mu_{2}=\psi\left(\mu_{1}\right)\right\}$, where $0<-\left(-4 \mu_{1} / 3\right)^{3 / 4}<\psi\left(\mu_{1}\right)<\varphi\left(\mu_{1}\right)$.

Furthermore, a new region V is separated from the region IV in [20, Theorem 1.1] while the rest part is still retained and recollected as IV, where

$$
\begin{aligned}
\mathrm{IV} & :=\left\{\left(\mu_{1}, \mu_{2}\right) \in \mathbb{R}^{2}: \mu_{1}<0, \mu_{2}<\psi\left(\mu_{1}\right)\right\} \\
\mathrm{V} & :=\left\{\left(\mu_{1}, \mu_{2}\right) \in \mathbb{R}^{2}: \mu_{1}<0, \psi\left(\mu_{1}\right)<\mu_{2}<\varphi\left(\mu_{1}\right)\right\}
\end{aligned}
$$

as shown in Fig. 23.

Numerical simulations in [2, Fig.16 (b)] and [2, Fig. 18 (b)] show that system (6.13) exhibits a unique limit cycle near the saddles, which provide evidence that $D L$ and $H L$ are very close to each other.

\section{Data Availability Statement}

All data, models, and code generated or used during the study appear in the submitted article.

\section{Acknowledgements}

The first author is supported by the Applied Fundamental Research Program of Sichuan Province (No. 2020YJ0264). The second author is supported by the National Natural Science Foundation of China (No. 11801079). The third author is supported by the National Natural Science Foundations of China (Nos. 11931016, 11871041), the International Cooperation Fund of Ministry of Science and Technology of China and Science and Technology Innovation Action Program of STCSM (No. 20JC1413200). 


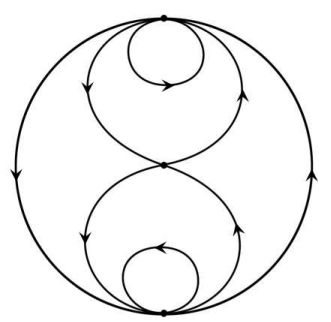

$\mathrm{I} \cup P$

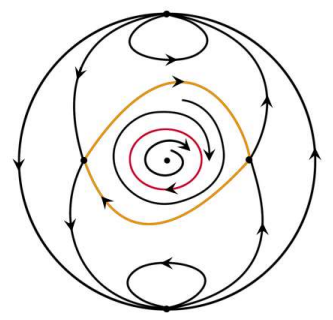

$H L$

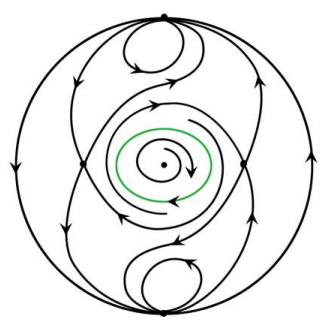

$D L$

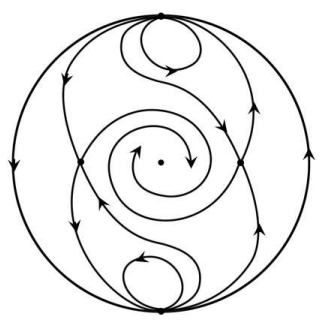

II $\cup H$

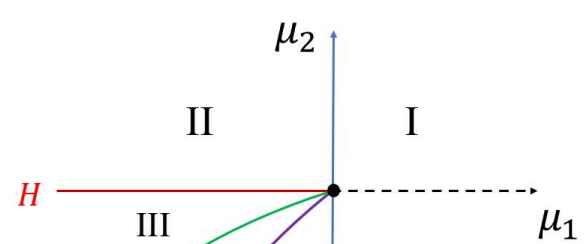

$\mu_{1}$

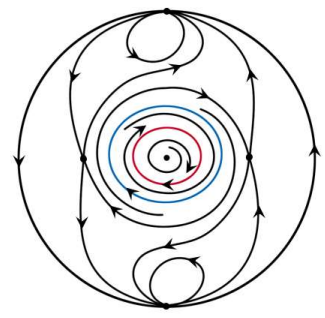

$\mathrm{V}$

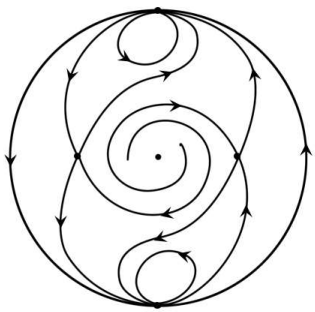

IV

Fig. 23: The bifurcation diagram and revised global phase portraits of system (6.13).

\section{Conflict of interest}

The authors declare that they have no conflict of interest.

\section{References}

[1] M. Bikdash, B. Balachandran, A. H. Nayfeh, Melnikov analysis for a ship with a general Roll-damping model, Nonlinear Dyn. 6 (1994), 101-124.

[2] H. Chen, D. Huang, Y. Jian, The saddle case of Rayleigh-Duffing oscillators, Nonlinear Dyna. 93 (2018), 22832300 .

[3] H. Chen, X. Chen, Global phase portraits of a degenerate Bogdanov-Takens system with symmetry (II), Discrete Contin. Dyn. Syst. Ser. B 23 (2018), 4141-4170.

[4] H. Chen, S. Duan, Y. Tang, J. Xie, Global dynamics of a mechanical system with dry friction, J. Differential Equations 265 (2018), 5490-5519.

[5] H. Chen, Y. Tang, An oscillator with two discontinuous lines and Van der Pol damping, Bull. Sci. math. 161 (2020), 102867

[6] H. Chen, L. Zou, Global study of Rayleigh-Duffing oscillators, J. Phys. A 49 (2016), 165202.

[7] S. N. Chow, C. Li, D. Wang, Normal Forms and Bifurcation of Planar Vector Fields, Cambridge Univ. Press, New York, 1994.

[8] J. Guckenheimer, P. Holmes, Nonlinear Oscillations, Dynamical Systems, and Bifurcations of Vector Fields, Springer-Verlag, New York, 1983. 
[9] P. Holmes and D. A. Rand, Phase portraits and bifurcations of the nonlinear oscillator $\ddot{x}+\left(\alpha+\gamma x^{2}\right) \dot{x}+\beta x+\delta x^{3}=$ 0, Int. J. Non-linear Mech. 15 (1980), 449-458.

[10] T. Küpper, H. D. Mittelmann, H. Weber, Numerical Methods for Bifurcation Problems, Birkhäuser Basel, 1984.

[11] N. Levinson, O. K. Smith, A general equation for relaxation oscillations, Duke Math. J. 9 (1942), 382 -403.

[12] A. Lins, W. de Melo, C. C. Pugh, On Liénard's equation, Lecture Notes in Math. 597 (1977), 335-357.

[13] A. H. Nayfeh, B. Balachandran, Applied Nonlinear Dynamics, Wiley, Weinheim, 2004.

[14] L.M. Perko, Differential Equations and Dynamical Systems, Springer, New York, 2002.

[15] L. Rayleigh, The Theory of Sound, Dover, New York, 1945.

[16] H. Troger, A. Steindl, Nonlinear Stability and Bifurcation Theory, Springer, Wien, 1991.

[17] Y. Tang, W. Zhang, Generalized normal sectors and orbits in exceptional directions, Nonlinearity 17 (2004), 1407-1426.

[18] B. Van der Pol, The nonlinear theory of electric oscillations, Proc. IRE 22 (1934), 1051-1085.

[19] Z. Wang, H. Chen, Nonsmooth van der Pol-Duffing oscillators: (I) The sum of indices of equilibria is -1 , Discrete Contin. Dyn. Syst. Ser. B, to appear.

[20] Z. Wang, H. Chen, The saddle case of a nonsmooth Rayleigh-Duffing oscillator, Int. J. Non-Linear Mechanics 129 (2021), 103657-1-16.

[21] Z. Zhang, T. Ding, W. Huang, Z. Dong, Qualitative Theory of Differential Equations, Transl. Math. Monogr. 101, Amer. Math. Soc., Providence, RI, 1992. 